TRANSACTIONS OF THE

AMERICAN MATHEMATICAL SOCIETY

Volume 358 , Number 6 , Pages $2447-2488$

S 0002-9947(06)04072-4

Article electronically published on January 24, 2006

\title{
GLOBAL WELL-POSEDNESS IN THE ENERGY SPACE FOR A MODIFIED KP II EQUATION VIA THE MIURA TRANSFORM
}

\author{
CARLOS E. KENIG AND YVAN MARTEL
}

\begin{abstract}
We prove global well-posedness of the initial value problem for a modified Kadomtsev-Petviashvili II (mKP II) equation in the energy space. The proof proceeds in three main steps and involves several different techniques.

In the first step, we make use of several linear estimates to solve a fourthorder parabolic regularization of the mKP II equation by a fixed point argument, for regular initial data (one estimate is similar to the sharp Kato smoothing effect proved for the KdV equation by Kenig, Ponce, and Vega, 1991).

Then, compactness arguments (the energy method performed through the Miura transform) give the existence of a local solution of the mKP II equation for regular data.

Finally, we approximate any data in the energy space by a sequence of smooth initial data. Using Bourgain's result concerning the global well-posedness of the KP II equation in $L^{2}$ and the Miura transformation, we obtain convergence of the sequence of smooth solutions to a solution of mKP II in the energy space.
\end{abstract}

\section{INTRODUCTION}

This paper is concerned with the initial value problem (IVP) for a modified Kadomtsev-Petviashvili II (mKP II) equation

$$
\partial_{t} u+\partial_{x}^{3} u+3 \partial_{x}^{-1} \partial_{y}^{2} u-6 u^{2} \partial_{x} u+6 \partial_{x} u \partial_{x}^{-1} \partial_{y} u=0 .
$$

(We explain below what we mean by $\partial_{x}^{-1}$.) A first motivation for studying this equation is its relation with the Kadomtsev-Petviashvili II (KP II) equation

$$
\partial_{t} v+\partial_{x}^{3} v+3 \partial_{x}^{-1} \partial_{y}^{2} v+6 v \partial_{x} v=0
$$

through the Miura transform:

$$
\text { If } u \text { satisfies (1) and } v=\partial_{x} u+\partial_{x}^{-1} \partial_{y} u-u^{2} \text {, then } v \text { satisfies (2). }
$$

Received by the editors April 22, 2004.

2000 Mathematics Subject Classification. Primary 35Q53; Secondary 35G25.

Key words and phrases. Modified KP II equation, initial value problem, Miura transform, energy method.

The first author was supported in part by the NSF and at IAS by The von Neumann Fund, The Oswald Veblen Fund and the Bell Companies Fellowship.

Part of this work was done while the second author was a Member of the IAS. He was partially supported by the NSF, under agreement No. DMS-0111298.

(C)2006 American Mathematical Society 
This observation was first made by Konopelchenko [14] (for the reader's convenience, (31) is proved in Appendix A). Transformation (3) is a generalization of the well-known Miura transform $v=\partial_{x} u-u^{2}$, connecting solutions of the modified Korteweg-de Vries $(\mathrm{mKdV})$ equation

$$
\partial_{t} u+\partial_{x}^{3} u-6 u^{2} \partial_{x} u=0
$$

to solutions of the $\mathrm{KdV}$ equation

$$
\partial_{t} v+\partial_{x}^{3} v+6 v \partial_{x} v=0
$$

(see Miura [20]).

The mKP II equation is the first equation in the modified KP hierarchy; we refer the reader to Jimbo and Miura 7] and references therein for the determination of the KP hierarchies. Several motivations for considering the mKP II equation are discussed in Konopelchenko and Dubrovsky [16]: apart from purely algebraic interests, like the connection to the KP II equation through the Miura transform, or the development of an Inverse Scattering Transform method for the mKP II equation, the mKP II equation may appear in the study of water waves in situations where a cubic nonlinearity is relevant (as the $\mathrm{mKdV}$ equation). In the present paper, using the mKP II equation, we obtain a new qualitative result on some solutions of the KP II equation (see Corollary 3).

The Miura transformation in the context of the KP II equations has already been studied in the Inverse Scattering Transform literature: see Wickerhauser [26] for invertibility properties of an operator related to (3), and well-posedness results for the KP II hierarchy (and in particular for (10) for small and regular data (Theorem V.3 in [26]). See also Konopelchenko and Dubrovsky [15], [16] for a study of exact solutions of (2) and (11).

Recall that the following quantities are formally conserved for solutions of (2):

$$
\begin{aligned}
& \int v(t)=\int v(0), \quad \int v^{2}(t)=\int v^{2}(0), \\
& \int\left\{\left(\partial_{x} v(t)\right)^{2}-3\left(\partial_{x}^{-1} \partial_{y} v(t)\right)^{2}-v^{3}(t)\right\} \\
& \quad=\int\left\{\left(\partial_{x} v(0)\right)^{2}-3\left(\partial_{x}^{-1} \partial_{y} v(0)\right)^{2}-v^{3}(0)\right\} .
\end{aligned}
$$

There are in fact an infinite number of conserved quantities for the KP II equation. Similarly, the following quantities are conserved through the mKP II flow:

$$
\begin{aligned}
& \int u^{2}(t)=\int u^{2}(0), \\
& \int\left\{\left(\partial_{x} u(t)\right)^{2}+\left(\partial_{x}^{-1} \partial_{y} u-u^{2}\right)^{2}\right\}=E(u(t))=E(u(0)) .
\end{aligned}
$$

To formally see the first conservation, it suffices to multiply equation (11) by $u$ and integrate by parts. The conservation of energy follows for example from the conservation of $L^{2}$ norm for (2) and the Miura transform. (Indeed, formally we have $\int \partial_{x} u\left(\partial_{x}^{-1} \partial_{y} u\right)=-\int u \partial_{y} u=0$ and $\int\left(\partial_{x} u\right) u^{2}=0$.) Observe that the conservation of $E(u(t))$ and the Sobolev-type inequality (see [1])

$$
\|w\|_{L^{4}} \leq C\left\|\partial_{x} w\right\|_{L^{2}}^{\frac{1}{2}}\|w\|_{L^{2}}^{\frac{1}{4}}\left\|\partial_{x}^{-1} \partial_{y} w\right\|_{L^{2}}^{\frac{1}{4}}
$$


give uniform bounds on solutions in the energy space $\mathcal{E}$ defined by $\mathcal{E}=\left\{u \in L^{2}(\mathbf{R}),\|u\|_{\mathcal{E}}<\infty\right\}$, where $\|u\|_{\mathcal{E}}=\left\|\partial_{x} u\right\|_{L^{2}}+\|u\|_{L^{2}}+\left\|\partial_{x}^{-1} \partial_{y} u\right\|_{L^{2}}$. Let us now comment on the notation $\partial_{x}^{-1} \partial_{y} u$ : if $u \in L^{2}$ is such that $\frac{|\mu|}{|\xi|} \hat{u} \in L_{\xi, \mu}^{2}$, where $\hat{u}(\xi, \mu)$ is the Fourier transform of $u$ in the two variables $x, y$, then we define the $L^{2}$ function $\partial_{x}^{-1} \partial_{y} u$ by $\widehat{\partial_{x}^{-1} \partial_{y}} u=\frac{\mu}{\xi} \hat{u}$ (of course $\partial_{x}\left(\partial_{x}^{-1} \partial_{y} u\right.$ ) $=\partial_{y} u$ ), thus $\left(\partial_{x}^{-1} \partial_{y}\right)$ has to be understood as one single operator. Then, $\partial_{x}^{-1} \partial_{y}^{2} u$ can be defined in the sense of distributions (or as an element of $\left.H^{-1}\right)$ as $\partial_{y}\left(\partial_{x}^{-1} \partial_{y} u\right)$.

We briefly recall known results concerning the Cauchy problem for the KP II equation. They are quite satisfactory, since Bourgain 4 proved that the initial value problem

$$
\left\{\begin{array}{l}
\partial_{x}\left(\partial_{t} v+\partial_{x}^{3} v+6 v \partial_{x} v\right)+3 \partial_{y}^{2} v=0, \quad t \in \mathbf{R},(x, y) \in \mathbf{R}^{2}, \\
v(0, x, y)=v_{0}(x, y)
\end{array}\right.
$$

is globally well posed for initial data $v_{0} \in L^{2}\left(\mathbf{R}^{2}\right)$ (see references in 4 for previous results). With respect to (2), the formulation (9) avoids having to give a sense to $\partial_{x}^{-1} \partial_{y}^{2} v$. Bourgain's proof and his notion of solution requires the introduction of specific spaces $X^{s, b}$ and $X_{T}^{s, b} \subset C\left([0, T], L^{2}\right)$, in which a fixed point argument is performed and local well-posedness is obtained. In particular, uniqueness is known to hold in $X_{T}^{s, b}$. Global well-posedness is then due to the invariant $\int v^{2}(t)$. We refer to Section 4 for a definition of these spaces and a precise well-posedness result (see Theorem 77). Following Bourgain, several authors have studied local and global well-posedness for initial data in negative exponent Sobolev spaces; see for example Takaoka and Tzvetkov 24] and Isaza and Mejía [19].

If we consider natural generalizations of the KP II equation, for example

$$
\partial_{x}\left(\partial_{t} v+\partial_{x}^{3} v+v^{k} \partial_{x} v\right)+3 \partial_{y}^{2} v=0
$$

for $k \geq 1$, classical techniques can be applied to equation (10), for example parabolic regularization and energy method provide well-posedness in spaces included in $H^{s}\left(\mathbf{R}^{2}\right)$ for $s>2$ (see Ukai [25], Saut [23] and Iório and Nunes 6]). Further well-posedness results are also proved by Kenig and Ziesler [12, 13, using maximal function estimates. For the generalized $\mathrm{KdV}$ equations, the Cauchy problem has been solved by Kenig, Ponce and Vega [11] in Sobolev spaces.

We now turn to the initial value problem

$$
\left\{\begin{array}{l}
\partial_{t} u+\partial_{x}^{3} u+3 \partial_{x}^{-1} \partial_{y}^{2} u-6 u^{2} \partial_{x} u+6 \partial_{x} u \partial_{x}^{-1} \partial_{y} u=0, \quad t \in \mathbf{R},(x, y) \in \mathbf{R}^{2} \\
u(0, x, y)=u_{0}(x, y) .
\end{array}\right.
$$

To our knowledge, apart from Wickerhauser's result [26] for small and regular data, no general well-posedness theory has been developed for the IVP (11), even for regular data $u_{0}$. With respect to (10), the bilinear term $\partial_{x} u \partial_{x}^{-1} \partial_{y} u$ creates serious additional difficulties. For example, differentiating equation (11) with respect to $x$ does not get rid of the $\partial_{x}^{-1}$ in the equation, thus one really has to give a sense to the product $\partial_{x} u \partial_{x}^{-1} \partial_{y} u$. Another serious difficulty is that the energy method does not work directly for (11). We thus have chosen to solve this equation by using the Miura transform (3). Note that this transformation is not bijective, and so Bourgain's result on the KP II equation cannot be used directly to obtain solutions of (11).

Our main result is the following theorem. 
Theorem 1. For any $u_{0} \in \mathcal{E}$ there exists a solution $u \in C(\mathbf{R}, \mathcal{E}) \cap C^{1}\left(\mathbf{R}, H^{-2}(\mathbf{R})\right)$ of (11) such that the following are true:

(i) Miura transform: If $v \in C\left(\mathbf{R}, L^{2}\right)$ is defined by (3), then $v$ is the unique solution of (9) in the class $X^{0, b}, b>\frac{1}{2}$ close to $\frac{1}{2}$.

(ii) Persistence: If $\partial_{x} u_{0} \in H^{s}$ and $\partial_{x}^{-1} \partial_{y} u_{0} \in H^{s}$ for $s \geq 1$, then $u, \partial_{x} u \in$ $C\left(\mathbf{R}, H^{s}\right)$ and $\partial_{x}^{-1} \partial_{y} u \in C\left(\mathbf{R}, H^{s}\right)$.

(iii) Regularity: For any $T>0$, there exists a neighborhood $V$ of $u_{0}$ in $\mathcal{E}$ such that the map $\tilde{u}_{0} \mapsto \tilde{u}$ is Lipschitz from $V$ to $C([-T, T], \mathcal{E})$.

(iv) Uniqueness: If $\partial_{x} u_{0} \in H^{3}$ and $\partial_{x}^{-1} \partial_{y} u_{0} \in H^{3}$, then $u$ is the unique solution of (11) such that $\partial_{x} u \in C\left(\mathbf{R}, H^{3}\right)$ and $\partial_{x}^{-1} \partial_{y} u \in C\left(\mathbf{R}, H^{3}\right)$.

(v) Conserved quantities: For all $t \in \mathbf{R}$, (6) and (7) hold.

(vi) Qualitative behavior: For any $\beta>0$, the following holds:

$\lim _{t \rightarrow+\infty} \int_{x>\beta t}\left\{\left(\partial_{x} u(t, x, y)\right)^{2}+\left(\partial_{x}^{-1} \partial_{y} u(t, x, y)\right)^{2}+u^{2}(t, x, y)+u^{4}(t, x, y)\right\} d x d y=0$.

Remark 1. Comments about uniqueness. Note that if we define

$$
\tilde{v}=-\partial_{x} u+\partial_{x}^{-1} \partial_{y} u-u^{2},
$$

then $\tilde{v}$ is also a solution of (9) in $X^{0, b}$ (see Appendix A). It follows in particular that the solution $u$ constructed in Theorem 1 is the unique solution of (11) such that $v$ and $\tilde{v}$ belong to $X^{0, b}$. Indeed, let $u_{1}$ and $u_{2}$ be two solutions of (11) corresponding to the same initial data $u_{0}$, and assume that $v_{1}, v_{2}$, and $\tilde{v}_{1}, \tilde{v}_{2}$, the corresponding Miura transforms of $u_{1}, u_{2}$, respectively, belong to $X^{0, b}$. Then by uniqueness for (9) in the class $X^{0, b}$ (see Theorem [7), we have $v_{1}=v_{2}$ and $\tilde{v}_{1}=\tilde{v}_{2}$, so that $\partial_{x} u_{1}=\partial_{x} u_{2}=\frac{1}{2}\left(v_{1}-\tilde{v}_{1}\right)$ and so $u_{1}=u_{2}$.

From Theorem 1 (ii)-(iv), we can make another statement concerning uniqueness. Let $u_{0} \in \mathcal{E}$, and let $u$ be the solution given by Theorem 11. Let $\left(u_{0 n}\right)$ be a sequence such that $\partial_{x} u_{0 n} \in H^{3}, \partial_{x}^{-1} \partial_{y} u_{0 n} \in H^{3}$ and $u_{0 n} \rightarrow u_{0}$ in $\mathcal{E}$ as $n \rightarrow+\infty$, and for each $n$, let $u_{n}$ be the unique solution of (11) such that $\partial_{x} u_{n} \in C\left(\mathbf{R}, H^{3}\right)$ and $\partial_{x}^{-1} \partial_{y} u_{n} \in C\left(\mathbf{R}, H^{3}\right)$ given by Theorem 1(ii) and (iv). Then by Theorem 1(iii), for all $T>0, u_{n} \rightarrow u$ in $C([-T, T], \mathcal{E})$ as $n \rightarrow+\infty$. Thus, $u$ is the unique solution of (11) which can be obtained as the limit of regular solutions.

Remark 2. By scaling, we can solve not only the IVP (11) but some other equations with the same terms but different coefficients. Because we use the Miura transform, there is a certain rigidity and the choice of the parameters is not completely free. More precisely, we can solve the IVP for

$$
\partial_{t} \tilde{u}+a \partial_{x}^{3} \tilde{u}+b \partial_{x}^{-1} \partial_{y}^{2} \tilde{u}+c \tilde{u}^{2} \partial_{x} \tilde{u}+d \partial_{x} \tilde{u} \partial_{x}^{-1} \partial_{y} \tilde{u}=0
$$

for any $a, b, c, d \in \mathbf{R}$ such that $a c>0$ and $2 b c=d^{2}$. Indeed, let $u(t, x, y)$ be a solution of the IVP (11) and let $\tilde{u}(t, x, y)=\alpha u(a t, x, \beta y)$, where $\alpha=\sqrt{\frac{3 a}{8 c}}$, $\beta=\sqrt{\frac{3 a}{b}}$. Then $\tilde{u}$ is a solution of (13). If the relation between $a, b, c, d$ above is not satisfied, the IVP might be difficult to solve. However, by the arguments in section 2 , we know how to solve locally in time a fourth-order parabolic regularization of (13) for regular initial data, for any set of parameters. See Proposition 4.

Theorem 1(vi) means that any solution of (11) goes to zero in the region $x>\beta t$, for any $\beta$. This is due to the fact that (11) has a defocusing nonlinearity. However, the behavior of $u(t, x)$ for $x<0$ is not known. One may also ask whether a similar 
result holds for the KP II equation (9). It is known that there is no traveling wave solution of (9) (see de Bouard and Saut [3]) and more precisely that there is no nontrivial solution of (9) that remains uniformly localized in space and travel to the right in some sense (see Theorem 1 in de Bouard and Martel [2]).

In the case where $v_{0} \in L^{2}$ can be related to a function $u_{0} \in \mathcal{E}$ by the Miura transform (3), we obtain from Theorem 1 the convergence to 0 of $v(t)$ in $L^{2}$ in the region $x>\beta t$, for any $\beta>0$. It is then a natural question to ask for which $v_{0} \in L^{2}$ there exists $u_{0} \in \mathcal{E}$ such that (3) holds. This question is partially answered by Wickerhauser (26, Theorem 1.II).

Theorem 2 (Wickerhauser, [26]). There exists $\alpha_{0}>0$ such that the following is true. If $V \in L^{1}\left(\mathbf{R}^{2}\right) \cap L^{2}\left(\mathbf{R}^{2}\right)$ satisfies $\|V\|_{L^{2}}+\|V\|_{L^{1}} \leq \alpha_{0}$, then there exists $U \in \mathcal{E}$ such that

$$
\partial_{x} U+\partial_{x}^{-1} \partial_{y} U-U^{2}=V
$$

The proof of this result is essentially contained in [26], but for the reader's convenience we repeat the proof in Appendix B.

By Theorem 2 and Theorem 1 , we obtain the following result.

Corollary 3. Let $v_{0} \in L^{1}\left(\mathbf{R}^{2}\right) \cap L^{2}\left(\mathbf{R}^{2}\right)$ and let $v \in C\left(\mathbf{R}, L^{2}\right) \cap X^{0, b}$ be the global solution of (9). There exists $\alpha_{0}>0$ such that if $\left\|v_{0}\right\|_{L^{2}}+\left\|v_{0}\right\|_{L^{1}} \leq \alpha_{0}$, then for all $\beta>0$,

$$
\lim _{t \rightarrow+\infty} \int_{x>\beta t} v^{2}(t, x, y) d x d y=0 .
$$

We briefly sketch the proof of Theorem 1 First by a fixed point argument, we solve locally in time a fourth-order parabolic regularization of the IVP (11) by using the smoothing effect and maximal function estimates (see section 2). The arguments are reminiscent of the ones used for the generalized KdV equations by Kenig, Ponce and Vega [10, [11.

Then, in section 3, relating solutions of this regularized equation to solutions of a regularized version of the KP II equation by the Miura transform, we use an energy method to obtain solutions to the mKP II equation for smooth data.

Finally, in section 4, we recall precisely Bourgain's result concerning the KP II equation, and we use it through the Miura transform to prove existence of a solution for initial data in $\mathcal{E}$. We approximate the initial data by a sequence of smooth functions, then we pass to the limit by using an estimate on the difference of two solutions of the KP II equation coming from Bourgain's result.

In section 5 , we present the proof of (v). It is based on a monotonicity property of the KP II equation, first proved for the KdV equation (see for example Martel and Merle [18]) and then for the KP II equation (see de Bouard and Martel [2]).

Notation. In this paper, the space variable is in $\mathbf{R}^{2}$, so that $L^{2}$ means $L^{2}\left(\mathbf{R}^{2}\right)$ and, unless otherwise mentioned, $\int$ means integration over $\mathbf{R}^{2}$.

- $\hat{f}(\xi, \mu)=\mathcal{F}(f)(\xi, \mu)=\int e^{-i(x \xi+y \mu)} f(x, y) d x d y$ is the Fourier transform of $f$ in the space variable. For this choice of the Fourier transform, we have $\|\hat{f}\|_{L^{2}}=$ $\frac{1}{2 \pi}\|f\|_{L^{2}}$ and $\mathcal{F}(f g)=\frac{1}{(2 \pi)^{2}} \hat{f} \star \hat{g}$.

$-J^{s}=(1-\Delta)^{s / 2}, D^{s}=(-\Delta)^{s / 2}$.

- $H^{s}=\left\{u \in L^{2}:\left\|J^{s} u\right\|_{L^{2}}=\|u\|_{H^{s}}<+\infty\right\}, \quad H^{\infty}=\bigcap_{s \geq 0} H^{s}$.

- $[A, B]=A B-B A$, where $A, B$ are operators. In particular, $\left[J^{s}, f\right] g=$ $J^{s}(f g)-f J^{s} g$, where a function $f$ is viewed as a multiplication operator. 


\section{General Well-Posedness Result FOR A REGULARIZED MKP II EQUATION}

We consider the IVP associated to the following fourth-order parabolic regularization of the mKP II equation

$$
\left\{\begin{array}{l}
\partial_{t} u+\partial_{x}^{4} u+\partial_{y}^{4} u+\partial_{x}^{3} u+3 \partial_{x}^{-1} \partial_{y}^{2} u+a u^{2} \partial_{x} u+b \partial_{x} u \partial_{x}^{-1} \partial_{y} u=0, \quad t>0 \\
u(0, x, y)=u_{0}(x, y)
\end{array}\right.
$$

for $a, b \in \mathbf{R}$. We consider the spaces, for $k, l \geq 1$,

$$
\begin{aligned}
Y^{k, l} & =\left\{u \in L^{2}\left(\mathbf{R}^{2}\right) ; u \in H^{k}\left(\mathbf{R}^{2}\right), \partial_{x}^{-1} \partial_{y} u \in H^{l}\left(\mathbf{R}^{2}\right)\right\}, \\
Y=Y^{8,2} & =\left\{u \in L^{2}\left(\mathbf{R}^{2}\right) ; u \in H^{8}\left(\mathbf{R}^{2}\right), \partial_{x}^{-1} \partial_{y} u \in H^{2}\left(\mathbf{R}^{2}\right)\right\}, \\
Y^{\infty} & =\left\{u \in L^{2}\left(\mathbf{R}^{2}\right), u \in \bigcap_{k \geq 0} H^{k}\left(\mathbf{R}^{2}\right), \partial_{x}^{-1} \partial_{y} u \in \bigcap_{k \geq 0} H^{k}\left(\mathbf{R}^{2}\right)\right\},
\end{aligned}
$$

with natural associated norms for $Y$ and $Y^{k, l}$. Note that for $k \geq 1, Y^{k-1, k} \subset H^{k}$. Indeed, if $\partial_{x} u \in H^{k-1}, \partial_{y} u=\partial_{x}\left(\partial_{x}^{-1} \partial_{y} u\right) \in H^{k-1}$, and $u \in L^{2}$, then $u \in H^{k}$.

In this section, we prove the following local well-posedness result for the IVP (15) in $Y$, with persistence of regularity in $Y^{k, l}$. The result is proved by a contraction argument using suitable norms. In this result, no particular choice of $a, b \in \mathbf{R}$ is required. It is possible that the IVP (15) is also well posed with less regularity on $u_{0}$, but it was not our objective here to obtain a sharp result.

Proposition 4 (Local well-posedness for the regularized mKP II equation). For any $u_{0} \in Y$, there exists $T=T\left(a, b,\left\|u_{0}\right\|_{Y}\right)>0$ and a unique solution $u$ of (15) satisfying

$$
\begin{gathered}
u \in C([0, T], Y) \\
\left\|\partial_{x} u\right\|_{L_{x}^{2} L_{y T}^{\infty}}+\left\|\partial_{x}^{2} \partial_{x} u\right\|_{L_{x}^{2} L_{y T}^{\infty}}+\left\|\partial_{y}^{2} \partial_{x} u\right\|_{L_{x}^{2} L_{y T}^{\infty}}<\infty \\
\left\|\partial_{x}^{-1} \partial_{y} u\right\|_{L_{x}^{\infty} L_{y T}^{2}}+\left\|\partial_{x}^{8} \partial_{x}^{-1} \partial_{y} u\right\|_{L_{x}^{\infty} L_{y T}^{2}}+\left\|\partial_{y}^{8} \partial_{x}^{-1} \partial_{y} u\right\|_{L_{x}^{\infty} L_{y T}^{2}}<\infty \\
\sup _{[0, T]} t^{\frac{1}{4}}\left[\left\|\partial_{x} \partial_{x}^{8} u\right\|_{L_{x y}^{2}}+\left\|\partial_{y} \partial_{x}^{8} u\right\|_{L_{x y}^{2}}+\left\|\partial_{x} \partial_{y}^{8} u\right\|_{L_{x y}^{2}}+\left\|\partial_{y} \partial_{y}^{8} u\right\|_{L_{x y}^{2}}\right]<\infty .
\end{gathered}
$$

For any $T^{\prime} \in(0, T)$, there exists a neighborhood $V$ of $u_{0}$ in $Y$ such that the map $\tilde{u}_{0} \mapsto \tilde{u}(t)$ from $V$ into the class defined by (16)-(19) with $T^{\prime}$ instead of $T$ is Lipschitz.

Moreover, if $u_{0} \in Y^{\infty}$, then, for all $k, l \geq 0$,

$$
u \in C\left([0, T], Y^{k, l}\right)
$$

2.1. Linear estimates. We consider $S(t)$ the solution operator for the associated linear equation

$$
\left\{\begin{array}{l}
\partial_{t} w+\partial_{x}^{4} w+\partial_{y}^{4} w+\partial_{x}^{3} w+3 \partial_{x}^{-1} \partial_{y}^{2} w=0 \\
w(x, 0)=w_{0}(x)
\end{array}\right.
$$

Thus, for $t \geq 0$,

$$
S(t) w_{0}=c \iint e^{i\left[x \xi+y \mu+t\left(\xi^{3}-3 \frac{\mu^{2}}{\xi}\right)\right]-t \xi^{4}-t \mu^{4}} \hat{w}_{0}(\xi, \mu) d \xi d \mu .
$$


We extend $S(t)$ to $t<0$ by the formula

$$
S(t) w_{0}=c \iint e^{i\left[x \xi+y \mu+t\left(\xi^{3}-3 \frac{\mu^{2}}{\xi}\right)\right]-|t| \xi^{4}-|t| \mu^{4}} \hat{w}_{0}(\xi, \mu) d \xi d \mu .
$$

We begin by proving that $\partial_{x}^{-1} \partial_{y} S(t) w_{0}$ has a sense in $L_{x}^{\infty} L_{y t}^{2}$ for $w_{0}$ only in $L^{2}$. The proof is reminiscent from the proof of the sharp Kato smoothing effect for the Airy group (see Kenig, Ponce and Vega [10, Lemma 2.1).

Lemma 1. Let $w_{0} \in L^{2}\left(\mathbf{R}^{2}\right)$; then

$$
\left\|\partial_{x}^{-1} \partial_{y} S(t) w_{0}\right\|_{L_{x}^{\infty} L_{y t}^{2}} \leq C\left\|w_{0}\right\|_{L_{x y}^{2}} .
$$

Let $h \in L_{x}^{1} L_{y T}^{2}$ for $T>0$; then

$$
\sup _{0<t<T}\left\|\partial_{x}^{-1} \partial_{y} \int_{0}^{t} S\left(t-t^{\prime}\right) h\left(t^{\prime}\right) d t^{\prime}\right\|_{L_{x y}^{2}} \leq C\|h\|_{L_{x}^{1} L_{y T}^{2}} .
$$

Proof of (22). Fix $x \in \mathbf{R}$. Let us change the variables $\alpha=\mu, \beta=\xi^{3}-3 \frac{\mu^{2}}{\xi}$. We write $\xi=\xi(\alpha, \beta), \mu=\mu(\alpha, \beta)=\alpha$. Then

$$
S(t) w_{0}=\iint e^{i[x \xi+y \alpha+t \beta]-|t| \xi^{4}-|t| \mu^{4}} \hat{w}_{0}(\xi, \mu) \frac{d \alpha d \beta}{J(\alpha, \beta)},
$$

where $J(\alpha, \beta)$ is the Jacobian of the change of variable, i.e. $J=3\left(\xi+\frac{\mu^{2}}{\xi^{2}}\right)$. We have

$$
\begin{aligned}
\partial_{x}^{-1} \partial_{y} S(t) w_{0} & =\iint e^{i[x \xi+y \alpha+t \beta]-|t| \xi^{4}-|t| \mu^{4}} \frac{\mu}{\xi} \hat{w}_{0}(\xi, \mu) \frac{d \alpha d \beta}{J(\alpha, \beta)} \\
& =\iint e^{i[y \alpha+t \beta]-|t| \xi^{4}-|t| \mu^{4}} F(\alpha, \beta) d \alpha d \beta,
\end{aligned}
$$

where

$$
F(\alpha, \beta)=e^{i x \xi} \frac{\mu}{\xi} \frac{\hat{w}_{0}(\xi, \mu)}{J(\alpha, \beta)} .
$$

Let $m(t, \alpha, \beta)=e^{-|t| \xi^{4}-|t| \alpha^{4}}$. We claim the following estimate.

Claim 1. Let $F \in L_{\alpha \beta}\left(\mathbf{R}^{2}\right)$; then

$$
\left\|\int e^{i[y \alpha+t \beta]} m(t, \alpha, \beta) F(\alpha, \beta) d \alpha d \beta\right\|_{L_{y t}^{2}} \leq C\|F\|_{L_{\alpha \beta}^{2}} .
$$

Assuming Claim 1, we finish the proof of the lemma. By returning to the original variables $(\xi, \mu)$, we have

$$
\|F\|_{L_{\alpha, \beta}^{2}}^{2}=\iint\left|\frac{\mu}{\xi}\right|^{2} \frac{\left|\hat{w}_{0}(\xi, \mu)\right|^{2}}{J} \frac{d \alpha d \beta}{J}=\iint\left|\frac{\mu}{\xi}\right|^{2} \frac{\left|\hat{w}_{0}(\xi, \mu)\right|^{2}}{J} d \xi d \mu .
$$

Since $J(\xi, \mu)=3\left(\xi^{2}+\frac{\mu^{2}}{\xi^{2}}\right) \geq \frac{\mu^{2}}{\xi^{2}}$, the lemma follows.

Therefore we are reduced to prove Claim 1. By Plancherel's Theorem in the $y$ variable, and then Fubini's theorem,

$$
\begin{aligned}
\left\|\iint e^{i[y \alpha+t \beta]} m(t, \alpha, \beta) F(\alpha, \beta) d \alpha d \beta\right\|_{L_{y t}^{2}} & =\|\| \int e^{i t \beta} m(t, \alpha, \beta) F(\alpha, \beta) d \beta\left\|_{L_{\alpha}^{2}}\right\|_{L_{t}^{2}} \\
& =\|\| \int e^{i t \beta} m(t, \alpha, \beta) F(\alpha, \beta) d \beta\left\|_{L_{t}^{2}}\right\|_{L_{\alpha}^{2}} .
\end{aligned}
$$


Thus, we need only prove for $f \in L_{\beta}^{2}(\mathbf{R})$,

$$
\left\|\int e^{i t \beta} m(t, \alpha, \beta) f(\beta) d \beta\right\|_{L_{t}^{2}} \leq C\|f\|_{L_{\beta}^{2}},
$$

where $C$ is uniform in $\alpha$. If we show this, then we have

$$
\|\| \int e^{i t \beta} m(t, \alpha, \beta) F(\alpha, \beta) d \beta\left\|_{L_{t}^{2}}\right\|_{L_{\alpha}^{2}} \leq C\|\| F(\alpha, \beta)\left\|_{L_{\beta}^{2}}\right\|_{L_{\alpha}^{2}}=C\|F(\alpha, \beta)\|_{L_{\alpha \beta}^{2}} .
$$

To prove (24), it suffices to show that

$$
|m(t, \alpha, \beta)|+\int\left|\partial_{t} m(t, \alpha, \beta)\right| d t \leq C,
$$

where $C$ is uniform in $\alpha, \beta$. To see that (25) suffices, we use Carleson's Theorem in the following form: the operator

$$
C(h)(\beta)=\sup _{N \in \mathbf{R}}\left|\int_{-\infty}^{N} h(t) e^{-i t \beta} d t\right|
$$

is bounded from $L^{2}(d t)$ into $L^{2}(d \beta)$ (we refer to Coifman and Meyer [5], Chapter I, Lemma 12, for such a use of Carleson's theorem). Let $h \in L^{2}(d t)$. We need to estimate

$$
\iint e^{i t \beta} m(t, \alpha, \beta) f(\beta) h(t) d \beta d t=\int f(\beta)\left(\int e^{i t \beta} m(t, \alpha, \beta) h(t) d t\right) d \beta .
$$

But, by integration by parts,

$$
\begin{aligned}
\left|\int e^{i t \beta} m(t, \alpha, \beta) h(t) d t\right| & =\left|\int\left(\int_{-\infty}^{t} e^{i s \beta} h(s) d s\right)^{\prime} m(t, \alpha, \beta) d t\right| \\
& \leq|m(+\infty, \alpha, \beta)||\hat{h}(\beta)|+C(h)(\beta) \int\left|\partial_{t} m(t, \alpha, \beta)\right| d t
\end{aligned}
$$

and we see that Carleson's Theorem and (25) give the result. Now recall that $m(t, \alpha, \beta)=e^{-|t| \xi^{4}-|t| \alpha^{4}}$, where $\xi=\xi(\alpha, \beta)$, and so (25) follows.

Proof of (23). Fix $t^{*} \in(0, T)$, and change variable $t^{*}-t^{\prime}=t$ :

$$
\partial_{x}^{-1} \partial_{y} \int_{0}^{t^{*}} S\left(t^{*}-t^{\prime}\right) h\left(t^{\prime}\right) d t^{\prime}=\partial_{x}^{-1} \partial_{y} \int_{0}^{t^{*}} S(t) h_{t^{*}}(t) d t,
$$

where $h_{t^{*}}(x, y, t) \equiv h\left(x, y, t^{*}-t\right)$. To estimate the $L_{x y}^{2}$ norm, let us test against $w_{0} \in L_{x y}^{2}$. Then

$$
\left\langle\partial_{x}^{-1} \partial_{y} \int_{0}^{t^{*}} S(t) h_{t^{*}}(t) d t, w_{0}\right\rangle=\int_{0}^{t^{*}}\left\langle h_{t^{*}}(t), \partial_{x}^{-1} \partial_{y} S(t)^{*} w_{0}\right\rangle d t
$$

where $\langle$,$\rangle denotes the scalar product in L_{x y}^{2}$ and $S(t)^{*}$ is the adjoint operator of $S(t)$. Note that the proof of (22) above also gives

$$
\left\|\partial_{x}^{-1} \partial_{y} S(t)^{*} w_{0}\right\|_{L_{x}^{\infty} L_{y t}^{2}} \leq C\left\|w_{0}\right\|_{L_{x y}^{2}}
$$


Thus, for $\chi_{\left[0, t^{*}\right]}(t) \equiv 1$ on $\left[0, t^{*}\right]$ and $\chi_{\left[0, t^{*}\right]}(t) \equiv 0$ on $\mathbf{R} \backslash\left[0, t^{*}\right]$,

$$
\begin{aligned}
\left|\left\langle\partial_{x}^{-1} \partial_{y} \int_{0}^{t^{*}} S(t) h_{t^{*}}(t) d t, w_{0}\right\rangle\right| & =\left|\int\left\langle\chi_{\left[0, t^{*}\right]}(t) h_{t^{*}}(t), \partial_{x}^{-1} \partial_{y} S(t)^{*} w_{0}\right\rangle d t\right| \\
& \leq C\left\|w_{0}\right\|_{L_{x y}^{2}}\left\|\chi_{\left[0, t^{*}\right.} h_{t^{*}}\right\|_{L_{x}^{1} L_{y t}^{2}} \\
& \leq C\left\|w_{0}\right\|_{L_{x y}^{2}}\|h\|_{L_{x}^{1} L_{y T}^{2}},
\end{aligned}
$$

uniformly in $t^{*}$. This completes the proof of (23).

Lemma 2 (Maximal function estimate). Let $T>0$. Then,

$$
\begin{aligned}
& \left\|S(t) w_{0}\right\|_{L_{x}^{2} L_{y T}^{\infty}} \\
& \quad \leq C_{T}\left(\left\|w_{0}\right\|_{L_{x y}^{2}}+\left\|\partial_{x}^{5} w_{0}\right\|_{L_{x y}^{2}}\right. \\
& \left.\quad+\left\|\partial_{y}^{5} w_{0}\right\|_{L_{x y}^{2}}+\left\|\partial_{y} \partial_{x}^{-1} \partial_{y} w_{0}\right\|_{L_{x y}^{2}}+\left\|\partial_{y}^{2} \partial_{x}^{-1} \partial_{y} w_{0}\right\|_{L_{x y}^{2}}\right) .
\end{aligned}
$$

Proof. We first use the inequality

$$
\|f\|_{L_{y}^{\infty} L_{T}^{\infty}} \leq C_{T}\left\{\|f\|_{L_{y}^{2} L_{T}^{2}}+\left\|\partial_{y} f\right\|_{L_{y}^{2} L_{T}^{2}}+\left\|\partial_{t} f\right\|_{L_{y}^{2} L_{T}^{2}}+\left\|\partial_{t} \partial_{y} f\right\|_{L_{y}^{2} L_{T}^{2}}\right\} .
$$

We apply this to $f \equiv S(t) w_{0}$, so that

$$
\begin{aligned}
\left\|S(t) w_{0}\right\|_{L_{y}^{\infty} L_{T}^{\infty}} \leq & C_{T}\left\{\left\|S(t) w_{0}\right\|_{L_{y}^{2} L_{T}^{2}}+\left\|S(t) \partial_{y} w_{0}\right\|_{L_{y}^{2} L_{T}^{2}}\right. \\
& \left.+\left\|\partial_{t} S(t) w_{0}\right\|_{L_{y}^{2} L_{T}^{2}}+\left\|\partial_{t} S(t) \partial_{y} w_{0}\right\|_{L_{y}^{2} L_{T}^{2}}\right\} .
\end{aligned}
$$

We use the equation of $S(t) w_{0}$ to obtain, for fixed $x$,

$$
\begin{aligned}
& \left\|S(t) w_{0}\right\|_{L_{y}^{\infty} L_{T}^{\infty}} \leq C_{T}\left\{\left\|S(t) w_{0}\right\|_{L_{y}^{2} L_{T}^{2}}+\left\|S(t) \partial_{y} w_{0}\right\|_{L_{y}^{2} L_{T}^{2}}\right. \\
& \quad+\left\|\partial_{x}^{4} S(t) w_{0}\right\|_{L_{y T}^{2}}+\left\|\partial_{y}^{4} S(t) w_{0}\right\|_{L_{y T}^{2}}+\left\|\partial_{x}^{3} S(t) w_{0}\right\|_{L_{y T}^{2}}+\left\|\partial_{y} \partial_{x}^{-1} \partial_{y} S(t) w_{0}\right\|_{L_{y T}^{2}} \\
& \quad+\left\|\partial_{x}^{4} \partial_{y} S(t) w_{0}\right\|_{L_{y T}^{2}}+\left\|\partial_{y}^{4} \partial_{y} S(t) w_{0}\right\|_{L_{y T}^{2}}+\left\|\partial_{x}^{3} \partial_{y} S(t) w_{0}\right\|_{L_{y T}^{2}} \\
& \left.\quad+\left\|\partial_{y}^{2} \partial_{x}^{-1} \partial_{y} S(t) w_{0}\right\|_{L_{y T}^{2}}\right\} .
\end{aligned}
$$

Taking the $L^{2}$ norm in the $x$ variable and then $\sup _{[-T, T]}$, we get (27) by using $\|S(t) f\|_{L_{x y}^{2}} \leq\|f\|_{L_{x y}^{2}}$.

Lemma 3 (Smoothing estimate in $x, y$ ). Let $w_{0} \in L^{2}\left(\mathbf{R}^{2}\right)$. Then,

$$
\left\|\partial_{x} S(t) w_{0}\right\|_{L_{x y}^{2}}+\left\|\partial_{y} S(t) w_{0}\right\|_{L_{x y}^{2}} \leq \frac{C}{|t|^{\frac{1}{4}}}\left\|w_{0}\right\|_{L_{x y}^{2}} .
$$

Proof. We have

$$
\partial_{x} \widehat{S(t)} w_{0}(\xi, \mu)=c i \xi e^{-t|\xi|^{4}-t|\mu|^{4}} e^{i t\left(\xi^{3}-3 \frac{\mu^{2}}{\xi}\right)} \hat{w}_{0}(\mu, \xi),
$$

and thus the result follows immediately.

Finally, we present a lemma to be used to handle errors in Leibnitz rule (this result is due to Molinet and Ribaud 22]). Consider a function $\varphi \in C_{0}^{\infty}(\mathbf{R})$ such that $\varphi \equiv 1$ on $|\xi| \leq \frac{1}{2}, \operatorname{supp} \varphi \subset\{|\xi| \leq 1\}$. Let $\widehat{S_{k} f}=\varphi\left(\xi 2^{-k}\right) \hat{f}(\xi)$ and $\Delta_{k} f=$ $S_{k+1} f-S_{k} f$, for $k \geq 1$, and $\Delta_{0}(f)=S_{1}(f)$. 
Lemma 4. Let $f_{1}, f_{2} \in L^{2}$. Then, for $j \geq 3$, the following holds:

$$
\begin{aligned}
\Delta_{j}\left(f_{1} f_{2}\right)= & \Delta_{j}\left(\Delta_{0}\left(f_{1}\right) \Delta_{0}\left(f_{2}\right)\right)+\sum_{k \geq j-2} \Delta_{j}\left(\Delta_{k}\left(f_{1}\right) S_{k+1}\left(f_{2}\right)\right) \\
& +\sum_{k \geq j-2} \Delta_{j}\left(S_{k}\left(f_{1}\right) \Delta_{k}\left(f_{2}\right)\right) .
\end{aligned}
$$

Proof. We have

$$
\begin{aligned}
\Delta_{j}\left(f_{1} f_{2}\right)= & \Delta_{j}\left(\lim _{k \rightarrow+\infty} S_{k}\left(f_{1}\right) S_{k}\left(f_{2}\right)\right) \\
= & \Delta_{j}\left(\sum_{k=1}^{\infty} S_{k+1}\left(f_{1}\right) S_{k+1}\left(f_{2}\right)-S_{k}\left(f_{1}\right) S_{k}\left(f_{2}\right)\right) \\
& +\Delta_{j}\left(\Delta_{0}\left(f_{1}\right) \Delta_{0}\left(f_{2}\right)\right) \\
= & \Delta_{j}\left(\sum_{k=1}^{\infty}\left[S_{k+1}\left(f_{1}\right)-S_{k}\left(f_{1}\right)\right] S_{k+1}\left(f_{2}\right)\right) \\
& +\Delta_{j}\left(\sum_{k=1}^{\infty}\left[S_{k}\left(f_{1}\right)\left(S_{k+1}\left(f_{2}\right)-S_{k}\left(f_{2}\right)\right)\right]\right)+\Delta_{j}\left(\Delta_{0}\left(f_{1}\right) \Delta_{0}\left(f_{2}\right)\right) .
\end{aligned}
$$

Now, if $j \geq 3$, by support considerations on the Fourier transform side, we get

$$
\begin{aligned}
\Delta_{j}\left(f_{1} f_{2}\right)= & \Delta_{j}\left(\sum_{k=j-2}^{\infty} \Delta_{k}\left(f_{1}\right) S_{K+1}\left(f_{2}\right)\right)+\Delta_{j}\left(\sum_{k=j-2}^{\infty} S_{k}\left(f_{1}\right) \Delta\left(f_{2}\right)\right) \\
& +\Delta_{j}\left(\Delta_{0}\left(f_{1}\right) \Delta_{0}\left(f_{2}\right)\right) .
\end{aligned}
$$

Remark 3. A similar statement holds for $\Delta_{j}\left(f_{1} f_{2} f_{3}\right)$.

2.2. Proof of Proposition 4. Well-posedness in $Y$ via the fixed point Theorem. We define for $v$ on $[0, T] \times \mathbf{R}^{2}$ the following norms:

$$
\begin{aligned}
& \lambda_{1}(v)=\sup _{t \in[0, T]}\|v(t)\|_{Y}, \\
& \lambda_{2}(v)=\left\|\partial_{x} v\right\|_{L_{x}^{2} L_{y T}^{\infty}}+\left\|\partial_{x}^{2} \partial_{x} v\right\|_{L_{x}^{2} L_{y T}^{\infty}}+\left\|\partial_{y}^{2} \partial_{x} v\right\|_{L_{x}^{2} L_{y T}^{\infty},} \\
& \lambda_{3}(v)=\left\|\partial_{x}^{-1} \partial_{y} v\right\|_{L_{x}^{\infty} L_{y T}^{2}}+\left\|\partial_{x}^{8} \partial_{x}^{-1} \partial_{y} v\right\|_{L_{x}^{\infty} L_{y T}^{2}}+\left\|\partial_{y}^{8} \partial_{x}^{-1} \partial_{y} v\right\|_{L_{x}^{\infty} L_{y T}^{2} T}, \\
& \lambda_{4}(v)=\sup _{t \in[0, T]} t^{\frac{1}{4}}\left\{\left\|\partial_{x} \partial_{x}^{8} v\right\|_{L_{x y}^{2}}+\left\|\partial_{x} \partial_{y}^{8} v\right\|_{L_{x y}^{2}}+\left\|\partial_{y} \partial_{x}^{8} v\right\|_{L_{x y}^{2}}+\left\|\partial_{x} \partial_{y}^{8} v\right\|_{L_{x y}^{2}}\right\}, \\
& \Lambda_{T}(v)=\max _{j=1, \ldots, 4} \lambda_{j}(v) .
\end{aligned}
$$

Let

$$
\begin{aligned}
\Phi_{u_{0}}(v)= & S(t) u_{0}-a \int_{0}^{t} S\left(t-t^{\prime}\right) \partial_{x}\left(v^{3}\left(t^{\prime}\right)\right) d t^{\prime} \\
& -b \int_{0}^{t} S\left(t-t^{\prime}\right) \partial_{x} v\left(t^{\prime}\right)\left(\partial_{x}^{-1} \partial_{y} v\left(t^{\prime}\right)\right) d t^{\prime} \\
= & S(t) u_{0}-a \mathbf{I}(v)-b \mathbf{I I}(v) .
\end{aligned}
$$

We have the following result that implies immediately the existence and uniqueness result in $Y$. 
Claim 2. For any $u_{0} \in Y$, there exist $T=T\left(\left\|u_{0}\right\|_{Y}\right)>0$ and $\alpha=\alpha\left(\left\|u_{0}\right\|_{Y}\right)>0$ such that $\Phi_{u_{0}}: \mathcal{B}_{\alpha, T} \rightarrow \mathcal{B}_{\alpha, T}$ and is a contraction on $\mathcal{B}_{\alpha, T}$, where

$$
\mathcal{B}_{\alpha, T}=\left\{v \in C((0, T), Y) \text { s.t. } \Lambda_{T}(v)<\alpha\right\} .
$$

We prove Claim 2. First, note that $\lambda_{1}\left(S(t) u_{0}\right) \leq C\left\|u_{0}\right\|_{Y}$. Also, Lemma 3 shows that $\lambda_{4}\left(S(t) u_{0}\right) \leq C\left\|u_{0}\right\|_{Y}$, and Lemma 2 gives

$$
\begin{aligned}
\lambda_{2}\left(S(t) u_{0}\right) \leq & C_{T}\left\{\left\|\partial_{x} u_{0}\right\|_{L_{x y}^{2}}+\left\|\partial_{x}^{6} u_{0}\right\|_{L_{x y}^{2}}+\left\|\partial_{y}^{5} \partial_{x} u_{0}\right\|_{L_{x y}^{2}}+\left\|\partial_{y}^{2} u_{0}\right\|_{L_{x y}^{2}}\right. \\
& +\left\|\partial_{y}^{3} u_{0}\right\|_{L_{x y}^{2}}+\left\|\partial_{x}^{3} u_{0}\right\|_{L_{x y}^{2}}+\left\|\partial_{x}^{8} u_{0}\right\|_{L_{x y}^{2}}+\left\|\partial_{y}^{5} \partial_{x}^{3} u_{0}\right\|_{L_{x y}^{2}} \\
& +\left\|\partial_{y}^{2} \partial_{x}^{2} u_{0}\right\|_{L_{x y}^{2}}+\left\|\partial_{y}^{3} \partial_{x}^{2} u_{0}\right\|_{L_{x y}^{2}}+\left\|\partial_{y}^{2} u_{0}\right\|_{L_{x y}^{2}}+\left\|\partial_{y}^{2} \partial_{x}^{6} u_{0}\right\|_{L_{x y}^{2}} \\
& \left.+\left\|\partial_{y}^{7} \partial_{x} u_{0}\right\|_{L_{x y}^{2}}+\left\|\partial_{y}^{4} u_{0}\right\|_{L_{x y}^{2}}+\left\|\partial_{y}^{5} u_{0}\right\|_{L_{x y}^{2}}\right\} \\
\leq & C\left\|u_{0}\right\|_{Y} .
\end{aligned}
$$

Finally, Lemma 1 gives $\lambda_{3}\left(S(t) u_{0}\right) \leq C\left\|u_{0}\right\|_{Y}$.

Next, we bound $\Lambda_{T}(\mathbf{I I}(v))$. We start with $\lambda_{3}$, which contains three terms. For the first one, by Minkowski's inequality and Lemma 1, and then Holder's inequality,

$$
\begin{aligned}
& \left\|\partial_{x}^{-1} \partial_{y} \int_{0}^{t} S\left(t-t^{\prime}\right) \partial_{x} v \partial_{x}^{-1} \partial_{y} v d t^{\prime}\right\|_{L_{x}^{\infty} L_{y T}^{2}} \leq \int_{0}^{T}\left\|\partial_{x} v \partial_{x}^{-1} \partial_{y} v\right\|_{L_{x y}^{2}} \\
& \leq C T^{\frac{1}{2}}\left\|\partial_{x} v \partial_{x}^{-1} \partial_{y} v\right\|_{L_{x y T}^{2}} \leq C T^{\frac{1}{2}}\left\|\partial_{x} v\right\|_{L_{x}^{2} L_{y T}^{\infty}}\left\|\partial_{x}^{-1} \partial_{y} v\right\|_{L_{x}^{\infty} L_{y T}^{2}} \\
& \leq C T^{\frac{1}{2}} \lambda_{2}(v) \lambda_{3}(v) .
\end{aligned}
$$

For the second one, we proceed similarly. We will first bound the main terms, and then the errors using Lemma 4. We thus have

$$
\begin{aligned}
& \left\|\partial_{x}^{8} \partial_{x}^{-1} \partial_{y} \int_{0}^{t} S\left(t-t^{\prime}\right) \partial_{x} v \partial_{x}^{-1} \partial_{y} v d t^{\prime}\right\|_{L_{x}^{\infty} L_{y T}^{2}} \\
& \leq C \int_{0}^{T}\left\|\partial_{x} \partial_{x}^{8} v \partial_{x}^{-1} \partial_{y} v\right\|_{L_{x y}^{2}} d t+C \int_{0}^{T}\left\|\partial_{x} v \partial_{x}^{8} \partial_{x}^{-1} \partial_{y} v\right\|_{L_{x y}^{2}} d t+\text { errors } \\
& =\theta_{1}+\theta_{2}+\theta_{3} .
\end{aligned}
$$

To bound these terms, first observe

$$
\begin{aligned}
\left\|\partial_{x}^{-1} \partial_{y} v\right\|_{L_{x y}^{\infty}} & \leq C \sup _{t \in(0, T)}\left\{\left\|\partial_{x}^{-1} \partial_{y} v\right\|_{L_{x y}^{2}}+\left\|\partial_{y}^{2} \partial_{x}^{-1} \partial_{y} v\right\|_{L_{x y}^{2}}+\left\|\partial_{x}^{2} \partial_{x}^{-1} \partial_{y} v\right\|_{L_{x y}^{2}}\right\} \\
& \leq C \lambda_{1}(v) .
\end{aligned}
$$

Thus,

$$
\theta_{1} \leq C \lambda_{1}(v) \lambda_{4}(v) \int_{0}^{T} \frac{1}{t^{\frac{1}{4}}} d t \leq C T^{\frac{3}{4}} \lambda_{1}(v) \lambda_{4}(v)
$$

and

$$
\theta_{2} \leq C T^{\frac{1}{2}}\left\|\partial_{x} v \partial_{x}^{8} \partial_{x}^{-1} \partial_{y} v\right\|_{L_{x y T}^{2}} \leq C T^{\frac{1}{2}} \lambda_{2}(v) \lambda_{3}(v) .
$$

We now treat the error term $\theta_{3}$. It contains terms of the following form, for $m=$ $1, \ldots, 7$ :

$$
\int_{0}^{T}\left\|\left(\partial_{x} \partial_{x}^{m} v\right)\left(\partial_{x}^{8-m} \partial_{x}^{-1} \partial_{y} v\right)\right\|_{L_{x y}^{2}} d t
$$


We use Lemma 4, for $j \geq 3$ :

$$
\begin{aligned}
& \Delta_{j}\left(\left(\partial_{x}^{m} \partial_{x} v\right)\left(\partial_{x}^{8-m} \partial_{y} \partial_{x}^{-1} v\right)\right)=\Delta_{j}\left(\Delta_{0}\left(\partial_{x}^{m} \partial_{x} v\right) \Delta_{0}\left(\partial_{x}^{8-m} \partial_{x}^{-1} \partial_{y} v\right)\right) \\
& +\sum_{k \geq j-2} \Delta_{j}\left(\Delta_{k}\left(\partial_{x}^{m} \partial_{x} v\right) S_{k+1}\left(\partial_{x}^{8-m} \partial_{y} \partial_{x}^{-1} v\right)\right) \\
& +\sum_{k \geq j-2} \Delta_{j}\left(S_{k}\left(\partial_{x}^{m} \partial_{x} v\right) \Delta_{k}\left(\partial_{x}^{8-m} \partial_{x}^{-1} \partial_{y} v\right)\right) \\
& \equiv A_{j}+B_{j}+C_{j} .
\end{aligned}
$$

We now bound $B_{j}$. Note that

$$
\begin{aligned}
\mathcal{F} & \left(S_{k+1}\left(\partial_{x}^{8-m} \partial_{x}^{-1} \partial_{y} v\right)\right)=\varphi\left(\xi 2^{-(k+1)}\right) \mathcal{F}\left(\partial_{x}^{8-m} \partial_{x}^{-1} \partial_{y} v\right) \\
& =c \xi^{8-m}|\xi|^{-\frac{1}{4}} \varphi\left(\xi 2^{-(k+1)}\right) \mathcal{F}\left(D_{x}^{\frac{1}{4}} \partial_{x}^{-1} \partial_{y} v\right) \\
& =2^{k(8-m)} 2^{-\frac{k}{4}} \mathcal{F}\left(\tilde{S}_{k+1}\left(D_{x}^{\frac{1}{4}} \partial_{x}^{-1} \partial_{y} v\right)\right)
\end{aligned}
$$

for $\tilde{S}_{k+1}$, defined so that

$$
\mathcal{F}\left(\tilde{S}_{k+1} f\right)(\xi)=c 2^{-k(8-m)} \xi^{8-m} 2^{\frac{k}{4}}|\xi|^{-\frac{1}{4}} \varphi\left(\xi 2^{-(k+1)}\right)=\tilde{\varphi}\left(\xi 2^{-k}\right),
$$

where $\tilde{\varphi}(\mu)=c \mu^{8-m}|\mu|^{-\frac{1}{4}} \varphi\left(\frac{\mu}{2}\right)$. Thus

$$
\Delta_{k}\left(\partial_{x}^{m} \partial_{x} v\right) S_{k+1}\left(\partial_{x}^{8-m} \partial_{x}^{-1} \partial_{y} v\right)=2^{-\frac{k}{4}}\left(2^{k(8-m)} \Delta_{k}\left(\partial_{x} \partial_{x}^{m} v\right)\right) \tilde{S}_{k+1}\left(D_{x}^{\frac{1}{4}} \partial_{x}^{-1} \partial_{y} v\right) .
$$

Note that since $8-m \geq 1$ the following holds: $\hat{\tilde{\varphi}} \in L^{1}$.

Next, we have

$$
2^{k(8-m)} \Delta_{k}\left(\partial_{x}^{m} \partial_{x} v\right)=\tilde{\Delta}_{k}\left(\partial_{x}^{8} \partial_{x} v\right)
$$

where if $\widehat{\Delta_{k} f}(\xi)=\psi\left(2^{-k} \xi\right) \hat{f}(\xi)$, with $\psi$ supported in an annulus between $\frac{1}{4}$ and 4 , then

$$
\widehat{\widetilde{\Delta}_{k} f}(\xi)=\frac{2^{k(8-m)}}{\xi^{(k-m)}} \psi\left(2^{-k} \xi\right) \hat{f}(\xi)=\tilde{\psi}\left(2^{-k} \xi\right) \hat{f}(\xi),
$$

where $\tilde{\psi}$ has similar properties to $\psi$. Thus, we have

$$
\Delta_{k}\left(\partial_{x}^{m} \partial_{x} v\right) S_{k+1}\left(\partial_{x}^{8-m} \partial_{x}^{-1} \partial_{y} v\right)=2^{-\frac{k}{4}} \tilde{\Delta}_{k}\left(\partial_{x}^{8} \partial_{x} v\right) \tilde{S}_{k+1}\left(D_{x}^{\frac{1}{4}} \partial_{x}^{-1} \partial_{y} v\right),
$$

and we note that

$$
\begin{aligned}
\left\|D_{x}^{\frac{1}{4}} \partial_{x}^{-1} \partial_{y} v\right\|_{L_{x y T}^{\infty}} & \leq C \sup _{t \in(0, T)}\left\{\left\|\partial_{x}^{-1} \partial_{y} v\right\|_{L_{x y}^{2}}+\left\|\partial_{x}^{2} \partial_{x}^{-1} \partial_{y} v\right\|_{L_{x y}^{2}}+\left\|\partial_{y}^{2} \partial_{x}^{-1} \partial_{y} v\right\|_{L_{x y}^{2}}\right\} \\
& \leq C \lambda_{1}(v) .
\end{aligned}
$$

Then,

$$
\begin{aligned}
& \sum_{j \geq 3} \int_{0}^{T}\left\|B_{j}\right\|_{L_{x y}^{2}} d t \leq \sum_{j \geq 3} \sum_{k \geq j-2} 2^{-\frac{k}{4}} \int_{0}^{T}\left\|\tilde{\Delta}_{k}\left(\partial_{x}^{8} \partial_{x} v\right) \tilde{S}_{k+1}\left(D_{x}^{\frac{1}{4}} \partial_{x}^{-1} \partial_{y} v\right)\right\|_{L_{x y}^{2}} \\
& \leq \sum_{j \geq 3} \sum_{k \geq j-2} 2^{-\frac{k}{4}} \int_{0}^{T}\left\|\partial_{x}^{8} \partial_{x} v\right\|_{L_{x y}^{2}}\left\|D_{x}^{\frac{1}{4}} \partial_{x}^{-1} \partial_{y} v\right\|_{L_{x y}^{\infty}} \\
& \leq C \lambda_{1}(v) \lambda_{4}(v) \sum_{j \geq 3} \sum_{k \geq j-2} 2^{-\frac{k}{4}} \int_{0}^{T} \frac{d t}{t^{\frac{1}{4}}} \leq C T^{\frac{3}{4}} \lambda_{1}(v) \lambda_{4}(v) .
\end{aligned}
$$

We now turn to $C_{j}$. Here, we write

$$
S_{k}\left(\partial_{x}^{m} \partial_{x} v\right) \Delta_{k}\left(\partial_{x}^{8-m} \partial_{x}^{-1} \partial_{y} v\right)=2^{-k} \bar{S}_{k}\left(\partial_{x}^{2} v\right) \bar{\Delta}_{k}\left(\partial_{x}^{8} \partial_{x}^{-1} \partial_{y} v\right),
$$


for suitable $\bar{S}_{k}, \bar{\Delta}_{k}$, and where we have used $m \geq 1$. Then, we use

$$
\left\|\partial_{x}^{2} v\right\|_{L_{x}^{2} L_{y T}^{\infty}} \leq C\left\{\left\|\partial_{x} v\right\|_{L_{x}^{2} L_{y T}^{\infty}}+\left\|\partial_{x}^{3} v\right\|_{L_{x}^{2} L_{y T}^{\infty}}\right\} \leq C \lambda_{2}(v),
$$

to see that

$$
\begin{aligned}
& \sum_{j \geq 3} \int_{0}^{T}\left\|C_{j}\right\|_{L_{x y}^{2}} d t \leq C \sum_{j \geq 3} \sum_{k \geq j-2} 2^{-k} \int_{0}^{T}\left\|\bar{\Delta}_{k}\left(\partial_{x}^{8} \partial_{x}^{-1} \partial_{y} v\right) \bar{S}_{k}\left(\partial_{x}^{2} v\right)\right\|_{L_{x y}^{2}} d t \\
& \leq C \sum_{j \geq 3} \sum_{k \geq j-2} 2^{-k} T^{\frac{1}{2}}\left\|\bar{\Delta}_{k}\left(\partial_{x}^{8} \partial_{x}^{-1} \partial_{y} v\right) \bar{S}_{k}\left(\partial_{x}^{2} v\right)\right\|_{L_{x y T}^{2}} \\
& \leq C T^{\frac{1}{2}} \sum_{j \geq 3} \sum_{k \geq j-2} 2^{-k}\left\|\bar{\Delta}_{k}\left(\partial_{x}^{8} \partial_{x}^{-1} \partial_{y} v\right)\right\|_{L_{x}^{\infty} L_{y T}^{2}}\left\|\bar{S}_{k}\left(\partial_{x}^{2} v\right)\right\|_{L_{x}^{2} L_{y T}^{\infty}} \\
& \leq C T^{\frac{1}{2}} \sum_{j \geq 3} \sum_{k \geq j-2} 2^{-k}\left\|\partial_{x}^{8} \partial_{x}^{-1} \partial_{y} v\right\|_{L_{x}^{\infty} L_{y T}^{2}}\left\|\partial_{x}^{2} v\right\|_{L_{x}^{2} L_{y T}^{\infty}} \\
& \leq C T^{\frac{1}{2}} \lambda_{3}(v) \lambda_{2}(v) .
\end{aligned}
$$

Note also that for $j \geq 3$,

$$
A_{j}=\Delta_{j}\left(\Delta_{0}\left(\partial_{x}^{m+1} v\right) \Delta_{0}\left(\partial_{x}^{8-m} \partial_{x}^{-1} \partial_{y} v\right)\right)=0 .
$$

Using the proof of Lemma 4, for $j=0,1,2$, we still have

$$
\begin{aligned}
& \Delta_{j}\left(f_{1} f_{2}\right)=\Delta_{j}\left(\sum_{k=1}^{\infty} \Delta_{k}\left(f_{1}\right) S_{k+1}\left(f_{2}\right)\right) \\
& +\Delta_{j}\left(\sum_{k=1}^{\infty} S_{k}\left(f_{1}\right) \Delta_{k}\left(f_{2}\right)\right)+\Delta_{j}\left(\Delta_{0}\left(f_{1}\right) \Delta_{0}\left(f_{2}\right)\right),
\end{aligned}
$$

and the same arguments as above give the bounds

$$
\sum_{k=1}^{\infty} \int_{0}^{T}\left\|\Delta_{k}\left(\partial_{x}^{m} \partial_{x} v\right) S_{k+1}\left(\partial_{x}^{8-m} \partial_{x}^{-1} \partial_{y} v\right)\right\|_{L_{x y}^{2}} d t \leq C T^{\frac{3}{4}} \lambda_{1}(v) \lambda_{4}(v)
$$

and

$$
\sum_{k=1}^{\infty} \int_{0}^{T}\left\|S_{k}\left(\partial_{x}^{m} \partial_{x} v\right) \Delta_{k}\left(\partial_{x}^{8-m} \partial_{x}^{-1} \partial_{y} v\right)\right\|_{L_{x y}^{2}} d t \leq C T^{\frac{1}{2}} \lambda_{2}(v) \lambda_{3}(v) .
$$

Then, turning back to the error terms in $\theta_{3}$, we have

$$
\begin{aligned}
& \int_{0}^{T}\left\|\partial_{x}^{m} \partial_{x} v \partial_{x}^{8-m} \partial_{x}^{-1} \partial_{y} v\right\|_{L_{x y}^{2}} d t=\int_{0}^{T}\left\|\sum_{j \geq 0} \Delta_{j}\left(\left(\partial_{x}^{m} \partial_{x} v\right)\left(\partial_{x}^{8-m} \partial_{x}^{-1} \partial_{y} v\right)\right)\right\|_{L_{x y}^{2}} d t \\
& \leq \sum_{j=0}^{2} \int_{0}^{T}\left\|\Delta_{j}\left(\left(\partial_{x}^{m} \partial_{x} v\right)\left(\partial_{x}^{8-m} \partial_{x}^{-1} \partial_{y} v\right)\right)\right\|_{L_{x y}^{2}} d t \\
& \quad+\int_{0}^{T}\left\|\sum_{j \geq 3} \Delta_{j}\left(\left(\partial_{x}^{m} \partial_{x} v\right)\left(\partial_{x}^{8-m} \partial_{x}^{-1} \partial_{y} v\right)\right)\right\|_{L_{x y}^{2}} d t=F+G
\end{aligned}
$$


First,

$$
\begin{aligned}
F \leq & \sum_{j=0}^{2} \int_{0}^{T}\left\|\Delta_{j}\left(\Delta_{0}\left(\partial_{x}^{m} \partial_{x} v\right) \Delta_{0}\left(\partial_{x}^{8-m} \partial_{x}^{-1} \partial_{y} v\right)\right)\right\|_{L_{x y}^{2}} d t \\
& +\sum_{j=0}^{2} \int_{0}^{T}\left\|\Delta_{j}\left(\sum_{k=1}^{\infty} \Delta_{k}\left(\partial_{x}^{m} \partial_{x} v\right) S_{k+1}\left(\partial_{x}^{8-m} \partial_{x}^{-1} \partial_{y} v\right)\right)\right\|_{L_{x y}^{2}} d t \\
& +\sum_{j=0}^{2} \int_{0}^{T}\left\|\Delta_{j}\left(\sum_{k=1}^{\infty} S_{k}\left(\partial_{x}^{m} \partial_{x} v\right) \Delta_{k}\left(\partial_{x}^{8-m} \partial_{x}^{-1} \partial_{y} v\right)\right)\right\|_{L_{x y}^{2}} d t .
\end{aligned}
$$

Since $\left\|\Delta_{j}(f)\right\|_{L_{x y}^{2}} \leq\|f\|_{L_{x y}^{2}}$, the last two terms were controlled in a previous remark. For the first one, note that $\partial_{x}^{m} \Delta_{0}$ and $\partial_{x}^{8-m} \Delta_{0}$ are bounded operators (given by convolution with $L^{1}$ kernel). Thus, the first term is bounded by

$$
C T^{\frac{1}{2}}\left\|\partial_{x} v\right\|_{L_{x}^{2} L_{y T}^{\infty}}\left\|\partial_{x}^{-1} \partial_{y} v\right\|_{L_{x}^{\infty} L_{y T}^{2}} \leq C T^{\frac{1}{2}} \lambda_{2}(v) \lambda_{3}(v) .
$$

Finally, we turn to $G$, and we use our bounds on $B_{j}$ and $C_{j}$, and the observation that $\Delta_{j}\left(\Delta_{0}\left(f_{1}\right) \Delta_{0}\left(f_{2}\right)\right)=0$ for $j \geq 3$. This takes care of $\left\|\partial_{x}^{8} \partial_{x}^{-1} \partial_{y} v\right\|_{L_{x}^{\infty} L_{y T}^{2}}$.

In fact, $\left\|\partial_{y}^{8} \partial_{x}^{-1} \partial_{y} v\right\|_{L_{x}^{\infty} L_{y T}^{2}}$ is handled in a similar way, using Littlewood-Paley decomposition in the $y$ variable to handle the error terms. This then takes care of $\lambda_{3}$.

We next turn to $\lambda_{4}$. There are four terms. First, by using Lemma 3, we get

$t^{\frac{1}{4}}\left\|\partial_{x}^{8} \partial_{x} \int_{0}^{t} S\left(t-t^{\prime}\right) \partial_{x} v \partial_{x}^{-1} \partial_{y} v d t^{\prime}\right\|_{L_{x y}^{2}} \leq C t^{\frac{1}{4}} \int_{0}^{t} \frac{1}{\left|t-t^{\prime}\right|^{\frac{1}{4}}}\left\|\partial_{x}^{8}\left(\partial_{x} v \partial_{x}^{-1} \partial_{y} v\right)\right\|_{L_{x y}^{2}} d t^{\prime}$.

When using the Leibniz rule, we obtain two main terms:

$$
A+B=t^{\frac{1}{4}} \int_{0}^{t} \frac{1}{\left|t-t^{\prime}\right|^{\frac{1}{4}}}\left\|\partial_{x}^{9} v \partial_{x}^{-1} \partial_{y} v\right\|_{L_{x y}^{2}} d t^{\prime}+t^{\frac{1}{4}} \int_{0}^{t} \frac{1}{\left|t-t^{\prime}\right|^{\frac{1}{4}}}\left\|\partial_{x} v \partial_{x}^{8} \partial_{x}^{-1} \partial_{y} v\right\|_{L_{x y}^{2}} d t^{\prime}
$$

and error terms. We have

$$
\begin{aligned}
A & \leq t^{\frac{1}{4}} \int_{0}^{t} \frac{1}{\left|t-t^{\prime}\right|^{\frac{1}{4}}}\left\|\partial_{x}^{9} v\right\|_{L_{x y}^{2}}\left\|\partial_{x}^{-1} \partial_{y} v\right\|_{L_{x y}^{\infty}} d t^{\prime} \\
& \leq C t^{\frac{1}{4}}\left(\int_{0}^{t} \frac{1}{\left|t-t^{\prime}\right|^{\frac{1}{4}}\left|t^{\prime}\right|^{\frac{1}{4}}} d t^{\prime}\right) \lambda_{4}(v) \lambda_{1}(v) \\
& \leq C T^{\frac{3}{4}} \lambda_{4}(v) \lambda_{1}(v)
\end{aligned}
$$

and

$$
\begin{aligned}
B & \leq t^{\frac{1}{4}}\left(\int_{0}^{t} \frac{1}{\left|t-t^{\prime}\right|^{\frac{1}{2}}} d t^{\prime}\right)^{\frac{1}{2}}\left\|\partial_{x} v \partial_{x}^{8} \partial_{x}^{-1} \partial_{y} v\right\|_{L_{x y T}^{2}} \\
& \leq T^{\frac{3}{4}}\left\|\partial_{x} v\right\|_{L_{x}^{2} L_{y T}^{\infty}}^{\infty}\left\|\partial_{x}^{8} \partial_{x}^{-1} \partial_{y} v\right\|_{L_{x}^{\infty} L_{y T}^{2}} \leq T^{\frac{3}{4}} \lambda_{2}(v) \lambda_{3}(v)
\end{aligned}
$$


The error terms are handled by combining these estimates with the arguments we have used for the error terms in the case of $\lambda_{3}$. The treatment of

$$
\begin{gathered}
\partial_{x} \partial_{y}^{8} \int_{0}^{t} S\left(t-t^{\prime}\right) \partial_{x} v \partial_{x}^{-1} \partial_{y} v d t^{\prime}, \partial_{y} \partial_{x}^{8} \int_{0}^{t} S\left(t-t^{\prime}\right) \partial_{x} v \partial_{x}^{-1} \partial_{y} v d t^{\prime} \\
\partial_{y}^{9} \int_{0}^{t} S\left(t-t^{\prime}\right) \partial_{x} v \partial_{x}^{-1} \partial_{y} v d t^{\prime}
\end{gathered}
$$

is similar.

We next turn to $\lambda_{2}$. There are three terms. Using Lemma 2 and Minkowski's integral inequality, we first get

$$
\begin{aligned}
& \left\|\partial_{x} \int_{0}^{t} S\left(t-t^{\prime}\right) \partial_{x} v \partial_{x}^{-1} \partial_{y} v d t^{\prime}\right\|_{L_{x}^{2} L_{y T}^{\infty}} \leq C \int_{0}^{T}\left\|\partial_{x}\left(\partial_{x} v \partial_{x}^{-1} \partial_{y} v\right)\right\|_{L_{x y}^{2}} d t \\
& +C \int_{0}^{T}\left\|\partial_{x}^{6}\left(\partial_{x} v \partial_{x}^{-1} \partial_{y} v\right)\right\|_{L_{x y}^{2}} d t+C \int_{0}^{T}\left\|\partial_{x} \partial_{y}^{5}\left(\partial_{x} v \partial_{x}^{-1} \partial_{y} v\right)\right\|_{L_{x y}^{2}} d t \\
& +C \int_{0}^{T}\left\|\partial_{y}^{2}\left(\partial_{x} v \partial_{x}^{-1} \partial_{y} v\right)\right\|_{L_{x y}^{2}} d t+C \int_{0}^{T}\left\|\partial_{y}^{3}\left(\partial_{x} v \partial_{x}^{-1} \partial_{y} v\right)\right\|_{L_{x y}^{2}} d t .
\end{aligned}
$$

All these terms are controlled in the proof for $\lambda_{3}$.

Next, proceeding the same way we get

$$
\begin{aligned}
& \left\|\partial_{x}^{3} \int_{0}^{t} S\left(t-t^{\prime}\right) \partial_{x} v \partial_{x}^{-1} \partial_{y} v d t^{\prime}\right\|_{L_{x}^{2} L_{y T}^{\infty}} \leq C \int_{0}^{T}\left\|\partial_{x}^{3}\left(\partial_{x} v \partial_{x}^{-1} \partial_{y} v\right)\right\|_{L_{x y}^{2}} d t \\
& +C \int_{0}^{T}\left\|\partial_{x}^{8}\left(\partial_{x} v \partial_{x}^{-1} \partial_{y} v\right)\right\|_{L_{x y}^{2}} d t+C \int_{0}^{T}\left\|\partial_{x}^{3} \partial_{y}^{5}\left(\partial_{x} v \partial_{x}^{-1} \partial_{y} v\right)\right\|_{L_{x y}^{2}} d t \\
& +C \int_{0}^{T}\left\|\partial_{x}^{2} \partial_{y}^{2}\left(\partial_{x} v \partial_{x}^{-1} \partial_{y} v\right)\right\|_{L_{x y}^{2}} d t+C \int_{0}^{T}\left\|\partial_{x}^{2} \partial_{y}^{3}\left(\partial_{x} v \partial_{x}^{-1} \partial_{y} v\right)\right\|_{L_{x y}^{2}} d t
\end{aligned}
$$

terms which are again controlled in the proof for $\lambda_{3}$. The term

$$
\left\|\partial_{x} \partial_{y}^{2} \int_{0}^{t} S\left(t-t^{\prime}\right) \partial_{x} v \partial_{x}^{-1} \partial_{y} v d t^{\prime}\right\|_{L_{x}^{2} L_{y T}^{\infty}} \leq C \int_{0}^{T}\left\|\partial_{x}^{3}\left(\partial_{x} v \partial_{x}^{-1} \partial_{y} v\right)\right\|_{L_{x y}^{2}} d t
$$

is handled in the same way.

Finally, we turn to $\lambda_{1}$. There are six terms to estimate, and we start with the first three. Minkowski integral inequality gives for these three terms the following bound:

$$
\int_{0}^{T}\left\|\partial_{x} v \partial_{x}^{-1} \partial_{y} v\right\|_{L_{x y}^{2}}+\left\|\partial_{x}^{8}\left(\partial_{x} v \partial_{x}^{-1} \partial_{y} v\right)\right\|_{L_{x y}^{2}}+\left\|\partial_{y}^{8}\left(\partial_{x} v \partial_{x}^{-1} \partial_{y} v\right)\right\|_{L_{x y}^{2}} d t,
$$

and these terms are all controlled in the proof for $\lambda_{3}$. Now, by Lemma 1] (23),

$$
\begin{aligned}
& \left\|\partial_{x}^{-1} \partial_{y} \int_{0}^{t} S\left(t-t^{\prime}\right) \partial_{x} v \partial_{x}^{-1} \partial_{y} v d t^{\prime}\right\|_{L^{\infty}((0, T)) L_{x Y}^{2}} \leq C\left\|\partial_{x} v \partial_{x}^{-1} \partial_{y} v\right\|_{L_{x}^{1} L_{y T}^{2}} \\
& \leq C\left\|\partial_{x} v\right\|_{L_{x}^{2} L_{y T}^{\infty}}\left\|\partial_{x}^{-1} \partial_{y} v\right\|_{L_{x}^{2} L_{y T}^{2}} \leq C T^{\frac{1}{2}}\left\|\partial_{x} v\right\|_{L_{x}^{2} L_{y T}^{\infty}}\left\|\partial_{x}^{-1} \partial_{y} v\right\|_{L_{T}^{\infty} L_{x y}^{2}} \\
& \leq C T^{\frac{1}{2}} \lambda_{2}(v) \lambda_{1}(v) .
\end{aligned}
$$


Next, we have

$$
\begin{aligned}
& \left\|\partial_{x}^{2} \partial_{x}^{-1} \partial_{y} \int_{0}^{t} S\left(t-t^{\prime}\right) \partial_{x} v \partial_{x}^{-1} \partial_{y} v d t^{\prime}\right\|_{L^{\infty}((0, T)) L_{x y}^{2}} \leq C\left\|\partial_{x}^{2}\left(\partial_{x} v \partial_{x}^{-1} \partial_{y} v\right)\right\|_{L_{x}^{1} L_{y T}^{2}} \\
& \leq C\left\|\partial_{x}^{3} v \partial_{x}^{-1} \partial_{y} v\right\|_{L_{x}^{1} L_{y T}^{2}}+C\left\|\partial_{x}^{2} v \partial_{x} \partial_{x}^{-1} \partial_{y} v\right\|_{L_{x}^{1} L_{y T}^{2}}+C\left\|\partial_{x} v \partial_{x}^{2} \partial_{x}^{-1} \partial_{y} v\right\|_{L_{x}^{1} L_{y T}^{2}} .
\end{aligned}
$$

We have

$$
\begin{aligned}
& \left\|\partial_{x}^{3} v \partial_{x}^{-1} \partial_{y} v\right\|_{L_{x}^{1} L_{y T}^{2}} \leq\left\|\partial_{x}^{3} v\right\|_{L_{x}^{2} L_{y T}^{\infty}}\left\|\partial_{x}^{-1} \partial_{y} v\right\|_{L_{x}^{2} L_{y T}^{2}} \leq T^{\frac{1}{2}} \lambda_{2}(v) \lambda_{1}(v), \\
& \left\|\partial_{x} v \partial_{x}^{2} \partial_{x}^{-1} \partial_{y} v\right\|_{L_{x}^{1} L_{y T}^{2}} \leq\left\|\partial_{x} v\right\|_{L_{x}^{2} L_{y T}^{\infty}}\left\|\partial_{x}^{2} \partial_{x}^{-1} \partial_{y} v\right\|_{L_{x}^{2} L_{y T}^{2}} \leq T^{\frac{1}{2}} \lambda_{2}(v) \lambda_{1}(v), \\
& \left\|\partial_{x}^{2} v \partial_{x} \partial_{x}^{-1} \partial_{y} v\right\|_{L_{x}^{1} L_{y T}^{2}} \leq\left\|\partial_{x}^{2} v\right\|_{L_{x}^{2} L_{y T}^{\infty}}\left\|\partial_{x} \partial_{x}^{-1} \partial_{y} v\right\|_{L_{x}^{2} L_{y T}^{2}} \\
& \quad \leq\left\{\left\|\partial_{x} v\right\|_{L_{x}^{2} L_{y T}^{\infty}}+\left\|\partial_{x}^{3} v\right\|_{L_{x}^{2} L_{y T}^{\infty}}\right\}\left\{\left\|\partial_{x}^{-1} \partial_{y} v\right\|_{L_{x}^{2} L_{y T}^{2}}+\left\|\partial_{x}^{2} \partial_{x}^{-1} \partial_{y} v\right\|_{L_{x}^{2} L_{y T}^{2}}\right\} \\
& \leq T^{\frac{1}{2}} \lambda_{1}(v) \lambda_{2}(v) .
\end{aligned}
$$

The estimate for

$$
\left\|\partial_{y}^{2} \partial_{x}^{-1} \partial_{y} \int_{0}^{t} S\left(t-t^{\prime}\right) \partial_{x} v \partial_{x}^{-1} \partial_{y} v d t^{\prime}\right\|_{L^{\infty}((0, T)) L_{x y}^{2}}
$$

is analogous. (We need to use the inequality

$$
\left\|\partial_{y} f\right\|_{L_{x}^{2} L_{y T}^{\infty}} \leq C\left\{\|f\|_{L_{x}^{2} L_{y T}^{\infty}}+\left\|\partial_{y}^{2} f\right\|_{L_{x}^{2} L_{y T}^{\infty}}\right\}
$$

which is true.) This takes care of $\mathbf{I I}(v)$.

We now turn to $\Lambda_{T}(\mathbf{I}(v))$ which is easier. Recall that $\mathbf{I}(v)=\int_{0}^{t} S\left(t-t^{\prime}\right) \partial_{x}\left(v^{3}\right) d t^{\prime}$. Let us start with $\lambda_{1}$. First,

$$
\begin{aligned}
\left\|\partial_{x} \int_{0}^{t} S\left(t-t^{\prime}\right) v^{3} d t^{\prime}\right\|_{L_{x y}^{2}} & \leq \int_{0}^{t}\left\|v^{3}\right\|_{L_{x y}^{2}} \frac{d t^{\prime}}{\left|t-t^{\prime}\right|^{\frac{1}{4}}} \leq \int_{0}^{t}\|v\|_{L_{x y}^{\infty}}^{2}\|v\|_{L_{x y}^{2}} \frac{d t^{\prime}}{\left|t-t^{\prime}\right|^{\frac{1}{4}}} \\
& \leq C T^{\frac{3}{4}}\left(\lambda_{1}(v)\right)^{3} .
\end{aligned}
$$

Second,

$$
\left\|\partial_{x}^{8} \partial_{x} \int_{0}^{t} S\left(t-t^{\prime}\right) v^{3} d t^{\prime}\right\|_{L_{x y}^{2}} \leq \int_{0}^{t}\left\|\partial_{x}^{8}\left(v^{3}\right)\right\|_{L_{x y}^{2}} \frac{d t^{\prime}}{\left|t-t^{\prime}\right|^{\frac{1}{4}}}
$$

The main term is

$$
\int_{0}^{t}\left\|v^{2} \partial_{x}^{8} v\right\|_{L_{x y}^{2}} \frac{d t^{\prime}}{\left|t-t^{\prime}\right|^{\frac{1}{4}}} \leq T^{\frac{3}{4}}\left(\lambda_{1}(v)\right)^{3} .
$$

The intermediate terms can be handled using a variant of Lemma 4 for three functions. The term

$$
\left\|\partial_{y}^{8} \partial_{x} \int_{0}^{t} S\left(t-t^{\prime}\right) v^{3} d t^{\prime}\right\|_{L_{x y}^{2}}
$$

is handled similarly. Next, we turn to

$$
\left\|\partial_{x}^{-1} \partial_{y} \partial_{x} \int_{0}^{t} S\left(t-t^{\prime}\right) v^{3} d t^{\prime}\right\|_{L_{x y}^{2}}=\left\|\partial_{y} \int_{0}^{t} S\left(t-t^{\prime}\right) v^{3} d t^{\prime}\right\|_{L_{x y}^{2}} \leq \int_{0}^{t}\left\|v^{3}\right\|_{L_{x y}^{2}} \frac{d t^{\prime}}{\left|t-t^{\prime}\right|^{\frac{1}{2}}}
$$


which has already been handled. Next

$$
\begin{aligned}
& \left\|\partial_{x}^{2} \partial_{x}^{-1} \partial_{y} \partial_{x} \int_{0}^{t} S\left(t-t^{\prime}\right) v^{3} d t^{\prime}\right\|_{L_{x y}^{2}} \leq \int_{0}^{t}\left\|\partial_{x}^{2}\left(v^{3}\right)\right\|_{L_{x y}^{2}} \frac{d t^{\prime}}{\left|t-t^{\prime}\right|^{\frac{1}{4}}}, \\
& \left\|\partial_{y}^{2} \partial_{x}^{-1} \partial_{y} \partial_{x} \int_{0}^{t} S\left(t-t^{\prime}\right) v^{3} d t^{\prime}\right\|_{L_{x y}^{2}} \leq \int_{0}^{t}\left\|\partial_{y}^{2}\left(v^{3}\right)\right\|_{L_{x y}^{2}} \frac{d t^{\prime}}{\left|t-t^{\prime}\right|^{\frac{1}{4}}},
\end{aligned}
$$

which have already been handled.

We turn to $\lambda_{2}$. Here we again use Lemma 2 to get the result.

For $\lambda_{3}$, there are three terms. For the first term, we use Lemma 1 (22), and end up with

$$
\int_{0}^{T}\left\|\partial_{x}\left(v^{3}\right)\right\|_{L_{x y}^{2}} d t \leq \int_{0}^{T}\left\|\left(\partial_{x} v\right) v^{2}\right\|_{L_{x y}^{2}} d t \leq\|v\|_{L_{x y T}^{\infty}}^{2} T\|v\|_{L_{T}^{\infty} L_{x y}^{2}} \leq T\left(\lambda_{1}(v)\right)^{3} .
$$

For the second term, we again use Lemma 1 (22), and end up with

$$
\int_{0}^{T}\left\|\partial_{x}^{8} \partial_{x}\left(v^{3}\right)\right\|_{L_{x y}^{2}} d t
$$

The main term is controlled in the following way:

$$
\begin{aligned}
\int_{0}^{T}\left\|v^{2} \partial_{x}^{9} v\right\|_{L_{x y}^{2}} & \leq\|v\|_{L_{x y T}^{\infty}}^{2} \int_{0}^{T}\left\|\partial_{x}^{9} v\right\|_{L_{x y}^{2}} d t \leq\left(\lambda_{1}(v)\right)^{2}\left(\int_{0}^{t} \frac{d t}{t^{\frac{1}{4}}}\right) \lambda_{4}(v) \\
& \leq T^{\frac{3}{4}}\left(\lambda_{1}(v)\right)^{2} \lambda_{4}(v) .
\end{aligned}
$$

The next term is

$$
\int_{0}^{T}\left\|v \partial_{x} v \partial_{x}^{8} v\right\|_{L_{x y}^{2}} \leq\|v\|_{L_{x y T}^{\infty}}\left\|\partial_{x} v\right\|_{L_{x y T}^{\infty}} T\left\|\partial_{x}^{8} v\right\|_{L_{T}^{\infty} L_{x y}^{2}} \leq T\left(\lambda_{1}(v)\right)^{3} .
$$

To get the general term, we use Lemma 4. For the third term, we again use Lemma 1 (22) and end up with $\int_{0}^{T}\left\|\partial_{x}^{8} \partial_{x}\left(v^{3}\right)\right\|_{L_{x y}^{2}} d t$. This is handled similarly.

Finally, we turn to $\lambda_{4}$. There are four terms, the first one being

$$
t^{\frac{1}{4}}\left\|\partial_{x} \partial_{x}^{8} \int_{0}^{t} S\left(t-t^{\prime}\right) \partial_{x}\left(v^{3}\right) d t^{\prime}\right\|_{L_{x y}^{2}} \leq t^{\frac{1}{4}} \int_{0}^{t}\left\|\partial_{x}^{8} \partial_{x}\left(v^{3}\right)\right\|_{L_{x y}^{2}} \frac{d t^{\prime}}{\left|t-t^{\prime}\right|^{\frac{1}{4}}} .
$$

The main term is

$t^{\frac{1}{4}} \int_{0}^{t}\left\|v^{2} \partial_{x}^{9} v\right\|_{L_{x y}^{2}} \frac{d t^{\prime}}{\left|t-t^{\prime}\right|^{\frac{1}{4}}} \leq t^{\frac{1}{4}}\left(\int_{0}^{t} \frac{d t^{\prime}}{\left|t-t^{\prime}\right|^{\frac{1}{4}}\left|t^{\prime}\right|^{\frac{1}{4}}}\right)\|v\|_{L_{x y T}^{\infty}}^{2} \lambda_{4}(v) \leq T \lambda_{4}(v)\left(\lambda_{1}(v)\right)^{2}$.

The second term is

$t^{\frac{1}{4}} \int_{0}^{t}\left\|v^{2} \partial_{x} v \partial_{x}^{8} v\right\|_{L_{x y}^{2}} \frac{d t^{\prime}}{\left|t-t^{\prime}\right|^{\frac{1}{4}}} \leq T\|v\|_{L_{x y T}^{\infty}}\left\|\partial_{x} v\right\|_{L_{x y T}^{\infty}}\left\|\partial_{x}^{8} v\right\|_{L_{T}^{\infty} L_{x y}^{2}} \leq T\left(\lambda_{1}(v)\right)^{3}$.

The remaining terms are handled by a variant of Lemma 4. All other terms in $\lambda_{4}$ are similar.

Therefore, we have proved, for $0<T \leq 1$,

$$
\Lambda_{T}\left(\Phi_{u_{0}}(v)\right) \leq C\left\|u_{0}\right\|_{Y}+C T^{\frac{1}{2}}\left(\Lambda_{T}(v)\right)^{3}+C T^{\frac{1}{2}}\left(\Lambda_{T}(v)\right)^{2} .
$$

Moreover, similar arguments imply

$$
\Lambda_{T}\left(\Phi_{u_{0}}(v)-\Phi_{u_{0}}(\tilde{v})\right) \leq C T^{\frac{1}{2}} \Lambda_{T}(v-\tilde{v})\left(\left(\Lambda_{T}(v)\right)^{2}+\Lambda_{T}(v)+\left(\Lambda_{T}(\tilde{v})\right)^{2}+\Lambda_{T}(\tilde{v})\right) .
$$


Thus Claim 2 is proved for $\alpha=C\left\|u_{0}\right\|_{Y}+1$, and $T$ small enough depending only on $\left\|u_{0}\right\|_{Y}$. In particular, by a fixed point argument, we obtain a local existence result in $Y$, on a time interval $(0, T)$, where $T=T\left(\left\|u_{0}\right\|_{Y}\right)$.

We now prove a persistence property for smooth data.

For all $k, l \geq 2$, with $k \geq l+6$, we prove that if $u_{0} \in Y^{k, l}$, then the solution $u(t)$ constructed above belongs to $C\left([0, T], Y^{k, l}\right)$, for $T$ defined above. We argue as before; we define the following norms:

$$
\begin{aligned}
& \tilde{\lambda}_{1}(v)=\sup _{t \in[0, T]}\|v(t)\|_{Y^{k, l}}, \\
& \tilde{\lambda}_{2}(v)=\left\|\partial_{x} v\right\|_{L_{x}^{2} L_{y T}^{\infty}}+\left\|\partial_{x}^{l} \partial_{x} v\right\|_{L_{x}^{2} L_{y T}^{\infty}}+\left\|\partial_{y}^{l} \partial_{x} v\right\|_{L_{x}^{2} L_{y T}^{\infty},}, \\
& \tilde{\lambda}_{3}(v)=\left\|\partial_{x}^{-1} \partial_{y} v\right\|_{L_{x}^{\infty} L_{y T}^{2}}+\left\|\partial_{x}^{k} \partial_{x}^{-1} \partial_{y} v\right\|_{L_{x}^{\infty} L_{y T}^{2}}+\left\|\partial_{y}^{k} \partial_{x}^{-1} \partial_{y} v\right\|_{L_{x}^{\infty} L_{y T}^{2} T}, \\
& \tilde{\lambda}_{4}(v)=\sup _{t \in[0, T]} t^{\frac{1}{4}}\left\{\left\|\partial_{x} \partial_{x}^{k} v\right\|_{L_{x y}^{2}}+\left\|\partial_{x} \partial_{y}^{k} v\right\|_{L_{x y}^{2}}+\left\|\partial_{y} \partial_{x}^{k} v\right\|_{L_{x y}^{2}}+\left\|\partial_{x} \partial_{y}^{k} v\right\|_{L_{x y}^{2}}\right\}, \\
& \tilde{\Lambda}_{T}(v)=\max _{j=1, \ldots, 4} \tilde{\lambda}_{j}(v) .
\end{aligned}
$$

Using the same arguments, we prove, if $k \geq l+6$, that

$$
\tilde{\Lambda}_{T}\left(\Phi_{u_{0}}(u)\right) \leq C\left\|u_{0}\right\|_{Y^{k, l}}+C T^{\frac{1}{2}} \tilde{\Lambda}_{T}(u)\left(\Lambda_{T}^{2}(u)+\Lambda_{T}(u)\right),
$$

where $C$ may depend on $k, l$. The condition $k \geq l+6$ is needed to compensate the loss of derivative in Lemma 2. Therefore, there exists $T^{\prime}>0$ depending on $k, l$ such that

$$
\sup _{\left[0, T^{\prime}\right]}\|u(t)\|_{Y^{k, l}} \leq \tilde{\Lambda}_{T^{\prime}}(u) \leq 2 C\left\|u_{0}\right\|_{Y^{k, l}} .
$$

Iterating on the whole interval of existence $[0, T]$ of $u(t)$ in $Y$, we obtain the persistence property.

This completes the proof of Proposition 4

\section{LOCAL WELL-POSEDNESS OF THE MKP II EQUATION \\ VIA THE MiURA TRANSFORM}

We consider, for $\varepsilon \in(0,1)$, the following equation:

$$
\partial_{t} u+\varepsilon \partial_{x}^{4} u+\varepsilon^{5} \partial_{y}^{4} u+\partial_{x}^{3} u+3 \partial_{x}^{-1} \partial_{y}^{2} u-6 u^{2} \partial_{x} u+6 \partial_{x} u \partial_{x}^{-1} \partial_{y} u=0 .
$$

By Proposition 4 , we have the following result.

Corollary 5. Let $u_{0} \in Y^{\infty}$. There exists $T=T\left(\varepsilon,\left\|u_{0}\right\|_{Y}\right)>0$ and a unique solution $u$ of (29) satisfying

$$
u \in C\left([0, T], Y^{k, l}\right),
$$

for all $k, l \geq 0$, and (17) -196 .

Proof. Let $\varepsilon \in(0,1)$. Let $u_{0} \in Y^{\infty}$ and $\tilde{u}_{0}(x, y)=u_{0}\left(\varepsilon x, \varepsilon^{2} y\right)$. By Proposition 4, there exists a $\tilde{u} \in C\left([0, \tilde{T}], Y^{k, l}\right)$ solution of

$$
\partial_{t} \tilde{u}+\partial_{x}^{4} \tilde{u}+\partial_{y}^{4} \tilde{u}+\partial_{x}^{3} \tilde{u}+3 \partial_{x}^{-1} \partial_{y}^{2} \tilde{u}-6 \varepsilon^{2} \tilde{u}^{2} \partial_{x} \tilde{u}+6 \varepsilon^{2} \partial_{x} \tilde{u} \partial_{x}^{-1} \partial_{y} \tilde{u}=0
$$

on the time interval $\tilde{T}=\tilde{T}\left(\varepsilon,\left\|\tilde{u}_{0}\right\|_{Y}\right)$. Let

$$
u(t, x, y)=\tilde{u}\left(\varepsilon^{-3} t, \varepsilon^{-1} x, \varepsilon^{-2} y\right) .
$$

Then we check that $u$ is a solution of (29) with initial data $u_{0}$. This solution exists in $Y^{\infty}$ on the time interval $\left[0, T^{\prime}\right]$, where $T^{\prime}=\varepsilon^{3} \tilde{T}$. Since for fixed $\varepsilon>0$ we have 
$c_{1}\left\|u_{0}\right\|_{Y} \leq\left\|\tilde{u}_{0}\right\|_{Y} \leq c_{2}\left\|u_{0}\right\|_{Y}$, where $c_{1}, c_{2}>0$ depend on $\varepsilon$, the solution $u$ exists on a time interval $[0, T]$, where $T=T\left(\varepsilon,\left\|u_{0}\right\|_{Y}\right)>0$.

This section is organized as follows. In $\S 3.1$, we prove that the solution constructed in Corollary 5 can be extended to a time interval $[0, T]$, where $T=$ $T\left(\left\|u_{0}\right\|_{Z}\right)$ does not depend on $\varepsilon$, where $\left\|u_{0}\right\|_{Z}$ is a higher norm of $u_{0}$, and that this solution also satisfies uniform estimates on $[0, T]$. Next, in $\S 3.2$, we use these bounds to prove an existence result for the $\mathrm{mKP}$ equation by passing to the limit as $\varepsilon \rightarrow 0$.

3.1. Uniform time of existence by Miura transform and energy estimates. We consider the following functional space:

$$
Z=\left\{u \in H^{8}\left(\mathbf{R}^{2}\right): \partial_{x} u \in H^{8}\left(\mathbf{R}^{2}\right), \partial_{x}^{-1} \partial_{y} u \in H^{8}\left(\mathbf{R}^{2}\right)\right\} \subset Y,
$$

with the corresponding norm

$$
\|u\|_{Z}=\|u\|_{H^{8}}+\left\|\partial_{x} u\right\|_{H^{8}}+\left\|\partial_{x}^{-1} \partial_{y} u\right\|_{H^{8}}
$$

By energy estimates on the Miura transform of $u$, we claim the following lemma.

Lemma 5 (Energy estimate for the regularized mKP II equation). For any $u_{0} \in$ $Y^{\infty}$, there exist $T^{*}=T^{*}\left(\left\|u_{0}\right\|_{Z}\right)>0$ and $K=K\left(\left\|u_{0}\right\|_{Z}\right)>0$ such that if $u \in$ $C\left(\left[0, T^{\prime}\right], Y^{k, l}\right)$ for all $k, l \geq 0$, is a solution of the IVP (29) on $\left[0, T^{\prime}\right]$, where $0<T^{\prime} \leq T^{*}$, then for any $\varepsilon \in\left(0, \frac{1}{10}\right)$,

$$
\sup _{\left[0, T^{\prime}\right]}\|u(t)\|_{Z} \leq K
$$

Assuming this lemma, we have the following result.

Lemma 6 (Uniform time of existence). For any $u_{0} \in Y^{\infty}$, there exist $T^{*}=$ $T^{*}\left(\left\|u_{0}\right\|_{Z}\right)>0$ and $K=K\left(\left\|u_{0}\right\|_{Z}\right)>0$ such that, for any $\varepsilon \in\left(0, \frac{1}{10}\right)$ the IVP (29) has a unique solution $u_{\varepsilon} \in C\left(\left[0, T^{*}\right], Y^{k, l}\right)$, for all $k, l \geq 0$, satisfying

$$
\sup _{\left[0, T^{*}\right]}\left\|u_{\varepsilon}(t)\right\|_{Z} \leq K
$$

Proof. Let $\varepsilon \in\left(0, \frac{1}{10}\right)$. Let $u_{0} \in Y^{\infty}$. It follows from Corollary 5 that the IVP (29) has a unique solution $u_{\varepsilon}$ in $C\left(\left[0, T_{\varepsilon}\right], Y^{k, l}\right)$, for all $k, l \geq 0$, where $T_{\varepsilon}=T\left(\varepsilon,\left\|u_{0}\right\|_{Y}\right)$. If $T_{\varepsilon}<T^{*}$ ( $T^{*}$ being defined in Lemma 5 ), then Lemma 5 gives

$$
\sup _{\left[0, T_{\varepsilon}\right]}\left\|u_{\varepsilon}(t)\right\|_{Y} \leq \sup _{\left[0, T_{\varepsilon}\right]}\left\|u_{\varepsilon}(t)\right\|_{Z} \leq K\left(\left\|u_{0}\right\|_{Z}\right)
$$

Therefore, we can apply Corollary 5 as many times as necessary to extend the solution in $Y^{\infty}$ to the whole interval $\left[0, T^{*}\right]$. The bound (31) is then a consequence of (30).

We now prove Lemma 5 The reason why we use the space $Z$ here is that the space $Y$ is not adapted to the energy method through the Miura transform.

Proof of Lemma 5. The proof proceeds in three steps.

Step 1. Estimates through the Miura transform. First we recall some nonisotropic Sobolev inequalities that are proved in Besov et al. [1]. We give a short proof of these inequalities in the nonendpoint cases $(2<q<6)$ in Appendix C. 
Lemma 7 (Sobolev inequalities [1, Theorem 15.7). For any $q \in[2,6]$, there exists $K_{0}(q)>0$ such that for any $\varphi \in H^{2}\left(\mathbf{R}^{2}\right)$,

$$
\left\|\partial_{x} \varphi\right\|_{L^{q}}^{q} \leq K_{0}\left\|\partial_{x} \varphi\right\|_{L^{2}}^{\frac{6-q}{2}}\left\|\partial_{x}^{2} \varphi\right\|_{L^{2}}^{q-2}\left\|\partial_{y} \varphi\right\|_{L^{2}}^{\frac{q-2}{2}} .
$$

We also recall the following estimates (see Lemma 2.10 in [10] and [9], 5]).

Lemma 8. Let $s \geq 1$ and $1<p<\infty$. Then

$$
\left\|\left[J^{s}, f\right] g\right\|_{L^{p}} \leq C\left\{\|\nabla f\|_{L^{p_{1}}}\left\|J^{s-1} g\right\|_{L^{p_{2}}}+\left\|J^{s} f\right\|_{L^{p_{3}}}\|g\|_{L^{p_{4}}}\right\}
$$

and

$$
\left\|J^{s}(f g)\right\|_{L^{p}} \leq C\left\{\|f\|_{L^{p_{1}}}\left\|J^{s} g\right\|_{L^{p_{2}}}+\left\|J^{s} f\right\|_{L^{p_{3}}}\|g\|_{L^{p_{4}}}\right\},
$$

with $1<p_{2}, p_{3}<\infty$ such that $\frac{1}{p}=\frac{1}{p_{1}}+\frac{1}{p_{2}}=\frac{1}{p_{3}}+\frac{1}{p_{4}}$.

We claim the following result.

Lemma 9. Let $u \in Y^{\infty}$, and let $v \in H^{\infty}$ be such that

$$
v=\partial_{x} u+\partial_{x}^{-1} \partial_{y} u-u^{2} \text {. }
$$

Then,

$$
\left\|\partial_{x} u\right\|_{L^{2}} \leq\|v\|_{L^{2}}, \quad\left\|\partial_{x}^{-1} \partial_{y} u\right\|_{L^{2}} \leq C\left(\|u\|_{L^{2}}^{2}+\|v\|_{L^{2}}^{4}+1\right),
$$

and, for all integers $s \geq 0$, there exists $\alpha_{s} \geq 0$ and $C_{s} \geq 0$ such that

$$
\begin{aligned}
& \left\|\partial_{x}^{2} J^{s} u\right\|_{L^{2}}+\left\|\partial_{y} J^{s} u\right\|_{L^{2}} \leq 2\left\|\partial_{x} J^{s} v\right\|_{L^{2}}+C_{s}\left(1+\left\|J^{s} v\right\|_{L^{2}}+\|u\|_{L^{2}}\right)^{\alpha_{s}}, \\
& \left\|\partial_{x} J^{s} u\right\|_{L^{2}}+\left\|\partial_{x}^{-1} \partial_{y} J^{s} u\right\|_{L^{2}} \leq C_{s}\left(1+\left\|J^{s} v\right\|_{L^{2}}+\|u\|_{L^{2}}\right)^{\alpha_{s}} .
\end{aligned}
$$

Proof of Lemma 9. From (32), since $\int\left(\partial_{x} u\right) u^{2}=0$ and $\int\left(\partial_{x} u\right)\left(\partial_{x}^{-1} \partial_{y} u\right)=-\int u \partial_{y} u$ $=0$, we have

$$
\int v^{2}=\int\left(\partial_{x} u\right)^{2}+\int\left(\partial_{x}^{-1} \partial_{y} u-u^{2}\right)^{2},
$$

and so the first part of (33) holds. From (36), we also have

$$
\int\left(\partial_{x}^{-1} \partial_{y} u\right)^{2} \leq 2 \int\left\{\left(\partial_{x}^{-1} \partial_{y} u-u^{2}\right)^{2}+u^{4}\right\} \leq 2 \int v^{2}+2 \int u^{4} .
$$

Since, by Lemma 7

$$
\begin{aligned}
\int u^{4} & \leq C\left(\int\left(\partial_{x}^{-1} \partial_{y} u\right)^{2}\right)^{\frac{1}{2}}\left(\int u^{2}\right)^{\frac{1}{2}} \int\left(\partial_{x} u\right)^{2} \\
& \leq \frac{1}{4} \int\left(\partial_{x}^{-1} \partial_{y} u\right)^{2}+C\left(\int u^{2}\right)\left(\int\left(\partial_{x} u\right)^{2}\right)^{2} \\
& \leq \frac{1}{4} \int\left(\partial_{x}^{-1} \partial_{y} u\right)^{2}+C\left(\int u^{2}\right)\left(\int v^{2}\right)^{2},
\end{aligned}
$$

we obtain

$$
\int\left(\partial_{x}^{-1} \partial_{y} u\right)^{2} \leq 4 \int v^{2}+C\left(\int u^{2}\right)^{2}+C\left(\int v^{2}\right)^{4} .
$$

Thus (33) is proved. 
Proof of (34). Differentiating (32) with respect to $x$, we have $\partial_{x} v=\partial_{x}^{2} u+\partial_{y} u-$ $2 u \partial_{x} u$. By $\int\left(\partial_{x}^{2} u\right) \partial_{y} u=-\frac{1}{2} \int \partial_{y}\left(\partial_{x} u\right)^{2}=0$, we have, using Holder's inequality,

$$
\begin{aligned}
\int\left(\partial_{x}^{2} u\right)^{2}+\left(\partial_{y} u\right)^{2} & \leq 2 \int\left(\partial_{x} v\right)^{2}+8 \int\left(u \partial_{x} u\right)^{2} \\
& \leq 2 \int\left(\partial_{x} v\right)^{2}+C\left(\int u^{6}\right)^{\frac{1}{3}}\left(\int\left|\partial_{x} u\right|^{3}\right)^{\frac{2}{3}} .
\end{aligned}
$$

We recall the following two inequalities (the first one is contained in Lemma 7 , and the second is a standard Sobolev inequality in space dimension 2):

$$
\begin{aligned}
& \int\left|\partial_{x} u\right|^{3} \leq C\left(\int\left(\partial_{x} u\right)^{2}\right)^{\frac{3}{4}}\left(\int\left(\partial_{x}^{2} u\right)^{2}\right)^{\frac{1}{2}}\left(\int\left(\partial_{y} u\right)^{2}\right)^{\frac{1}{4}}, \\
& \int u^{6} \leq C\left(\int u^{2}\right)\left(\int\left(\partial_{x} u\right)^{2}\right)\left(\int\left(\partial_{y} u\right)^{2}\right) .
\end{aligned}
$$

Therefore

$$
\begin{aligned}
& \int\left(\left(\partial_{x}^{2} u\right)^{2}+\left(\partial_{y} u\right)^{2}\right) \\
& \leq 2 \int\left(\partial_{x} v\right)^{2}+C\left(\int u^{2}\right)^{\frac{1}{3}}\left(\int\left(\partial_{x} u\right)^{2}\right)^{\frac{5}{6}}\left(\int\left(\partial_{x}^{2} u\right)^{2}\right)^{\frac{1}{3}}\left(\int\left(\partial_{y} u\right)^{2}\right)^{\frac{1}{2}} \\
& \leq 2 \int\left(\partial_{x} v\right)^{2}+\frac{1}{2} \int\left(\partial_{y} u\right)^{2}+C\left(\int u^{2}\right)^{\frac{2}{3}}\left(\int\left(\partial_{x} u\right)^{2}\right)^{\frac{5}{3}}\left(\int\left(\partial_{x}^{2} u\right)^{2}\right)^{\frac{2}{3}} \\
& \leq 2 \int\left(\partial_{x} v\right)^{2}+\frac{1}{2} \int\left(\left(\partial_{x}^{2} u\right)^{2}+\left(\partial_{y} u\right)^{2}\right)+C\left(\int u^{2}\right)^{2}\left(\int\left(\partial_{x} u\right)^{2}\right)^{5} .
\end{aligned}
$$

Thus,

$$
\int\left(\left(\partial_{x}^{2} u\right)^{2}+\left(\partial_{y} u\right)^{2}\right) \leq 4 \int\left(\partial_{x} v\right)^{2}+C\left(\int v^{2}\right)^{10}+C\left(\int u^{2}\right)^{4} .
$$

This proves (34) for $s=0$. In particular, we have

$$
\begin{aligned}
\|u\|_{L^{\infty}}^{2}+\|J u\|_{L^{2}}^{2}+\left\|\partial_{x}^{2} u\right\|_{L^{2}}^{2} & \leq C \int\left(\left(\partial_{x}^{2} u\right)^{2}+\left(\partial_{y} u\right)^{2}+u^{2}\right) \\
& \leq C \int\left(\partial_{x} v\right)^{2}+C\left(\int v^{2}\right)^{10}+C\left(\int u^{2}\right)^{4}+C \int u^{2} .
\end{aligned}
$$

Proof of (34) for $s=1$. Arguing similarly, from $\partial_{x} J v=\partial_{x}^{2} J u+\partial_{y} J u-2 J\left(u \partial_{x} u\right)$ and the inequality

$$
\left\|J^{s}(f g)\right\|_{L^{2}} \leq C\left(\|f\|_{L^{\infty}}\left\|J^{s} g\right\|_{L^{2}}+\left\|J^{s} f\right\|_{L^{4}}\|g\|_{L^{4}}\right)
$$

(see Lemma 8), we have

$$
\begin{aligned}
\int\left(\left(\partial_{x}^{2} J u\right)^{2}+\left(\partial_{y} J u\right)^{2}\right) & \leq 2 \int\left(\partial_{x} J v\right)^{2}+8 \int\left(J\left(u \partial_{x} u\right)\right)^{2} \\
& \leq 2 \int\left(\partial_{x} J v\right)^{2}+C\|u\|_{L^{\infty}}^{2} \int\left(\partial_{x} J u\right)^{2}+C\left\|\partial_{x} u\right\|_{L^{4}}^{2}\|J u\|_{L^{4}}^{2} .
\end{aligned}
$$

On the one hand, since

$$
\int\left(\partial_{x} J u\right)^{2} \leq C\left(\int\left(\partial_{x}^{2} J u\right)^{2}\right)^{\frac{1}{2}}\left(\int(J u)^{2}\right)^{\frac{1}{2}},
$$


we have

$$
C\|u\|_{L^{\infty}}^{2} \int\left(\partial_{x} J u\right)^{2} \leq \frac{1}{8} \int\left(\partial_{x}^{2} J u\right)^{2}+C^{\prime}\|u\|_{L^{\infty}}^{4} \int(J u)^{2} .
$$

On the other hand, since

$$
\int w^{4} \leq C\left(\int\left(\partial_{x} w\right)^{2}\right)^{\frac{1}{2}}\left(\int\left(\partial_{y} w\right)^{2}\right)^{\frac{1}{2}} \int w^{2}
$$

we have

$$
\begin{aligned}
C\left\|\partial_{x} u\right\|_{L^{4}}^{2}\|J u\|_{L^{4}}^{2} & \leq C\left(\int\left(\partial_{x}^{2} u\right)^{2}\right)^{\frac{1}{4}}\left(\int\left(\partial_{y} J u\right)^{2}\right)^{\frac{1}{2}}\left(\int\left(\partial_{x} J u\right)^{2}\right)^{\frac{1}{4}} \int(J u)^{2} \\
\leq & C\left(\int\left(\partial_{x}^{2} u\right)^{2}\right)^{\frac{1}{4}}\left(\int\left(\partial_{y} J u\right)^{2}\right)^{\frac{1}{2}}\left(\int\left(\partial_{x}^{2} J u\right)^{2}\right)^{\frac{1}{8}}\left(\int(J u)^{2}\right)^{\frac{9}{8}} \\
& \leq \frac{1}{4} \int\left(\partial_{y} J u\right)^{2}+\frac{1}{8} \int\left(\partial_{x}^{2} J u\right)^{2}+C\left(\int\left(\partial_{x}^{2} u\right)^{2}\right)^{2}+C\left(\int(J u)^{2}\right)^{9} .
\end{aligned}
$$

Thus, from (39) and then (38), we obtain

$$
\begin{aligned}
& \int\left(\left(\partial_{x}^{2} J u\right)^{2}+\left(\partial_{y} J u\right)^{2}\right) \leq \\
& \leq 4 \int\left(\partial_{x} J v\right)^{2}+C\|u\|_{L^{\infty}}^{4} \int(J u)^{2}+C\left(\int\left(\partial_{x}^{2} u\right)^{2}\right)^{2}+C\left(\int(J u)^{2}\right)^{9} \\
& \leq 4 \int\left(\partial_{x} J v\right)^{2}+C\left(1+\int\left(\partial_{x} v\right)^{2}+\int v^{2}+\int u^{2}\right)^{\alpha},
\end{aligned}
$$

for some $\alpha>0$ ( $\alpha=90$ works). In particular,

$$
\begin{aligned}
& \left\|\partial_{x} u\right\|_{L^{\infty}}^{2}+\left\|\partial_{x}^{2} J u\right\|_{L^{2}}^{2}+\left\|J^{2} u\right\|_{L^{2}}^{2} \\
& \quad \leq C \int\left(\partial_{x} J v\right)^{2}+C\left(1+\int\left(\partial_{x} v\right)^{2}+\int v^{2}+\int u^{2}\right)^{\alpha} .
\end{aligned}
$$

Proof of (34) for $s \geq 2$. Let $s \geq 2$. From $\partial_{x} J^{s} v=\partial_{x}^{2} J^{s} u+\partial_{y} J^{s} u-2 J^{s}\left(u \partial_{x} u\right)$, using similar arguments, with

$$
\left\|J^{s}(f g)\right\|_{L^{2}} \leq C\left\{\|f\|_{L^{\infty}}\left\|J^{s} g\right\|_{L^{2}}+\left\|J^{s} f\right\|_{L^{2}}\|g\|_{L^{\infty}}\right\},
$$

we have

$$
\begin{aligned}
\int\left(\left(\partial_{x}^{2} J^{s} u\right)^{2}+\left(\partial_{y} J^{s} u\right)^{2}\right) \leq & 2 \int\left(\partial_{x} J^{s} v\right)^{2} \\
& +C\left\|\partial_{x} u\right\|_{L^{\infty}}^{2} \int\left(J^{s} u\right)^{2}+C\|u\|_{L^{\infty}}^{2} \int\left(\partial_{x} J^{s} u\right)^{2},
\end{aligned}
$$

and since

$$
C\|u\|_{L^{\infty}}^{2} \int\left(\partial_{x} J^{s} u\right)^{2} \leq \frac{1}{4} \int\left(\partial_{x}^{2} J^{s} u\right)^{2}+C\|u\|_{L^{\infty}}^{4} \int\left(J^{s} u\right)^{2},
$$

we obtain

$$
\int\left(\left(\partial_{x}^{2} J^{s} u\right)^{2}+\left(\partial_{y} J^{s} u\right)^{2}\right) \leq 4 \int\left(\partial_{x} J^{s} v\right)^{2}+C\left(\left\|\partial_{x} u\right\|_{L^{\infty}}^{2}+\|u\|_{L^{\infty}}^{4}\right) \int\left(J^{s} u\right)^{2} .
$$

The previous estimate, together with (38) and (40), allow us to prove (34) by induction on $s \geq 2$. 
Proof of (35). Let $s \geq 1$. From $J^{s} v=\partial_{x} J^{s} u+\partial_{x}^{-1} \partial_{y} J^{s} u-J^{s}\left(u^{2}\right)$, we have

$$
\int\left(\left(\partial_{x} J^{s} u\right)^{2}+\left(\partial_{x}^{-1} \partial_{y} J^{s} u\right)^{2}\right) \leq 2 \int\left(J^{s} v\right)^{2}+2 \int\left(J^{s}\left(u^{2}\right)\right)^{2} .
$$

Since $\left\|J^{s}\left(u^{2}\right)\right\|_{L^{2}} \leq C\left\|J^{s} u\right\|_{L^{2}}\|u\|_{L^{\infty}}$, we have

$$
\int\left(\left(\partial_{x} J^{s} u\right)^{2}+\left(\partial_{x}^{-1} \partial_{y} J^{s} u\right)^{2}\right) \leq 2 \int\left(J^{s} v\right)^{2}+C\left\|J^{s} u\right\|_{L^{2}}^{2}\|u\|_{L^{\infty}}^{2} .
$$

From (38) and (34), we obtain (35). This completes the proof of Lemma 9

Step 2. Energy method for the Miura transform of $u(t)$. We consider $u(t)$ defined on $\left[0, T^{\prime}\right]$ satisfying the assumptions of Lemma $[6$. First, we note directly on the equation of $u(t)$ that for $t \in\left[0, T^{\prime}\right]$,

$$
\int u^{2}(t) \leq \int u^{2}(0)
$$

Indeed, we have

$$
\frac{1}{2} \frac{d}{d t} \int u^{2}(t)=-\varepsilon \int\left(\left(\partial_{x}^{2} u(t)\right)^{2}+\varepsilon^{4}\left(\partial_{y}^{2} u(t)\right)^{2}\right) \leq 0 .
$$

The rest of the energy method cannot be performed directly on $u(t)$ because of the bilinear term in the equation of $u(t)$. Rather, we use the energy method on the Miura transform of $u(t)$.

Assume that $u(t)$ satisfies (29) and let

$$
v=\partial_{x} u+\partial_{x}^{-1} \partial_{y} u-u^{2} .
$$

Then $v(t)$ satisfies the following equation (see Appendix A for calculations):

$$
\begin{aligned}
& \partial_{x}\left(\partial_{t} v+\varepsilon \partial_{x}^{4} v+\varepsilon^{5} \partial_{y}^{4} v+\partial_{x}^{3} v+6 v \partial_{x} v\right)+3 \partial_{y}^{2} v \\
& \quad=-4 \partial_{x}\left[\varepsilon\left(\partial_{x}^{2}\left(\partial_{x} u\right)^{2}-\frac{1}{2}\left(\partial_{x}^{2} u\right)^{2}\right)+\varepsilon^{5}\left(\partial_{y}^{2}\left(\partial_{y} u\right)^{2}-\frac{1}{2}\left(\partial_{y}^{2} u\right)^{2}\right)\right] .
\end{aligned}
$$

The following lemma allows us to apply the energy method to $v(t)$.

Lemma 10. If $w \in C\left([0, T], H^{3}\right) \cap C^{1}\left([0, T], H^{-1}\right)$ solves $(a, b \geq 0)$

$$
\partial_{x}\left(\partial_{t} w+a \partial_{x}^{4} w+b \partial_{y}^{4} w+\partial_{x}^{3} w\right)+3 \partial_{y}^{2} w=\partial_{x} F,
$$

for some $F \in C\left([0, T], H^{2}\right)$, then $t \mapsto \int w^{2}(t)$ is a $C^{1}$ function of time and

$$
\frac{1}{2} \frac{d}{d t} \int w^{2}(t)=-a \int\left(\partial_{x}^{2} w(t)\right)^{2}-b \int\left(\partial_{y}^{2} w(t)\right)^{2}+\int F(t) w(t) .
$$

Proof of Lemma 10. First, we note that $w$ satisfies the Duhamel formula

$$
w(t)=\tilde{S}(t) w_{0}+\int_{0}^{t} \tilde{S}\left(t-t^{\prime}\right) F\left(t^{\prime}\right) d t^{\prime},
$$

where $\tilde{S}(t)$ is the solution operator for the linear part of equation (45):

$$
\tilde{S}(t) w_{0}=c \iint e^{i\left[x \xi+y \mu+t\left(\xi^{3}-3 \frac{\mu^{2}}{\xi}\right)\right]-a t \xi^{4}-b t \mu^{4}} \hat{w}_{0}(\xi, \mu) d \xi d \mu .
$$

Indeed, if $\tilde{w}(t)$ is the right-hand side of (47), then $\tilde{w}(t)$ satisfies (45), and if we set $z(t)=\partial_{x}(w(t)-\tilde{w}(t))$, then $z(t)$ satisfies $z(0)=0$ and

$$
\partial_{t} z+a \partial_{x}^{4} z+b \partial_{x}^{4} z+\partial_{x}^{3} z+3 \partial_{x}^{-1} \partial_{y}^{2} z=0 .
$$


Therefore, multiplying the equation by $z(t)$, we obtain $\frac{d}{d t} \int z^{2}(t) \leq 0$ and so $z \equiv 0$, so that $w \equiv \tilde{w}$.

Now, consider a smooth function $0 \leq \varphi_{n}(\xi) \leq 1$ which is 0 on $\left[-\frac{1}{n}, \frac{1}{n}\right]$ and 1 on $\mathbf{R} \backslash\left[-\frac{2}{n}, \frac{2}{n}\right]$. We define $w_{0 n}$ and $F_{n}$ such that $\hat{w}_{0 n}=\varphi_{n} \hat{w}(0)$ and $\hat{F}_{n}(t)=\varphi_{n} \hat{F}(t)$, and we consider $w_{n}(t)$ the solution of (45) corresponding to $w_{n}(0)=w_{0 n}$ and $F_{n}$ (such argument appears in Molinet 21]). The Duhamel formula implies that $w_{n}$ converges to $w$ as $n \rightarrow+\infty$ in $C\left([-T, T], H^{2}\right)$. Moreover, since $\partial_{x}^{-1} \partial_{y} w_{n}(t)$ is well defined, we have directly from the equation of $w_{n}(t)$

$$
\begin{aligned}
& \frac{1}{2}\left(\int w_{n}^{2}(t)-\int w_{n}^{2}(0)\right) \\
& \quad=\int_{0}^{t} \int\left\{-a\left(\partial_{x}^{2} w_{n}\left(t^{\prime}\right)\right)^{2}-b\left(\partial_{y}^{2} w_{n}\left(t^{\prime}\right)\right)^{2}+F_{n}\left(t^{\prime}\right) w_{n}\left(t^{\prime}\right)\right\} d t^{\prime} .
\end{aligned}
$$

Passing to the limit as $n \rightarrow+\infty$, we obtain the same formula for $w(t)$ which proves that $t \mapsto \int w^{2}(t)$ is a $C^{1}$ function and that (46) holds.

By the energy method applied to $v$, we have the following result.

Lemma 11. Under the assumptions of Lemma 5 , for all $\varepsilon \in\left(0, \frac{1}{10}\right)$ and for all $t \in\left[0, T^{\prime}\right]$

$$
\frac{d}{d t} \int\left(J^{8} v(t)\right)^{2} \leq C\left(\int\left(J^{8} v(t)\right)^{2}+\|u(t)\|_{L^{2}}^{2}+1\right)^{\gamma},
$$

for some constants $C, \gamma>0$ independent of $u_{0}$ and $\varepsilon$.

Proof. Let $s \geq 3$. We apply $J^{s}$ to equation (44), so that

$$
\begin{aligned}
\partial_{x} & \left(\partial_{t}\left(J^{s} v\right)+\varepsilon \partial_{x}^{4}\left(J^{s} v\right)+\varepsilon^{5} \partial_{y}^{4}\left(J^{s} v\right)+\partial_{x}^{3}\left(J^{s} v\right)\right. \\
& +6 v \partial_{x}\left(J^{s} v\right)+6\left[J^{s}, v\right] \partial_{x} v+3 \partial_{y}^{2}\left(J^{s} v\right) \\
= & -4 \partial_{x}\left[\varepsilon\left(\partial_{x}^{2} J^{s}\left(\partial_{x} u\right)^{2}-\frac{1}{2} J^{s}\left(\partial_{x}^{2} u\right)^{2}\right)+\varepsilon^{5}\left(\partial_{y}^{2} J^{s}\left(\partial_{y} u\right)^{2}-\frac{1}{2} J^{s}\left(\partial_{y}^{2} u\right)^{2}\right)\right]
\end{aligned}
$$

and we apply Lemma 10. We obtain after some integrations by parts

$$
\begin{aligned}
\frac{1}{2} \frac{d}{d t} \int\left(J^{s} v\right)^{2}= & -\varepsilon \int\left(\partial_{x}^{2} J^{s} v\right)^{2}-\varepsilon^{5} \int\left(\partial_{y}^{2} J^{s} v\right)^{2} \\
& +3 \int\left(\partial_{x} v\right)\left(J^{s} v\right)^{2}-6 \int\left(\left[J^{s}, v\right] \partial_{x} v\right) J^{s} v \\
& -4 \varepsilon \int J^{s}\left(\partial_{x} u\right)^{2}\left(\partial_{x}^{2} J^{s} v\right)+2 \varepsilon \int J^{s}\left(\partial_{x}^{2} u\right)^{2} J^{s} v \\
& -4 \varepsilon^{5} \int J^{s}\left(\partial_{y} u\right)^{2}\left(\partial_{y}^{2} J^{s} v\right)+2 \varepsilon^{5} \int J^{s}\left(\partial_{y}^{2} u\right)^{2} J^{s} v
\end{aligned}
$$

Control of (49). Since by Lemma 8 ,

$$
\left\|\left[J^{s}, f\right] g\right\|_{L^{2}} \leq C\left(\left(\left\|\partial_{x} f\right\|_{L^{\infty}}+\left\|\partial_{y} f\right\|_{L^{\infty}}\right)\left\|J^{s-1} g\right\|_{L^{2}}+\left\|J^{s} f\right\|_{L^{2}}\|g\|_{L^{\infty}}\right),
$$

we have

$$
\begin{aligned}
|[49)| & \leq C\left\|\partial_{x} v\right\|_{L^{\infty}}\left\|J^{s} v\right\|_{L^{2}}^{2}+C\left\|\left[J^{s}, v\right] \partial_{x} v\right\|_{L^{2}}\left\|J^{s} v\right\|_{L^{2}} \\
& \leq C\left(\left\|\partial_{x} v\right\|_{L^{\infty}}+\left\|\partial_{y} v\right\|_{L^{\infty}}\right) \int\left(J^{s} v\right)^{2} .
\end{aligned}
$$


Since $\left\|\partial_{x} v\right\|_{L^{\infty}}+\left\|\partial_{y} v\right\|_{L^{\infty}} \leq C\left\|J^{3} v\right\|_{L^{2}}$ and $s \geq 3$, we obtain

$$
|(49)| \leq C\left\|J^{s} v\right\|_{L^{2}}^{3} \text {. }
$$

Control of (50). First, we have

$$
\varepsilon\left|\int J^{s}\left(\partial_{x} u\right)^{2}\left(\partial_{x}^{2} J^{s} v\right)\right| \leq \int\left(J^{s}\left(\partial_{x} u\right)^{2}\right)^{2}+\varepsilon^{2} \int\left(\partial_{x}^{2} J^{s} v\right)^{2} .
$$

Since, by Lemma 8 ,

$$
\left\|J^{s}\left(f^{2}\right)\right\|_{L^{2}} \leq C\|f\|_{L^{\infty}}\left\|J^{s} f\right\|_{L^{2}},
$$

by (35) and $s \geq 3$, we obtain

$$
\begin{aligned}
\int\left(J^{s}\left(\partial_{x} u\right)^{2}\right)^{2} & \leq C\left\|\partial_{x} u\right\|_{L^{\infty}}^{2}\left\|\partial_{x} J^{s} u\right\|_{L^{2}}^{2} \leq C\left\|\partial_{x} J^{s} u\right\|_{L^{2}}^{4} \\
& \leq C\left(1+\left\|J^{s} v\right\|_{L^{2}}+\|u\|_{L^{2}}\right)^{4 \alpha_{s}}
\end{aligned}
$$

Second, by (53) and then (34),

$$
\begin{aligned}
\varepsilon\left|\int\left(J^{s}\left(\partial_{x}^{2} u\right)^{2}\right) J^{s} v\right| \leq & \varepsilon^{2}\left\|J^{s}\left(\partial_{x}^{2} u\right)^{2}\right\|_{L^{2}}^{2}+\left\|J^{s} v\right\|_{L^{2}}^{2} \\
\leq & \varepsilon^{2}\left\|\partial_{x}^{2} J^{s} u\right\|_{L^{2}}^{2}\left\|\partial_{x}^{2} u\right\|_{L^{\infty}}^{2}+\left\|J^{s} v\right\|_{L^{2}}^{2} \\
\leq & 4 \varepsilon^{2}\left\|\partial_{x} J^{s} v\right\|_{L^{2}}^{2}\left\|\partial_{x}^{2} u\right\|_{L^{\infty}}^{2}+C^{\prime}\left(\left\|J^{s} v\right\|_{L^{2}}+\|u\|_{L^{2}}+1\right)^{\alpha^{\prime}} \\
\leq & \varepsilon^{4}\left\|\partial_{x}^{2} J^{s} v\right\|_{L^{2}}^{2}+C\left\|J^{s} v\right\|_{L^{2}}^{2}\left\|\partial_{x}^{2} u\right\|_{L^{\infty}}^{4} \\
& +C^{\prime}\left(\left\|J^{s} v\right\|_{L^{2}}+\|u\|_{L^{2}}+1\right)^{\alpha^{\prime}} \\
\leq & \varepsilon^{4}\left\|\partial_{x}^{2} J^{s} v\right\|_{L^{2}}^{2}+C\left(\left\|J^{s} v\right\|_{L^{2}}+\|u\|_{L^{2}}+1\right)^{\alpha},
\end{aligned}
$$

for some $C, \alpha>0$.

Control of (51). First, by (53),

$$
\begin{aligned}
& \varepsilon^{5}\left|\int J^{s}\left(\partial_{y} u\right)^{2}\left(\partial_{y}^{2} J^{s} v\right)\right| \leq \varepsilon^{5}\left\|J^{s}\left(\partial_{y} u\right)^{2}\right\|_{L^{2}}\left\|\partial_{y}^{2} J^{s} v\right\|_{L^{2}} \\
& \quad \leq \varepsilon^{6}\left\|\partial_{y}^{2} J^{s} v\right\|_{L^{2}}^{2}+\varepsilon^{4}\left\|J^{s}\left(\partial_{y} u\right)^{2}\right\|_{L^{2}}^{2} \\
& \quad \leq \varepsilon^{6}\left\|\partial_{y}^{2} J^{s} v\right\|_{L^{2}}^{2}+C \varepsilon^{4}\left\|\partial_{y} u\right\|_{L^{\infty}}^{2}\left\|\partial_{y} J^{s} u\right\|_{L^{2}}^{2} \\
& \quad \leq \varepsilon^{6}\left\|\partial_{y}^{2} J^{s} v\right\|_{L^{2}}^{2}+C \varepsilon^{4}\left\|\partial_{y} u\right\|_{L^{\infty}}^{2}\left(\left\|\partial_{x} J^{s} v\right\|_{L^{2}}^{2}+\left(1+\left\|J^{s} v\right\|_{L^{2}}+\|u\|_{L^{2}}\right)^{\alpha_{s}}\right) \\
& \quad \leq \varepsilon^{6}\left\|\partial_{y}^{2} J^{s} v\right\|_{L^{2}}^{2}+C \varepsilon^{4}\left\|\partial_{y} u\right\|_{L^{\infty}}^{2}\left(\left\|\partial_{x}^{2} J^{s} v\right\|_{L^{2}}\left\|J^{s} v\right\|_{L^{2}}\right. \\
& \left.\quad+\left(1+\left\|J^{s} v\right\|_{L^{2}}+\|u\|_{L^{2}}\right)^{\alpha_{s}}\right) \\
& \quad \leq \varepsilon^{6}\left\|\partial_{y}^{2} J^{s} v\right\|_{L^{2}}^{2}+\varepsilon^{8}\left\|\partial_{x}^{2} J^{s} v\right\|_{L^{2}}^{2}+C\left(\left\|\partial_{y} u\right\|_{L^{\infty}}^{4}+1\right)\left(1+\left\|J^{s} v\right\|_{L^{2}}+\|u\|_{L^{2}}\right)^{\alpha^{\prime}} \\
& \leq \varepsilon^{6}\left\|\partial_{y}^{2} J^{s} v\right\|_{L^{2}}^{2}+\varepsilon^{8}\left\|\partial_{x}^{2} J^{s} v\right\|_{L^{2}}^{2}+C\left(1+\left\|J^{s} v\right\|_{L^{2}}+\|u\|_{L^{2}}\right)^{\alpha},
\end{aligned}
$$

for some $C, \alpha$. Second,

$$
\begin{aligned}
& \varepsilon^{5}\left|\int J^{s}\left(\partial_{y}^{2} u\right)^{2} J^{s} v\right|=\varepsilon^{5}\left|\int J^{s-2}\left(\partial_{y}^{2} u\right)^{2} J^{s+2} v\right| \\
& \quad \leq \varepsilon^{10}\left\|J^{s+2} v\right\|_{L^{2}}^{2}+\left\|J^{s-2}\left(\partial_{y}^{2} u\right)^{2}\right\|_{L^{2}}^{2} \\
& \quad \leq \varepsilon^{10}\left(2\left\|\partial_{x}^{2} J^{s} v\right\|_{L^{2}}^{2}+2\left\|\partial_{y}^{2} J^{s} v\right\|_{L^{2}}^{2}+C_{s}\|v\|_{L^{2}}^{2}\right)+C\left\|\partial_{y}^{2} u\right\|_{L^{\infty}}\left\|\partial_{y}^{2} J^{s-2} u\right\|_{L^{2}}^{2} . \\
& \quad \leq 2 \varepsilon^{10}\left(\left\|\partial_{x}^{2} J^{s} v\right\|_{L^{2}}^{2}+\left\|\partial_{y}^{2} J^{s} v\right\|_{L^{2}}^{2}\right)+C\left(1+\left\|J^{s} v\right\|_{L^{2}}+\|u\|_{L^{2}}\right)^{\alpha},
\end{aligned}
$$

for some $C, \alpha>0$. 
In conclusion, if $\varepsilon \in\left(0, \frac{1}{10}\right)$, for any $s \geq 3$, there exists $C_{s}>0$ and $\gamma_{s}>0$ such that

$$
\frac{d}{d t} \int\left(J^{s} v(t)\right)^{2} \leq C_{s}\left(\int\left(J^{s} v(t)\right)^{2}+\int u^{2}(t)+1\right)^{\gamma_{s}}
$$

Taking $s=8$ in the previous estimate, we have proved Lemma 11 .

Step 3. Conclusion of the energy method. From $v=\partial_{x} u+\partial_{x}^{-1} \partial_{y} u-u^{2}$, it is clear that

$$
\left\|J^{8} v(0)\right\|_{L^{2}} \leq C\left(\|u(0)\|_{Z}^{2}+1\right)
$$

From Lemma 11 and $\|u(t)\|_{L^{2}} \leq\|u(0)\|_{L^{2}}$, we have

$$
\frac{d}{d t} \int\left(J^{8} v(t)\right)^{2} \leq C\left(\int\left(J^{8} v(t)\right)^{2}+1\right)^{\gamma}
$$

for $C=C\left(\|u(0)\|_{L^{2}}\right)$. Thus, there exists $T^{*}=T^{*}\left(\|u(0)\|_{Z}\right)$ and $K_{1}=K_{1}\left(\|u(0)\|_{Z}\right)$ such that if the solution $u(t)$ is defined in $Z$ on $\left[0, T^{\prime}\right]$, with $0<T^{\prime}<T^{*}$, then

$$
\sup _{t \in\left[0, T^{\prime}\right]}\left\|J^{8} v(t)\right\|_{L^{2}}^{2} \leq K_{1} .
$$

Finally, by (34)-(35), we get

$$
\sup _{t \in\left[0, T^{\prime}\right]}\|u(t)\|_{Z}^{2} \leq K_{2}
$$

for some $K_{2}=K_{2}\left(K_{1}\right)=K_{2}\left(\|u(0)\|_{Z}\right)$. This concludes the proof of Lemma 5 .

3.2. Construction of local in time solution of the modified KP II equation. With the local existence result for the regularized mKP II equation and the uniform bound of Lemma 6. we are now ready to pass to the limit as $\varepsilon \rightarrow 0$ to build a strong solution of the mKP II equation.

Proposition 6. For any $u_{0} \in Y^{\infty}$, there exist $T^{*}=T^{*}\left(\left\|u_{0}\right\|_{Z}\right)$ such that the IVP (11) has a solution $u$ in the class $C\left(\left[0, T^{*}\right], H^{4}\left(\mathbf{R}^{2}\right)\right) \cap W^{1, \infty}\left(\left(0, T^{*}\right), H^{4}\left(\mathbf{R}^{2}\right)\right) \cap$ $L^{\infty}\left(\left(0, T^{*}\right), Z\right)$.

Proof. Let $u_{0} \in Y^{\infty}$. For a sequence $\varepsilon_{n} \rightarrow 0$, we consider the sequence of solutions $u_{n}$ of (29) in $C\left(\left[0, T^{*}\right], Y^{k, l}\right)$, for all $k, l \geq 0$, where $T^{*}=T^{*}\left(\left\|u_{0}\right\|_{Z}\right)$, given by Lemma 6. Note that by Lemma 6, we also have the uniform bound

$$
\sup _{\left[0, T^{*}\right]}\left\|u_{n}(t)\right\|_{Z} \leq K
$$

and by equation (29),

$$
\sup _{\left[0, T^{*}\right]}\left\|\partial_{t} u_{n}(t)\right\|_{H^{4}} \leq K .
$$

By classical arguments (see for example Lions [17, Chapter 1), there exists a subsequence of $u_{n}$, still denoted $u_{n}$, and a function $u \in L^{\infty}\left(\left(0, T^{*}\right), Z\right)$ with $\partial_{t} u \in L^{\infty}\left(\left(0, T^{*}\right), H^{4}(\mathbf{R})\right)$ and $w \in L^{\infty}\left(\left(0, T^{*}\right), H^{9}(\mathbf{R})\right)$ such that

$$
\begin{aligned}
& u_{n} \rightarrow u \quad \text { in } L^{\infty}\left(\left(0, T^{*}\right), H^{8}\left(\mathbf{R}^{2}\right)\right) \text { weak } *, \\
& \partial_{t} u_{n} \rightarrow \partial_{t} u \quad \text { in } L^{\infty}\left(\left(0, T^{*}\right), H^{4}\left(\mathbf{R}^{2}\right)\right) \text { weak } *, \\
& \partial_{x}^{-1} \partial_{y} u_{n} \rightarrow w \quad \text { in } L^{\infty}\left(\left(0, T^{*}\right), H^{9}\left(\mathbf{R}^{2}\right)\right) \text { weak } *,
\end{aligned}
$$


as $n \rightarrow+\infty$. It is clear that $w=\partial_{x}^{-1} \partial_{y} u$ since $\partial_{y} u_{n}$ converges to $\partial_{y} u$ and $\partial_{x}\left(\partial_{x}^{-1} \partial_{y} u_{n}\right)=\partial_{y} u_{n}$ converges to $\partial_{x} w$, so that $\partial_{x} w=\partial_{y} u$ and $w \in L^{2}(\mathbf{R})$ implies the result. Note also that by possibly taking a further subsequence,

$$
\begin{aligned}
& \partial_{x} u_{n} \rightarrow \partial_{x} u \quad \text { in } L_{l o c}^{2}\left(\left[0, T^{*}\right] \times \mathbf{R}^{2}\right) \text { strong, } \\
& \partial_{x}^{-1} \partial_{y} u_{n} \rightarrow \partial_{x}^{-1} \partial_{y} u \quad \text { in } L_{l o c}^{2}\left(\left[0, T^{*}\right] \times \mathbf{R}^{2}\right) \text { strong, }
\end{aligned}
$$

so that

$$
\partial_{x} u_{n} \partial_{x}^{-1} \partial_{y} u_{n} \rightarrow \partial_{x} u \partial_{x}^{-1} \partial_{y} u \quad \text { in } L_{l o c}^{1}\left(\left[0, T^{*}\right] \times \mathbf{R}^{2}\right) \text { strong, }
$$

as $n \rightarrow+\infty$. Moreover, $u \in C\left(\left[0, T^{*}\right], H^{4}\left(\mathbf{R}^{2}\right)\right)$, and $u_{n}(0) \rightarrow u(0)$ in $L^{2}\left(\mathbf{R}^{2}\right)$ weak as $n \rightarrow+\infty$, so that $u_{n}(0)=u_{0}$ makes sense (see Lemma 1.2 in [17], p. 7). It follows from what precedes that $u$ satisfies the IVP (11).

Remark 4. At this point we have not made much effort to obtain more information on the solution $u(t)$, since in the next section, using Bourgain's results for equation (9) and the Miura transform, we will very easily obtain all the information on $u(t)$. For example, regularity and uniqueness in a certain class will be straightforward.

4. Global Well-Posedness in the Energy space For the modified KP II EQUATION VIA THE MiURA TRANSFORM AND BOURGain's RESUlT

4.1. Bourgain's well-posedness result for KP II equation. Let $\langle a\rangle=$ $\left(1+a^{2}\right)^{\frac{1}{2}}$. For $b \in\left(\frac{1}{2}, 1\right), s \geq 0$, we define $X^{s, b}$ as the completion of the Schwartz space $\mathcal{S}\left(\mathbf{R}^{3}\right)$ with respect to the norm

$$
\|F\|_{X^{s, b}}=\left\|\left\langle\tau-\xi^{3}+3 \frac{\mu^{2}}{\xi}\right\rangle^{b}\left(1+|\xi|^{s}+|\mu|^{s}\right)\left(1+\frac{\left\langle\tau-\xi^{3}+3 \frac{\mu^{2}}{\xi}\right\rangle^{\frac{1}{4}}}{\langle\xi\rangle^{\frac{3}{4}}}\right) \hat{F}(\tau, \xi, \mu)\right\|_{L_{\tau \xi \mu}^{2}}
$$

(in the previous formula $\hat{F}$ denotes the Fourier transform in the three variables $(t, x, y))$. For $T>0$, we also define $X_{T}^{s, b}$ equipped with the norm

$$
\|F\|_{X_{T}^{s, b}}=\inf _{G \in X^{s, b}}\left\{\|G\|_{X^{s, b}}, G \equiv F \text { on }[0, T] \times \mathbf{R}^{2}\right\} .
$$

We recall the following result:

Theorem 7 (Bourgain [4). There exists $b \in\left(\frac{1}{2}, \frac{2}{3}\right)$ such that the following holds. Let $s \geq 0$. For any $v_{0} \in H^{s}\left(\mathbf{R}^{2}\right)$, there exists a function $v \in C\left(\mathbf{R}, H^{s}\left(\mathbf{R}^{2}\right)\right)$ which, for any $T>0$, is the unique solution of the IVP (9) on $[-T, T]$ satisfying

$$
v \in X_{T}^{s, b} \cap C\left([-T, T], H^{s}\left(\mathbf{R}^{2}\right)\right) .
$$

Moreover, if $v_{0}^{(1)}, v_{0}^{(2)} \in H^{s}\left(\mathbf{R}^{2}\right)$ and $v^{(1)}(t), v^{(2)}(t)$ are the corresponding global solutions of the IVP (2), then for all $0 \leq s^{\prime} \leq s$, there exists

$$
K_{s^{\prime}}=K_{s^{\prime}}\left(\left\|v_{0}^{(1)}\right\|_{H^{s^{\prime}}},\left\|v_{0}^{(2)}\right\|_{H^{s^{\prime}}}\right)
$$

such that

$$
\sup _{t \in[-1,1]}\left\|v^{(1)}(t)-v^{(2)}(t)\right\|_{H^{s^{\prime}}} \leq K_{s^{\prime}}\left\|v_{0}^{(1)}-v_{0}^{(2)}\right\|_{H^{s^{\prime}}} .
$$

Theorem 7 and the introduction of the $X^{s, b}$ spaces are due to Bourgain [4]. We also refer to [24] and [19] for related well-posedness results for $v_{0}$ in negative exponent Sobolev spaces and simpler proofs. 
Remark 5. Note that (56) does not imply uniqueness of the solution in the class $C\left(\mathbf{R}, H^{s}\left(\mathbf{R}^{2}\right)\right)$, since (56) holds only for the solutions constructed in the theorem. However, it is easy to see using classical arguments and Lemma 10 that uniqueness holds in the class $C\left([-T, T], H^{3}\right)$. Indeed, let $v^{(1)}, v^{(2)}$ be two solutions of (9) in $C\left([-T, T], H^{3}\right)$ such that $v^{(1)}(0)=v^{(2)}(0)=v_{0} \in H^{3}$. Then, if $w=v^{(1)}-v^{(2)}, w$ satisfies $w(0)=0$ and

$$
\partial_{x}\left(\partial_{t} w+\partial_{x}^{3} w+6 w \partial_{x} v^{(1)}+6 v^{(2)} \partial_{x} w\right)+3 \partial_{y}^{2} w=0 .
$$

By Lemma 10 with $a=b=0$, we have as usual

$$
\left|\frac{d}{d t} \int w^{2}(t)\right| \leq C\left(\left\|\partial_{x} v^{(1)}\right\|_{L^{\infty}}+\left\|\partial_{x} v^{(2)}\right\|_{L^{\infty}}\right) \int w^{2}(t),
$$

and since $\left\|\partial_{x} v\right\|_{L^{\infty}} \leq C\|v\|_{H^{3}}$, by Gronwall's lemma, we obtain $w \equiv 0$.

A first consequence of Theorem 7 on solutions of (1) is the following.

Proposition 8. For any $u_{0} \in Y^{\infty}$, there exists a unique solution $u \in C\left(\mathbf{R}, Y^{k, l}\right)$, for all $k, l>0$, of the IVP (11). Moreover, if we define $v$ by (3), then $v$ is the unique solution of the IVP (9). Finally, the following holds for all $t \in \mathbf{R}$ :

$$
\begin{aligned}
& \int u^{2}(t)=\int u_{0}^{2}, \\
& \int\left(\partial_{x} u(t)\right)^{2}+\int\left(\partial_{x}^{-1} \partial_{y} u-u^{2}\right)^{2}=E(u(t))=E\left(u_{0}\right) .
\end{aligned}
$$

Proof. Let $u_{0} \in Y^{\infty}$. We use Proposition [6] there exists $T^{*}=T^{*}\left(\left\|u_{0}\right\|_{Z}\right)$ and a local solution $u$ of the IVP (11) in the class $C\left(\left[0, T^{*}\right], H^{4}\right) \cap L^{\infty}\left(\left(0, T^{*}\right), Z\right)$. Let

$$
v=\partial_{x} u+\partial_{x}^{-1} \partial_{y} u-u^{2}
$$

Then $v \in L^{\infty}\left(\left(0, T^{*}\right), H^{8}\right)$, and by Appendix A, we check that $v$ is a solution of (91). Moreover, $\partial_{x} v=\partial_{x}^{2} u+\partial_{y} u-2 u \partial_{x} u \in C\left(\left[0, T^{*}\right], H^{2}\right)$, which gives a sense to $\partial_{x} v(0)$, and $\partial_{x} v$ satisfies the Duhamel formula

$$
\begin{aligned}
\partial_{x} v(t) & =W(t) \partial_{x} v(0)-6 \partial_{x} \int_{0}^{t} W\left(t-t^{\prime}\right)\left(v \partial_{x} v\right)\left(t^{\prime}\right) d t^{\prime} \\
& =\partial_{x}\left[W(t) v(0)-6 \int_{0}^{t} W\left(t-t^{\prime}\right)\left(v \partial_{x} v\right)\left(t^{\prime}\right) d t^{\prime}\right],
\end{aligned}
$$

where $W(t)$ is the group associated to the linear equation $\partial_{t} w+\partial_{x}^{3} w+3 \partial_{x}^{-1} \partial_{y}^{2} w=0$. Therefore $v(t)=W(t) v(0)-6 \int_{0}^{t} W\left(t-t^{\prime}\right)\left(v \partial_{x} v\right)\left(t^{\prime}\right) d t^{\prime}$, so $v \in C\left(\left[0, T^{*}\right], H^{7}\right)$.

By Remark 5, $v$ is the solution of the IVP (9) given by Bourgain's result. Therefore, it can be extended to a solution of (9) for all time, and since $v(0) \in H^{\infty}$, by Theorem 7 it satisfies $v \in C\left(\mathbf{R}, H^{s}\right)$, for all $s \geq 0$.

Let $\tilde{v}=-\partial_{x} u+\partial_{x}^{-1} \partial_{y} u-u^{2}$. It follows from Appendix A that $\tilde{v}$ is also a solution of (9), and similarly $\tilde{v}$ can be extended to a global solution $\tilde{v} \in C\left(\mathbf{R}, H^{s}\right)$, for all $s \geq 0$.

Thus $\partial_{x} u=\frac{1}{2}(v-\tilde{v}) \in C\left(\left[0, T^{*}\right], H^{\infty}\right)$. Moreover, since

$$
\partial_{y} u=\partial_{x}\left(u^{2}\right)+\frac{1}{2} \partial_{x}(v+\tilde{v})
$$

by induction, we obtain $u \in C\left(\left[0, T^{*}\right], H^{\infty}\right)$, and finally since $\partial_{x}^{-1} \partial_{y} u=u^{2}+$ $\frac{1}{2}(v+\tilde{v})$, we obtain $u \in C\left(\left[0, T^{*}\right], Y^{k, l}\right)$, for all $k, l \geq 0$. Now, we claim that $u$ 
can be extended to a global solution of the IVP (11) in $C\left(\mathbf{R}, Y^{k, l}\right)$, for all $k, l \geq 0$. Indeed, let $T>T^{*}$ arbitrary, and let $s \geq 10$. There exists $C=C(T, s)$ such that $\sup _{[0, T]}\|v(t)\|_{H^{s}} \leq C$. Thus, by Lemma 9, there exists $C^{\prime}=C^{\prime}(C)$ such that $\sup _{\left[0, T^{*}\right]}\|u(t)\|_{Z} \leq C$, and thus by Proposition $[$, $u$ can be extended to the interval $\left[0, T^{*}+\tau\right]$, where $\tau=\tau(C)>0$. Iterating this argument a finite number of times, $u(t)$ can be extended on $[0, T]$. Since $T$ was arbitrary, we have proved the claim.

Let us finish the proof of Proposition 8 by proving (57) and (58). First (57) follows easily from the equation of $u$ and Lemma 10 since

$$
\int u \partial_{x} u \partial_{x}^{-1} \partial_{y} u=-\frac{1}{2} \int u^{2} \partial_{y} u=0
$$

Second, (58) follows from the conservation law $\int v^{2}(t)=\int v^{2}(0)$ and the fact that

$$
\int\left(\partial_{x} u\right)\left(\partial_{x}^{-1} \partial_{y} u\right)=-\int u \partial_{y} u=0, \quad \int\left(\partial_{x} u\right) u^{2}=0 .
$$

Therefore Proposition 8 is proved.

4.2. Proof of Theorem $\mathbf{1}(\mathbf{i})-(\mathbf{v})$. Proposition 8 ensures the global well-posedness of the IVP for the mKP II equation for smooth data. Now, using the Miura transform and Theorem 7 , we prove uniform estimates in the energy space for these solutions. We approximate any data $u_{0} \in \mathcal{E}$ by a sequence $\left(u_{0 n}\right)$, where $u_{0 n} \in Y^{\infty}$, and then pass to the limit as $n \rightarrow+\infty$ to obtain a solution of (1) in the energy space $\mathcal{E}$.

Recall that we set

$\mathcal{E}=\left\{u \in L^{2}(\mathbf{R}),\|u\|_{\mathcal{E}}<\infty\right\}$, where $\|u\|_{\mathcal{E}}=\left\|\partial_{x} u\right\|_{L^{2}}+\|u\|_{L^{2}}+\left\|\partial_{x}^{-1} \partial_{y} u\right\|_{L^{2}}$.

The proof of Theorem 1 is based on the following lemma.

Lemma 12. There exists $\delta>0$ such that the following is true. Let $u_{0}^{(1)}, u_{0}^{(2)} \in Y^{\infty}$ and let $u^{(1)}, u^{(2)}$ be the unique solutions of the IVP (11) associated to $u_{0}^{(1)}$ and $u_{0}^{(2)}$, respectively. For all $A>0$, there exist $\theta=\theta(A)>0$ and $\tau=\tau(A)>0$ such that if, for $j \in\{1,2\}$,

$$
\left\|u^{(j)}\right\|_{L^{\infty}((0, \tau), \mathcal{E})} \leq A, \quad\left\|u^{(j)}\right\|_{L^{\infty}\left((0, \tau), L^{4}\right)} \leq \delta
$$

then

$$
\sup _{t \in[0, \tau]}\left\|u^{(1)}(t)-u^{(2)}(t)\right\|_{\mathcal{E}} \leq \theta\left\|u_{0}^{(1)}-u_{0}^{(2)}\right\|_{\mathcal{E}}
$$

Proof of Lemma 12. The proof of Lemma 12 is based on the Miura transform and (56) for $s^{\prime}=0$. Let $A>0$, and let $\tau \in(0,1)$ to be chosen later.

Step 1. Estimates of $\left\|v^{(1)}(t)-v^{(2)}(t)\right\|_{L^{2}}$. Let $u^{(1)}, u^{(2)}$ be the solutions of the IVP (11) associated to $u_{0}^{(1)}, u_{0}^{(2)} \in Y^{\infty}$, respectively. Assume that $u^{(1)}, u^{(2)}$ verify (59). For $j=1,2$, let

$$
v^{(j)}(t)=\partial_{x} u^{(j)}(t)+\partial_{x}^{-1} \partial_{y} u^{(j)}(t)-\left(u^{(j)}\right)^{2}(t),
$$

and in particular $v_{0}^{(j)}=v^{(j)}(0)$. Then, by Appendix A $v^{(j)}$ solves the KP II equation on $\mathbf{R}$ and by uniqueness, $v^{(j)}$ is the solution given by Theorem 7 . Note also that

$$
\left\|v_{0}^{(j)}\right\|_{L^{2}} \leq C(A) .
$$


In particular, by Theorem 7 , there exists a constant $\theta_{1}=\theta_{1}(A)>0$ such that

$$
\sup _{t \in[0,1]}\left\|v^{(1)}(t)-v^{(2)}(t)\right\|_{L^{2}} \leq \theta_{1}\left\|v_{0}^{(1)}-v_{0}^{(2)}\right\|_{L^{2}} .
$$

We have

(63) $v^{(1)}-v^{(2)}=\partial_{x}\left(u^{(1)}-u^{(2)}\right)+\partial_{x}^{-1} \partial_{y}\left(u^{(1)}-u^{(2)}\right)-\left(u^{(1)}-u^{(2)}\right)\left(u^{(1)}+u^{(2)}\right)$.

Thus, at $t=0$,

$$
\begin{aligned}
\left\|v_{0}^{(1)}-v_{0}^{(2)}\right\|_{L^{2}} \leq & \left\|\partial_{x}\left(u_{0}^{(1)}-u_{0}^{(2)}\right)\right\|_{L^{2}}+\left\|\partial_{x}^{-1} \partial_{y}\left(u_{0}^{(1)}-u_{0}^{(2)}\right)\right\|_{L^{2}} \\
& +\left\|\left(u_{0}^{(1)}-u_{0}^{(2)}\right)\left(u_{0}^{(1)}+u_{0}^{(2)}\right)\right\|_{L^{2}} \\
\leq & \left\|\partial_{x}\left(u_{0}^{(1)}-u_{0}^{(2)}\right)\right\|_{L^{2}}+\left\|\partial_{x}^{-1} \partial_{y}\left(u_{0}^{(1)}-u_{0}^{(2)}\right)\right\|_{L^{2}} \\
& +\left(\left\|u_{0}^{(1)}\right\|_{L^{4}}+\left\|u_{0}^{(2)}\right\|_{L^{4}}\right)\left\|u_{0}^{(1)}-u_{0}^{(2)}\right\|_{L^{4}} .
\end{aligned}
$$

By inequality (8), we have $\|w\|_{L^{4}} \leq c_{0}\|w\|_{\mathcal{E}}$, and so

$$
\left\|v_{0}^{(1)}-v_{0}^{(2)}\right\|_{L^{2}} \leq\left(1+c_{0}\left(\left\|u_{0}^{(1)}\right\|_{L^{4}}+\left\|u_{0}^{(2)}\right\|_{L^{4}}\right)\right)\left\|u_{0}^{(1)}-u_{0}^{(2)}\right\|_{\mathcal{E}}
$$

Thus by assumption (59),

$$
\left\|v_{0}^{(1)}-v_{0}^{(2)}\right\|_{L^{2}} \leq\left(1+2 c_{0} \delta\right)\left\|u_{0}^{(1)}-u_{0}^{(2)}\right\|_{\mathcal{E}},
$$

and for $\delta>0$ such that $c_{0} \delta<\frac{1}{2}$, we obtain

$$
\left\|v_{0}^{(1)}-v_{0}^{(2)}\right\|_{L^{2}} \leq 2\left\|u_{0}^{(1)}-u_{0}^{(2)}\right\|_{\mathcal{E}}
$$

Thus by (62), we obtain

$$
\sup _{t \in[0,1]}\left\|v^{(1)}(t)-v^{(2)}(t)\right\|_{L^{2}} \leq 2 \theta_{1}\left\|u_{0}^{(1)}-u_{0}^{(2)}\right\|_{\mathcal{E}} .
$$

Step 2. Control of $\partial_{x}\left(u^{(1)}-u^{(2)}\right)$ and $\partial_{x}^{-1} \partial_{y}\left(u^{(1)}-u^{(2)}\right)$ in $L^{2}$. By (63), since $\int \partial_{x} w \partial_{x}^{-1} \partial_{y} w=-\int w \partial_{y} w=0$, we have for all $t \in[0, \tau]$,

$$
\begin{aligned}
& \left\|\partial_{x}\left(u^{(1)}(t)-u^{(2)}(t)\right)\right\|_{L^{2}}+\left\|\partial_{x}^{-1} \partial_{y}\left(u^{(1)}(t)-u^{(2)}(t)\right)\right\|_{L^{2}} \\
& \quad \leq\left\|v^{(1)}(t)-v^{(2)}(t)\right\|_{L^{2}}+\left\|\left(u^{(1)}(t)-u^{(2)}(t)\right)\left(u^{(1)}(t)+u^{(2)}(t)\right)\right\|_{L^{2}} \\
& \quad \leq\left\|v^{(1)}(t)-v^{(2)}(t)\right\|_{L^{2}}+2 c_{0} \delta\left\|u^{(1)}(t)-u^{(2)}(t)\right\|_{\mathcal{E}} \\
& \quad \leq 2 \theta_{1}\left\|u_{0}^{(1)}-u_{0}^{(2)}\right\|_{\mathcal{E}}+2 c_{0} \delta\left\|u^{(1)}(t)-u^{(2)}(t)\right\|_{\mathcal{E}} .
\end{aligned}
$$

Therefore,

$$
\begin{aligned}
& \sup _{t \in[0, \tau]}\left\{\left\|\partial_{x}\left(u^{(1)}(t)-u^{(2)}(t)\right)\right\|_{L^{2}}+\left\|\partial_{x}^{-1} \partial_{y}\left(u^{(1)}(t)-u^{(2)}(t)\right)\right\|_{L^{2}}\right\} \\
& \quad \leq 2 \theta_{1}\left\|u_{0}^{(1)}-u_{0}^{(2)}\right\|_{\mathcal{E}}+2 c_{0} \delta \sup _{t \in[0, \tau]}\left\|u^{(1)}(t)-u^{(2)}(t)\right\|_{\mathcal{E}} .
\end{aligned}
$$

Step 3. Control of $u^{(1)}-u^{(2)}$ in $L^{2}$. Let $m \in C_{0}^{\infty}\left(\mathbf{R}^{2}\right)$ be such that

$$
0 \leq m \leq 1, \quad \operatorname{supp} m \subset\left\{|\xi|^{2}+|\mu|^{2} \leq 10\right\}, \quad m \equiv 1 \text { on }\{|\xi| \leq 1,|\mu| \leq 1\} .
$$

We define the operator $\mathbf{P}$ on $L^{2}\left(\mathbf{R}^{2}\right)$ by $\widehat{\mathbf{P f}}(\xi, \mu)=m(\xi, \mu) \hat{f}(\xi, \mu)$. We have $\|\mathbf{P} f\|_{L^{2}} \leq\|f\|_{L^{2}}$ and

$$
\|\mathbf{P} f\|_{L^{1}}+\left\|\partial_{x} \mathbf{P} f\right\|_{L^{1}}+\left\|\partial_{y} \mathbf{P} f\right\|_{L^{1}}+\left\|\partial_{x} \partial_{y} \mathbf{P} f\right\|_{L^{1}}+\left\|\partial_{x}^{2} \mathbf{P} f\right\|_{L^{1}} \leq C\|f\|_{L^{1}} .
$$


We set $w(t)=\mathbf{P} u^{(1)}(t)-\mathbf{P} u^{(2)}(t), w_{0}=\mathbf{P} u_{0}^{(1)}-\mathbf{P} u_{0}^{(2)}$. Then $w$ satisfies the following equation:

$$
\begin{aligned}
\partial_{t} w+\partial_{x}^{3} w+3 \partial_{x}^{-1} \partial_{y}^{2} w= & 2 \mathbf{P} \partial_{x}\left(\left(u^{(1)}\right)^{3}-\left(u^{(2)}\right)^{3}\right)-6 \mathbf{P}\left(\partial_{x}\left(u^{(1)}-u^{(2)}\right) \partial_{x}^{-1} \partial_{y} u^{(2)}\right) \\
& -6 \mathbf{P}\left(\partial_{x} u^{(1)} \partial_{x}^{-1} \partial_{y}\left(u^{(1)}-u^{(2)}\right)\right) \\
\equiv & h_{1}+h_{2}+h_{3} .
\end{aligned}
$$

Thus, $w(t)$ satisfies

$$
w(t)=W(t) w_{0}+\int_{0}^{t} W\left(t-t^{\prime}\right) \sum_{j=1}^{3} h_{j}\left(t^{\prime}\right) d t^{\prime},
$$

where $W(t)$ is the group associated to the linear equation $\partial_{t} w+\partial_{x}^{3} w+3 \partial_{y}^{2} \partial_{x}^{-1} w=0$. Recall that $\left\|W(t) w_{0}\right\|_{L^{2}}=\left\|w_{0}\right\|_{L^{2}}$. Thus,

$$
\sup _{t \in[0, \tau]}\|w(t)\|_{L^{2}} \leq\left\|u_{0}^{(1)}-u_{0}^{(2)}\right\|_{L^{2}}+\int_{0}^{\tau} \sum_{j=1}^{3}\left\|h_{j}\left(t^{\prime}\right)\right\|_{L^{2}} d t^{\prime} .
$$

From the previous estimate, we claim

$$
\sup _{t \in[0, \tau]}\|w(t)\|_{L^{2}} \leq\left\|u_{0}^{(1)}-u_{0}^{(2)}\right\|_{L^{2}}+C(A) \tau \sup _{t \in[0, \tau]}\left\|u^{(1)}(t)-u^{(2)}(t)\right\|_{\mathcal{E}} .
$$

Proof of (67). We need to control three terms. We recall the following GagliardoNirenberg inequality:

$$
\|f\|_{L^{2}} \leq \frac{1}{2}\left(\left\|\partial_{x} f\right\|_{L^{1}}+\left\|\partial_{y} f\right\|_{L^{1}}\right)
$$

First, by (68),

$$
\begin{aligned}
\left\|h_{1}\right\|_{L^{2}} & \leq C\left\|\partial_{x} h_{1}\right\|_{L^{1}}+C\left\|\partial_{y} h_{1}\right\|_{L^{1}} \\
& \leq C\left\|\partial_{x}^{2} \mathbf{P}\left(\left(u^{(1)}\right)^{3}-\left(u^{(2)}\right)^{3}\right)\right\|_{L^{1}}+C\left\|\partial_{y} \partial_{x} \mathbf{P}\left(\left(u^{(1)}\right)^{3}-\left(u^{(2)}\right)^{3}\right)\right\|_{L^{1}} \\
& \left.\leq C \|\left(u^{(1)}\right)^{3}-\left(u^{(2)}\right)^{3}\right)\left\|_{L^{1}} \leq C\right\| u^{(1)}-u^{(2)} \|_{L^{3}}\left(\left\|u^{(1)}\right\|_{L^{3}}^{2}+\left\|u^{(2)}\right\|_{L^{3}}^{2}\right) \\
& \leq C\left\|u^{(1)}-u^{(2)}\right\|_{\mathcal{E}}\left(\left\|u^{(1)}\right\|_{\mathcal{E}}^{2}+\left\|u^{(2)}\right\|_{\mathcal{E}}^{2}\right) .
\end{aligned}
$$

Thus,

Second,

$$
\left\|h_{1}(t)\right\|_{L^{2}} \leq C(A)\left\|u^{(1)}-u^{(2)}\right\|_{\mathcal{E}}
$$

$$
\begin{aligned}
\left\|h_{2}\right\|_{L^{2}} & =C\left\|\partial_{x} h_{2}\right\|_{L^{1}}+C\left\|\partial_{y} h_{2}\right\|_{L^{1}} \leq C\left\|\partial_{x}\left(u^{(1)}-u^{(2)}\right) \partial_{x}^{-1} \partial_{y} u^{(2)}\right\|_{L^{1}} \\
& \leq C\left\|\partial_{x}\left(u^{(1)}-u^{(2)}\right)\right\|_{L^{2}}\left\|\partial_{x}^{-1} \partial_{y} u^{(2)}\right\|_{L^{2}} \leq C(A)\left\|u^{(1)}-u^{(2)}\right\|_{\mathcal{E}} .
\end{aligned}
$$

We obtain a similar result for $\left\|h_{3}\right\|_{L^{2}}$. Thus (67) is proved.

Step 4. Conclusion of the proof of Lemma 12. Since, for any $f \in \mathcal{E}$,

$$
\|f\|_{L^{2}}^{2}=\int \hat{f}^{2} \leq \int(m \hat{f})^{2}+\int\left(\xi^{2}+\frac{\mu^{2}}{\xi^{2}}\right) \hat{f}^{2},
$$

we have

$$
\|f\|_{\mathcal{E}} \leq 2\left(\left\|\partial_{x} f\right\|_{L^{2}}+\left\|\partial_{x}^{-1} \partial_{y} f\right\|_{L^{2}}\right)+\|\mathbf{P} f\|_{L^{2}},
$$


and so gathering (66) and (67), we obtain

$$
\begin{aligned}
\sup _{t \in[0, \tau]}\left\|u^{(1)}(t)-u^{(2)}(t)\right\|_{\mathcal{E}} \leq & C\left\|u_{0}^{(1)}-u_{0}^{(2)}\right\|_{\mathcal{E}}+4 c_{0} \delta \sup _{t \in[0, \tau]}\left\|u^{(1)}(t)-u^{(2)}(t)\right\|_{\mathcal{E}} \\
& +C(A) \tau \sup _{t \in[0, \tau]}\left\|u^{(1)}(t)-u^{(2)}(t)\right\|_{\mathcal{E} .}
\end{aligned}
$$

Let $c_{0} \delta=\frac{1}{8}$; then

$\sup _{t \in[0, \tau]}\left\|u^{(1)}(t)-u^{(2)}(t)\right\|_{\mathcal{E}} \leq 2 C\left\|u_{0}^{(1)}-u_{0}^{(2)}\right\|_{\mathcal{E}}+2 C(A) \tau \sup _{t \in[0, \tau]}\left\|u^{(1)}(t)-u^{(2)}(t)\right\|_{\mathcal{E}}$

Choose $\tau=\tau(A)$ such that $2 C(A) \tau \leq \frac{1}{2}$. Then

$$
\sup _{t \in[0, \tau]}\left\|u^{(1)}(t)-u^{(2)}(t)\right\|_{\mathcal{E}} \leq 4 C\left\|u_{0}^{(1)}-u_{0}^{(2)}\right\|_{\mathcal{E}}
$$

which completes the proof of Lemma 12 ,

Now, we finish the proof of Theorem 1(i)-(iv). Let $u_{0} \in \mathcal{E}$, and let $\left(u_{0 n}\right)$ be a sequence of $Y^{\infty}$ such that $u_{0 n} \rightarrow u_{0}$ in $\mathcal{E}$ as $n \rightarrow+\infty$. Such a sequence is easily found by using the function $m$ defined in Step 3 of the proof of Lemma 12, Indeed, let $u_{0 n}$ be such that

$$
\hat{u}_{0 n}(\xi, \mu)=m\left(\frac{\xi}{n}, \frac{\mu}{n}\right) \hat{u}_{0}(\xi, \mu) .
$$

Then $u_{0 n} \in H^{s}$ for all $s \geq 0, u_{0 n} \rightarrow u_{0}$ in $L^{2}$ and $\partial_{x} u_{0 n} \rightarrow \partial_{x} u_{0}$ in $L^{2}$. Moreover, since $\frac{\mu}{\xi} \hat{u}_{0} \in L_{\xi \mu}^{2}, \partial_{x}^{-1} \partial_{y} u_{0 n}$ is well-defined in $H^{s}$ for all $s \geq 0$, by $\partial_{x}^{-1 \partial_{y} u_{0 n}}=$ $m\left(\frac{\xi}{n}, \frac{\mu}{n}\right) \frac{\mu}{\xi} \hat{u}_{0}$. Finally,

$$
\left\|\partial_{x}^{-1 \partial_{y} u_{0 n}}-\frac{\mu}{\xi} \hat{u}_{0}\right\|_{L^{2}} \rightarrow 0
$$

as $n \rightarrow+\infty$, and so $u_{0 n} \rightarrow u_{0}$ in $\mathcal{E}$ as $n \rightarrow+\infty$.

For all $n \geq 0$, we denote by $u_{n}$ the global smooth solution of (1) associated to $u_{0 n}$ given by Proposition 8 . Since $u_{0 n} \rightarrow u_{0}$ in $\mathcal{E}$, we may assume that for all $n \geq 0$, $\left\|u_{0 n}\right\|_{\mathcal{E}} \leq 2\left\|u_{0}\right\|_{\mathcal{E}} \equiv B$. By conservation of mass and energy (57)-(58), we claim that the following holds true:

$$
\forall n \geq 0, \quad \sup _{t \in \mathbf{R}}\left\|u_{n}(t)\right\|_{\mathcal{E}} \leq C(B) .
$$

Indeed, it is straightforward from (57)-(58) and Lemma 7 that

$$
\left\|u_{n}(t)\right\|_{L^{2}} \leq B, \quad\left\|\partial_{x} u_{n}(t)\right\|_{L^{2}} \leq C(B), \quad\left\|\partial_{x}^{-1} \partial_{y} u_{n}(t)-u_{n}^{2}\right\|_{L^{2}} \leq C(B) .
$$

From these estimates and Lemma 7 we have

$$
\begin{aligned}
\left\|\partial_{x}^{-1} \partial_{y} u_{n}(t)\right\|_{L^{2}} & \leq 2\left\|u_{n}(t)\right\|_{L^{4}}^{2}+C(B) \\
& \leq 2 C\left\|u_{n}(t)\right\|_{L^{2}}^{\frac{1}{2}}\left\|\partial_{x} u_{n}(t)\right\|_{L^{2}}\left\|\partial_{x}^{-1} \partial_{y} u_{n}(t)\right\|_{L^{2}}^{\frac{1}{2}}+C(B) \\
& \leq \frac{1}{2}\left\|\partial_{x}^{-1} \partial_{y} u_{n}(t)\right\|_{L^{2}}+C^{\prime}(B),
\end{aligned}
$$

and so $\left\|\partial_{x}^{-1} \partial_{y} u_{n}(t)\right\|_{L^{2}} \leq 2 C^{\prime}(B)$, so that (70) is proved.

Let $\lambda>0$ be chosen later. Let

$$
\tilde{u}_{n}(t, x, y)=\lambda u_{n}\left(\lambda^{3} t, \lambda x, \lambda^{2} y\right) .
$$


Then $\tilde{u}_{n}$ is still a solution of the IVP (11) corresponding to the initial data

$$
\tilde{u}_{0}(x, y)=\lambda u_{0 n}\left(\lambda x, \lambda^{2} y\right) .
$$

We have, for all $t \in \mathbf{R}$,

$$
\left\|\tilde{u}_{n}(t)\right\|_{L^{4}}=\lambda^{\frac{1}{4}}\left\|u_{n}(t)\right\|_{L^{4}} \leq \lambda^{\frac{1}{4}} C(B) .
$$

We choose $\lambda=\lambda(B)>0$ such that $\lambda^{\frac{1}{4}} C(B)<\delta$, where $\delta$ is defined in Lemma 12 , Such $\lambda$ being fixed, we have, for all $n \geq 0$, and for all $t \in \mathbf{R}$,

$$
\left\|\tilde{u}_{n}(t)\right\|_{\mathcal{E}}=\lambda^{-\frac{1}{2}}\left\|u_{n}(t)\right\|_{L^{2}}+\lambda^{\frac{1}{2}}\left\|\partial_{x} u_{n}(t)\right\|_{L^{2}}+\lambda^{\frac{1}{2}}\left\|\partial_{x}^{-1} \partial_{y} u_{n}(t)\right\|_{L^{2}} \leq C(B) .
$$

By Lemma 12, there exists $\tau=\tau(B)>0, \theta=\theta(B)>0$, such that for all $n, m \geq 0$, we have

$$
\sup _{t \in[0, \tau]}\left\|\tilde{u}_{n}(t)-\tilde{u}_{m}(t)\right\|_{\mathcal{E}} \leq \theta\left\|\tilde{u}_{0 n}(t)-\tilde{u}_{0 m}(t)\right\|_{\mathcal{E}} .
$$

Thus by uniform Cauchy convergence, there exists $\tilde{u} \in C([0, \tau], \mathcal{E})$ such that $\tilde{u}_{n} \rightarrow \tilde{u}$ in $L^{\infty}((0, \tau), \mathcal{E})$ as $n \rightarrow+\infty$. In particular, since $\tilde{u}_{n}(0)=\tilde{u}_{0 n} \rightarrow \tilde{u}_{0}$, we have $\tilde{u}(0)=\tilde{u}_{0}$.

Since $\partial_{x} \tilde{u}_{n} \rightarrow \partial_{x} \tilde{u}, \partial_{x}^{-1} \partial_{y} \tilde{u}_{n} \rightarrow \tilde{u}$ in $L^{2}$, and $\tilde{u}_{n} \rightarrow \tilde{u}$ in $L^{4}$, we have $\tilde{u}_{n}^{2} \partial_{x} \tilde{u}_{n} \rightarrow$ $\tilde{u}^{2} \partial_{x} \tilde{u}$ and $\partial_{x} \tilde{u}_{n} \partial_{x}^{-1} \partial_{y} \tilde{u}_{n} \rightarrow \partial_{x} \tilde{u} \partial_{x}^{-1} \partial_{y} \tilde{u}$ in $L^{1}$ as $n \rightarrow+\infty$. Therefore, $\tilde{u}$ satisfies equation (11) on $[0, \tau]$. Since $\tau$ depends only on $B$, and the approximate solutions are global, we can extend $\tilde{u}(t)$ to $\mathbf{R}$, to obtain a global solution of (11) in $\mathcal{E}$. We define $u(t, x, y)=\lambda^{-1} \tilde{u}\left(\lambda^{-3} t, \lambda^{-1} x, \lambda^{-2} y\right)$. Then, $u \in C(\mathbf{R}, \mathcal{E}) \cap C^{1}\left(\mathbf{R}, H^{-2}\right)$ solves the IVP (11), and is the limit of the sequence $\left(u_{n}\right)$. This proves the existence result. Theorem 1(v) follows from Proposition 8.

Proof of (i). We know that if $v_{n}$ is associated to $u_{n}$ by the Miura transform (3), then $v_{n}$ is the unique smooth solution of (9) with initial data $v_{n}(0)$, given by Theorem 7. Let $v_{0}=\partial_{x} u_{0}+\partial_{x}^{-1} \partial_{y} u_{0}-u_{0}^{2}$. By Theorem 7 we known that $v_{n} \rightarrow v$ in $L_{l o c}^{\infty}\left(\mathbf{R}, L^{2}\left(\mathbf{R}^{2}\right)\right)$, where $v(t)$ is the unique solution of (9) given by Theorem 7 with initial data $v_{0}$. Passing to the limit in the Miura transform relating $u_{n}$ and $v_{n}$, we obtain

$$
v(t)=\partial_{x} u(t)+\partial_{x}^{-1} \partial_{y} u(t)-u^{2}(t)
$$

This justifies (i) in the statement of Theorem 1 A similar statement holds for $\tilde{v}(t)=-\partial_{x} u(t)+\partial_{x}^{-1} \partial_{y} u(t)-u^{2}(t)$; see Appendix A.

Proof of (ii). If $\partial_{x} u_{0} \in H^{s}$ and $\partial_{x}^{-1} \partial_{y} u_{0} \in H^{s}$, for $s \geq 1$, then in particular $\partial_{y} u_{0} \in$ $H^{s-1}$ and so $u_{0} \in H^{s}$, and by $\partial_{x} u_{0} \in H^{s}$ again, we have $u_{0} \in L^{\infty}$, so that $u_{0}^{2} \in H^{s}$. Therefore $v_{0}=\partial_{x} u_{0}+\partial_{x}^{-1} \partial_{y} u_{0}-u_{0}^{2} \in H^{s}, \tilde{v}_{0}=-\partial_{x} u_{0}+\partial_{x}^{-1} \partial_{y} u_{0}-u_{0}^{2} \in H^{s}$ and by Theorem [7, we have $v, \tilde{v} \in C\left(\mathbf{R}, H^{s}\right)$.

A first consequence is $\partial_{x} u=\frac{1}{2}(v-\tilde{v})$ belongs to $C\left(\mathbf{R}, H^{s}\right)$. Next, we have for all time $\frac{1}{2} \partial_{x}(v+\tilde{v})=\partial_{y} u-2 u \partial_{x} u \in H^{s-1}$. Since $s \geq 1$, we have that $\partial_{x} u \in H^{s}$ implies $\partial_{x} u \in L^{4}$, and by Lemma 7, $u \in \mathcal{E}$ implies $u \in L^{4}$, and so $u \partial_{x} u \in L^{2}$. Thus $\partial_{y} u \in L^{2}$. Since $\partial_{x}^{2} u \in L^{2}$ and $\partial_{y} u \in L^{2}$, we have $u \in L^{\infty}$, and so $u \partial_{x} u \in H^{s}$, so that $\partial_{y} u \in H^{s-1}$. Thus $u \in H^{s}$ and $u^{2} \in H^{s}$ as well. Therefore, by $\frac{1}{2}(v+\tilde{v})=\partial_{x}^{-1} \partial_{y} u-u^{2} \in H^{s}$, we have $\partial_{x}^{-1} \partial_{y} u \in H^{s}$. Continuity in time is easily obtained by similar arguments.

Proof of (iii). It is a consequence of Lemma 12 and the scaling argument used for the proof of existence above. 
Proof of (iv). Let $u_{1}, u_{2}$ be two solutions of (11) in $C\left(\mathbf{R}, Y^{3,3}\right)$ associated to the same initial data $u_{0} \in Y^{3,3}$. Then, $v_{1}, v_{2} \in C\left(\mathbf{R}, H^{3}\right)$ are two solutions of (9) with same initial data. By Remark 5 concerning uniqueness for the KP II equation, we have $v_{1}=v_{2}$. We also have $\tilde{v}_{1}=\tilde{v}_{2}$. Thus $\partial_{x} u_{1}=\partial_{x} u_{2}=\frac{1}{2}\left(v_{1}-\tilde{v}_{1}\right)$, and so $u_{1} \equiv u_{2}$.

\section{Remark on the QUALitative Behavior of the SOlutions}

The objective of this section is to prove the last statement of Theorem 1 concerning the behavior of $t \rightarrow+\infty$ of the solution $u(t)$ of (11) constructed in the previous section. We state the result in the following proposition.

Proposition 9. Let $u_{0} \in \mathcal{E}$ and let $u \in C(\mathbf{R}, \mathcal{E})$ be the solution constructed in Theorem 11. Then, for any $\beta>0$, the following holds:

$$
\lim _{t \rightarrow+\infty} \int_{x>\beta t}\left\{\left(\partial_{x} u(t, x, y)\right)^{2}+\left(\partial_{x}^{-1} \partial_{y} u(t, x, y)\right)^{2}+u^{2}(t, x, y)\right\} d x d y=0 .
$$

Remark 6. By similar arguments, the same property is true for $H^{1}$ solutions of the modified KdV equation (4).

The proof is based on variants of the so-called Kato identity first written by Kato 8 for the generalized KdV equations. We need such identities for both the KP II and the mKP II equations. Originally, the Kato identity was introduced to prove a local smoothing effect of the KdV equation. In Martel and Merle [18, it is used to study qualitative properties of solutions, such as asymptotic properties in large time. The Kato identity for KP II was already used in this context in 2] (see also Saut 23]).

We claim the following lemma.

Lemma 13 (Kato identity for mKP II and KP II equations). Let $f=f(x) \in$ $C^{3}(\mathbf{R}, \mathbf{R})$. Let $u(t)$ be a solution of the $m K P$ II equation in $\mathcal{E}$ as given in Theorem 1. Then

$$
\frac{d}{d t} \int u^{2} f=-3 \int\left\{\left(\partial_{x} u\right)^{2}+\left(\partial_{x}^{-1} \partial_{y} u-u^{2}\right)^{2}\right\} f^{\prime}+\int u^{2} f^{\prime \prime \prime}
$$

Let $v(t)$ be a solution of the KP II equation in $\mathcal{E}$ as given in Theorem 7 , Then

$$
\frac{d}{d t} \int v^{2} f=-3 \int\left\{\left(\partial_{x} v\right)^{2}+\left(\partial_{x}^{-1} \partial_{y} v\right)^{2}-4 v^{3}\right\} f^{\prime}+\int v^{2} f^{\prime \prime \prime} .
$$

Note that for $f(x)=x$, if the quantities involved make sense, we have the following remarkable Virial type relation:

$$
\frac{d}{d t} \int x u^{2}=-3 E(u(0)),
$$

where the energy $E(u(0))$ is defined in the Introduction. 
Proof of Lemma 13. Proof of (73). We have by direct calculations for sufficiently regular solutions:

$$
\begin{aligned}
\frac{1}{2} & \frac{d}{d t} \int u^{2} f=\int u \partial_{t} u f \\
= & -\int u \partial_{x}^{3} u f-3 \int u \partial_{x}^{-1} \partial_{y}^{2} u f+6 \int u^{3} \partial_{x} u f-6 \int u \partial_{x} u \partial_{x}^{-1} \partial_{y} u f \\
= & \int \partial_{x} u \partial_{x}^{2} u f+\int u \partial_{x}^{2} u f^{\prime}+3 \int \partial_{y} u \partial_{x}^{-1} \partial_{y} u f \\
& -\frac{3}{2} \int u^{4} f^{\prime}+3 \int u^{2} \partial_{y} u+3 \int u^{2} \partial_{x}^{-1} \partial_{y} u f \\
= & -\frac{3}{2} \int\left(\partial_{x} u\right)^{2} f^{\prime}-\int u \partial_{x} u f^{\prime \prime}-\frac{3}{2} \int\left(\partial_{x}^{-1} \partial_{y} u\right)^{2} f^{\prime}-\frac{3}{2} \int u^{4} f^{\prime}+3 \int u^{2} \partial_{x}^{-1} \partial_{y} u f^{\prime} \\
= & -\frac{3}{2} \int\left\{\left(\partial_{x} u\right)^{2}+\left(\partial_{x}^{-1} \partial_{y} u-u^{2}\right)^{2}\right\} f^{\prime}+\frac{1}{2} \int u^{2} f^{\prime \prime \prime} .
\end{aligned}
$$

For solutions in $\mathcal{E}$ we use a standard density argument and the continuous dependence of the solution upon the initial data.

Proof of (74). It is direct from Bourgain's arguments that the IVP for the KP II equation is well-posed in $\mathcal{E}$ (see also Appendix in 2]). Such a solution can be approached by more regular solutions, so that the following calculations make sense:

$$
\begin{aligned}
\frac{1}{2} \frac{d}{d t} \int v^{2} f & =\int v \partial_{t} v f \\
& =-\int v \partial_{x}^{3} v f-3 \int v \partial_{x}^{-1} \partial_{y} v f-6 \int v^{2} \partial_{x} v f \\
& =-\frac{3}{2} \int\left(\partial_{x} v\right)^{2} f^{\prime}-\frac{3}{2} \int\left(\partial_{x}^{-1} \partial_{y} v\right)^{2} f^{\prime}+\frac{1}{2} \int v^{2} f^{\prime \prime \prime}+2 \int v^{3} f^{\prime}
\end{aligned}
$$

We are now ready to prove Proposition 9

Proof of Proposition 9. Let $u \in C(\mathbf{R}, \mathcal{E})$ be the solution of (11) associated to the initial data $u_{0} \in \mathcal{E}$. Let $v \in C\left(\mathbf{R}, L^{2}\right)$ be the corresponding solution of (9) associated to $u$ by the Miura transform

$$
v=\partial_{x} u+\partial_{x}^{-1} \partial_{y} u-u^{2}, \quad v_{0}=v(0) \in L^{2} .
$$

Let $\beta>0$ arbitrary and let $\sigma=\beta / 2$. We define

$$
\phi(x)=\frac{2}{\pi} \arctan \left(\exp \left(\frac{\sqrt{\sigma}}{2} x\right)\right),
$$

so that $\lim _{+\infty} \phi=1, \lim _{-\infty} \phi=0$, and $\forall x \in \mathbf{R}, \phi(-x)=1-\phi(x)$. Note that by direct calculations

$$
\phi^{\prime}(x)=\frac{\sqrt{\sigma}}{2 \pi \cosh \left(\frac{\sqrt{\sigma} x}{2}\right)}, \quad \phi^{\prime \prime \prime}(x) \leq \frac{\sigma}{4} \phi^{\prime}(x) .
$$


For $t_{0}>0$, we will use the following two quantities:

$$
\begin{aligned}
& I_{t_{0}}(t)=\int u^{2}(t, x, y) \phi\left(x-\sigma\left(t+t_{0}\right)\right) d x d y, \\
& J_{t_{0}}(t)=\int v^{2}(t, x, y) \phi\left(x-\sigma\left(t+t_{0}\right)\right) d x d y .
\end{aligned}
$$

Roughly speaking, $I_{t_{0}}(t)$ and $J_{t_{0}}(t)$ measure the quantity of the $L^{2}$ norm of $u(t)$, respectively $v(t)$ for $x>\sigma\left(t+t_{0}\right)$.

First, we use $I_{t_{0}}(t)$. Let $\tilde{x}=x-\sigma\left(t+t_{0}\right)$, so that $I_{t_{0}}(t)=\int u^{2}(t) \phi(\tilde{x})$. By Lemma [13, and then (75), we have

$$
\begin{aligned}
\frac{d}{d t} I_{t_{0}}(t) & =-3 \int\left\{\left(\partial_{x} u\right)^{2}+\left(\partial_{x}^{-1} \partial_{y} u-u^{2}\right)^{2}\right\} \phi^{\prime}(\tilde{x})+\int u^{2} \phi^{\prime \prime \prime}(\tilde{x})-\sigma \int u^{2} \phi^{\prime}(\tilde{x}) \\
& \leq-3 \int\left\{\left(\partial_{x} u\right)^{2}+\left(\partial_{x}^{-1} \partial_{y} u-u^{2}\right)^{2}\right\} \phi^{\prime}(\tilde{x})-\frac{3 \sigma}{4} \int u^{2} \phi^{\prime}(\tilde{x}) .
\end{aligned}
$$

Integrating this inequality between 0 and $t_{0}$, we obtain

$$
I_{t_{0}}\left(t_{0}\right)+3 \int_{0}^{t_{0}} \int\left\{\left(\partial_{x} u\right)^{2}+\left(\partial_{x}^{-1} \partial_{y} u-u^{2}\right)^{2}+\frac{\sigma}{4} u^{2}\right\} \phi^{\prime}(\tilde{x}) d t \leq I_{t_{0}}(0) .
$$

Since $\phi(x)$ is increasing, we have

$$
I_{t_{0}}(0)=\int u^{2}(0, x, y) \phi\left(x-\sigma t_{0}\right) d x d y \leq \phi\left(-\frac{\sigma t_{0}}{2}\right) \int u^{2}(0)+\int_{x>\frac{\sigma t_{0}}{2}} u^{2}(0, x, y) d x d y,
$$

and then

$$
\lim _{t_{0} \rightarrow+\infty} I_{t_{0}}(0)=0 .
$$

In particular, we get from (76)

$$
\lim _{t_{0} \rightarrow+\infty} I_{t_{0}}\left(t_{0}\right)=0, \quad \text { and so } \lim _{t_{0} \rightarrow+\infty} \int_{x>2 \sigma t_{0}=\beta t_{0}} u^{2}\left(t_{0}, x, y\right) d x d y=0 .
$$

But (76) contains more information: we also have

$$
\lim _{t_{0} \rightarrow+\infty} \int_{0}^{t_{0}} \int\left\{\left(\partial_{x} u\right)^{2}+\left(\partial_{x}^{-1} \partial_{y} u-u^{2}\right)^{2}+u^{2}\right\} \phi^{\prime}(\tilde{x}) d t=0 .
$$

We now use $J_{t_{0}}(t)$. We claim the following:

$$
J_{t_{0}}\left(t_{0}\right)-J_{t_{0}}(0) \leq C \int_{0}^{t_{0}} \int v^{2} \phi^{\prime}(\tilde{x}) d t .
$$

Note that in the previous inequality, all terms make sense for an $L^{2}$ solution. However, to prove (78), we will use quantities that are defined only for solutions in $\mathcal{E}$. Therefore, to justify (78) rigorously for all $L^{2}$ solutions, we would use a density argument. For the sake of simplicity, we make the calculations directly on $v(t)$, as if it were in $\mathcal{E}$. By Lemma 13 and (75), we have

$$
\begin{aligned}
\frac{d}{d t} J_{t_{0}}(t) & =-3 \int\left\{\left(\partial_{x} v\right)^{2}+\left(\partial_{x}^{-1} \partial_{y} v\right)^{2}-4 v^{3}\right\} \phi^{\prime}(\tilde{x})+\int v^{2} \phi^{\prime \prime \prime}(\tilde{x})-\sigma \int v^{2} \phi^{\prime}(\tilde{x}) \\
& \leq-3 \int\left\{\left(\partial_{x} v\right)^{2}+\left(\partial_{x}^{-1} \partial_{y} v\right)^{2}-4 v^{3}\right\} \phi^{\prime}(\tilde{x}) .
\end{aligned}
$$


To treat the nonlinear term $\int v^{3} \phi^{\prime}(\tilde{x})$ that has no sign in the previous inequality, we need the following Sobolev type inequality:

$$
\int v^{4}\left(\phi^{\prime}(\tilde{x})\right)^{2} \leq C\left(\int v^{2} \phi^{\prime}(\tilde{x})\right)^{\frac{1}{2}}\left(\int\left\{\left(\partial_{x} v\right)^{2}+\left(\partial_{x}^{-1} \partial_{y} v\right)^{2}+v^{2}\right\} \phi^{\prime}(\tilde{x})\right)^{\frac{3}{2}},
$$

which is a direct consequence of Lemma 7, and arguments in 2], Proof of Claim 1. Then, for $\varepsilon>0$,

$$
\begin{aligned}
& \left|\int v^{3} \phi^{\prime}(\tilde{x})\right| \leq\left(\int v^{4}\left(\phi^{\prime}(\tilde{x})\right)^{2}\right)^{\frac{1}{2}}\left(\int v^{2}\right)^{\frac{1}{2}} \\
& \quad \leq C\left(\int v^{2} \phi^{\prime}(\tilde{x})\right)^{\frac{1}{4}}\left(\int\left\{\left(\partial_{x} v\right)^{2}+\left(\partial_{x}^{-1} \partial_{y} v\right)^{2}+v^{2}\right\} \phi^{\prime}(\tilde{x})\right)^{\frac{3}{4}}\left(\int v^{2}\right)^{\frac{1}{2}} \\
& \quad \leq C \varepsilon^{\frac{4}{3}}\left(\int v^{2}\right)^{\frac{2}{3}}\left(\int\left\{\left(\partial_{x} v\right)^{2}+\left(\partial_{x}^{-1} \partial_{y} v\right)^{2}+v^{2}\right\} \phi^{\prime}(\tilde{x})\right)+\frac{1}{\varepsilon^{4}} \int v^{2} \phi^{\prime}(\tilde{x}) .
\end{aligned}
$$

Therefore, since $\int v^{2}$ is constant in time, choosing $\varepsilon>0$ small enough, we obtain

$$
\frac{d}{d t} J_{t_{0}}(t) \leq K \int v^{2} \phi^{\prime}(\tilde{x})
$$

Integrating between 0 and $t_{0}$, we obtain (78).

Since $\int v^{2} \phi^{\prime}(\tilde{x}) \leq 2 \int\left\{\left(\partial_{x} u\right)^{2}+\left(\partial_{x}^{-1} \partial_{y} u-u^{2}\right)^{2}\right\} \phi^{\prime}(\tilde{x})$, we obtain

$$
J_{t_{0}}(t) \leq J_{t_{0}}(0)+2 K \int_{0}^{t_{0}} \int\left\{\left(\partial_{x} u\right)^{2}+\left(\partial_{x}^{-1} \partial_{y} u-u^{2}\right)^{2}\right\} \phi^{\prime}(\tilde{x}) .
$$

From $\lim _{t_{0} \rightarrow+\infty} J_{t_{0}}(0)=0$ and (77), we conclude

$$
\lim _{t_{0} \rightarrow+\infty} J_{t_{0}}\left(t_{0}\right)=0
$$

Finally, we have

$$
\begin{aligned}
J_{t_{0}}\left(t_{0}\right)= & \int\left(\partial_{x} u+\partial_{x}^{-1} \partial_{y} u-u^{2}\right)^{2} \phi(\tilde{x}) \\
= & \int\left\{\left(\partial_{x} u\right)^{2}+\left(\partial_{x}^{-1} \partial_{y} u-u^{2}\right)^{2}\right\} \phi(\tilde{x}) \\
& +2 \int\left(\partial_{x} u \partial_{x}^{-1} \partial_{y} u\right) \phi(\tilde{x})-2 \int u^{2} \partial_{x} u \phi(\tilde{x}) .
\end{aligned}
$$

On one hand,

$$
\int\left(\partial_{x} u \partial_{x}^{-1} \partial_{y} u\right) \phi(\tilde{x})=-\int u \partial_{y} u \phi(\tilde{x})-\int u \partial_{x}^{-1} \partial_{y} u \phi^{\prime}(\tilde{x})=-\int u \partial_{x}^{-1} \partial_{y} u \phi^{\prime}(\tilde{x}),
$$

and since

$$
\left|\int u \partial_{x}^{-1} \partial_{y} u \phi^{\prime}(\tilde{x})\right| \leq\left(\int u^{2} \phi^{\prime}(\tilde{x})\right)^{\frac{1}{2}}\left(\int\left(\partial_{x}^{-1} \partial_{y} u\right)^{2} \phi^{\prime}(\tilde{x})\right)^{\frac{1}{2}} \leq C\left(\int u^{2} \phi(\tilde{x})\right)^{\frac{1}{2}}
$$

(we have used $\phi^{\prime} \leq C \phi$ ), this term converges to 0 as $t_{0} \rightarrow+\infty$. On the other hand,

$$
\int \partial_{x} u u^{2} \phi(\tilde{x})=-\frac{1}{3} \int u^{3} \phi^{\prime}(\tilde{x})
$$


and since

$$
\left|\int u^{3} \phi^{\prime}(\tilde{x})\right| \leq\left(\int u^{2} \phi^{\prime}(\tilde{x})\right)^{\frac{1}{2}}\left(\int u^{4} \phi^{\prime}(\tilde{x})\right)^{\frac{1}{2}} \leq C\left(\int u^{2} \phi(\tilde{x})\right)^{\frac{1}{2}},
$$

this term also converges to 0 .

Finally, since $\left(\partial_{x}^{-1} \partial_{y} u\right)^{2} \leq 2\left(\partial_{x}^{-1} \partial_{x} u-u^{2}\right)^{2}+2 u^{4}$, and since $\lim _{t_{0} \rightarrow+\infty} \int u^{4} \phi(\tilde{x})=$ 0 as before, we obtain

$$
\lim _{t_{0} \rightarrow+\infty} \int\left\{\left(\partial_{x} u\left(t_{0}\right)\right)^{2}+\left(\partial_{x}^{-1} \partial_{y} u\left(t_{0}\right)\right)^{2}\right\} \phi(\tilde{x})=0,
$$

which proves (172).

\section{Appendix A}

In this Appendix, we prove the following property:

Lemma 14. (i) If $u(t, x, y)$ is a solution of

$$
\partial_{t} u+\partial_{x}^{3} u+3 \partial_{x}^{-1} \partial_{y}^{2} u-6 u^{2} \partial_{x} u+6 \partial_{x} u \partial_{x}^{-1} \partial_{y} u=0,
$$

then

$$
v(t, x, y)=\partial_{x} u(t, x, y)+\partial_{x}^{-1} \partial_{y} u(t, x, y)-u^{2}(t, x, y)
$$

and

$$
\tilde{v}(t, x, y)=-\partial_{x} u(t, x, y)+\partial_{x}^{-1} \partial_{y} u(t, x, y)-u^{2}(t, x, y)
$$

are both solutions of

$$
\partial_{x}\left(\partial_{t} v+\partial_{x}^{3} v+6 v \partial_{x} v\right)+3 \partial_{y}^{2} v=0
$$

(ii) If $u(t, x, y)$ is a solution of

$$
\partial_{t} u+a \partial_{x}^{4} u+b \partial_{y}^{4} u+\partial_{x}^{3} u+3 \partial_{x}^{-1} \partial_{y}^{2} u-6 u^{2} \partial_{x} u+6 \partial_{x} u \partial_{x}^{-1} \partial_{y} u=0,
$$

then $v(t, x, y)=\partial_{x} u(t, x, y)+\partial_{x}^{-1} \partial_{y} u(t, x, y)-u^{2}(t, x, y)$ is a solution of

$$
\begin{aligned}
& \partial_{x}\left(\partial_{t} v+a \partial_{x}^{4} v+b \partial_{y}^{4} v+\partial_{x}^{3} v+6 v \partial_{x} v\right)+3 \partial_{y}^{2} v \\
& \quad=-4 \partial_{x}\left[a\left(\partial_{x}^{2}\left(\partial_{x} u\right)^{2}-\frac{1}{2}\left(\partial_{x}^{2} u\right)^{2}\right)+b\left(\partial_{y}^{2}\left(\partial_{y} u\right)^{2}-\frac{1}{2}\left(\partial_{y}^{2} u\right)^{2}\right)\right] .
\end{aligned}
$$

Proof of (i). We set $w=\partial_{x}^{-1} \partial_{y} u-u^{2}$, so that $\partial_{x} w=\partial_{y} u-2 u \partial_{x} u$. Then, the equation of $u$ can be written as follows:

$$
\partial_{t} u+\partial_{x}^{3} u+3 \partial_{x}^{-1} \partial_{y}^{2} u=-6 w \partial_{x} u
$$

Since $-6 w \partial_{x} u=-6 \partial_{x}(w u)+6 u \partial_{x} w=-6 \partial_{x}(w u)-4 \partial_{x}\left(u^{3}\right)+3 \partial_{y}\left(u^{2}\right)$, we also have

$$
\partial_{t} u+\partial_{x}^{3} u+3 \partial_{x}^{-1} \partial_{y}^{2} u=-6 \partial_{x}(w u)-4 \partial_{x}\left(u^{3}\right)+3 \partial_{y}\left(u^{2}\right) .
$$

By $\partial_{x}^{-1} \partial_{y}^{2} u=\partial_{y} w+\partial_{y}\left(u^{2}\right)$, we also have the following form of equation (80):

$$
\partial_{t} u+\partial_{x}^{3} u=-6 w \partial_{x} u-3 \partial_{y}\left(u^{2}\right)-3 \partial_{y} w .
$$

Both formulations (83) and (84) will be useful in what follows.

First, we claim the following equation for $w(t, x, y)$ :

$$
\partial_{t}\left(\partial_{x} w\right)+\partial_{x}^{3}\left(\partial_{x} w\right)+3 \partial_{x}^{-1} \partial_{y}^{2}\left(\partial_{x} w\right)=-3 \partial_{x}^{2}\left(w^{2}+\left(\partial_{x} u\right)^{2}\right) .
$$


Proof of 855). Since $\partial_{x} w=\partial_{y} u-\partial_{x}\left(u^{2}\right)$, we have

$\partial_{t}\left(\partial_{x} w\right)+\partial_{x}^{3}\left(\partial_{x} w\right)+3 \partial_{x}^{-1} \partial_{y}^{2}\left(\partial_{x} w\right)=\partial_{y}\left[\partial_{t} u+\partial_{x}^{3} u+3 \partial_{x}^{-1} \partial_{y}^{2} u\right]$

$$
-\partial_{x}\left[2 u\left(\partial_{t} u+\partial_{x}^{3} u\right)+3 \partial_{x}\left(\partial_{x} u\right)^{2}\right]-3 \partial_{y}^{2}\left(u^{2}\right) .
$$

Therefore, using (83) and (84), we obtain

$$
\begin{gathered}
\partial_{t}\left(\partial_{x} w\right)+\partial_{x}^{3}\left(\partial_{x} w\right)+3 \partial_{x}^{-1} \partial_{y}^{2}\left(\partial_{x} w\right)=\partial_{x} \partial_{y}\left[-6 u w-4 u^{3}\right]+3 \partial_{y}^{2}\left(u^{2}\right) \\
+2 \partial_{x}\left[6 u w \partial_{x} u+3 u \partial_{y}\left(u^{2}\right)+3 u \partial_{y} w\right]-3 \partial_{x}^{2}\left(\partial_{x} u\right)^{2}-3 \partial_{y}^{2}\left(u^{2}\right) \\
=-6 \partial_{x}\left[\left(\partial_{y} u-2 u \partial_{x} u\right) w\right]-3 \partial_{x}^{2}\left(\partial_{x} u\right)^{2}=-3 \partial_{x}^{2}\left(w^{2}+\left(\partial_{x} u\right)^{2}\right) .
\end{gathered}
$$

Thus (85) is proved.

Since

$$
\partial_{t}\left(\partial_{x}^{2} u\right)+\partial_{x}^{3}\left(\partial_{x}^{2} u\right)+3 \partial_{x}^{-1} \partial_{y}^{2}\left(\partial_{x}^{2} u\right)=-6 \partial_{x}^{2}\left(w \partial_{x} u\right),
$$

we have for $v=\partial_{x} u+w$,

$$
\partial_{t}\left(\partial_{x} v\right)+\partial_{x}^{3}\left(\partial_{x} v\right)+3 \partial_{x}^{-1} \partial_{y}^{2}\left(\partial_{x} v\right)=-3 \partial_{x}^{2}\left(\left(w+\partial_{x} u\right)^{2}\right)=-3 \partial_{x}^{2}\left(v^{2}\right),
$$

so that $v$ satisfies (80). We check that $\tilde{v}=-\partial_{x} u+w$ is also a solution.

The proof of (ii) is very similar, however because of the parabolic terms, there are still terms depending on $u$ in the equation of $v$. In this case, (86) becomes

$$
\begin{aligned}
& \partial_{t}\left(\partial_{x} w\right)+a \partial_{x}^{4}\left(\partial_{x} w\right)+b \partial_{y}^{4}\left(\partial_{x} w\right)+\partial_{x}^{3}\left(\partial_{x} w\right)+3 \partial_{x}^{-1} \partial_{y}^{2}\left(\partial_{x} w\right) \\
& \quad=\partial_{y}\left[\partial_{t} u+a \partial_{x}^{4} u+b \partial_{y}^{4} u+\partial_{x}^{3} u+3 \partial_{x}^{-1} \partial_{y}^{2} u\right] \\
& \quad-\partial_{x}\left[2 u\left(\partial_{t} u+a \partial_{x}^{4} u+b \partial_{y}^{4} u+\partial_{x}^{3} u\right)+a F+b G+3 \partial_{x}\left(\partial_{x} u\right)^{2}\right]-3 \partial_{y}^{2}\left(u^{2}\right),
\end{aligned}
$$

where $F=\partial_{x}^{4}\left(u^{2}\right)-2 u \partial_{x}^{4} u$ and $G=2 \partial_{y}^{4}\left(u^{2}\right)-2 u \partial_{y}^{4} u$. We have

$$
F=2\left[4 \partial_{x}^{3} u \partial_{x} u+3\left(\partial_{x}^{2} u\right)^{2}\right]=4\left[\partial_{x}^{2}\left(\partial_{x} u\right)^{2}-\frac{1}{2}\left(\partial_{x}^{2} u\right)^{2}\right] .
$$

Similarly, $G=4\left[\partial_{y}^{2}\left(\partial_{y} u\right)^{2}-\frac{1}{2}\left(\partial_{y}^{2} u\right)^{2}\right]$. We finish the proof of (ii) as for (i).

\section{Appendix B. Proof of Theorem 2}

We repeat the argument of Theorem 1.II in [26]. Let $V \in L^{2} \cap L^{1}$ be such that $\|V\|_{L^{2}}+\|V\|_{L^{1}} \leq \alpha_{0}$.

First, we prove the existence of $W \in L^{\infty}$ such that

$$
\partial_{x}^{2} W+\partial_{y} W=-V(1+W)
$$

by a contraction argument. Note that taking the Fourier transform of (89), it is sufficient to find $\hat{W}=F \in L^{1}$ such that

$$
\left(-\xi^{2}+i \mu\right) F=-\left(\hat{V}+\frac{1}{(2 \pi)^{2}} \hat{V} \star F\right) .
$$

Thus, we set, for $F_{1} \in L^{1}$,

$$
\Psi: F_{1} \mapsto-f(\xi, \mu)\left(\hat{V}+\frac{1}{(2 \pi)^{2}} \hat{V} \star F\right),
$$


where $f(\xi, \mu)=\frac{1}{-\xi^{2}+i \mu}$. We have $f=f_{1}+f_{2} \in L^{1}+L^{2}$ (indeed, $\int_{|\mu|<1}|f|<\infty$ and $\left.\int_{|\mu|>1}|f|^{2}<\infty\right)$. Thus, by Holder and Young inequalities,

$$
\begin{aligned}
\left\|\Psi\left(F_{1}\right)\right\|_{L^{1}} & \leq\left\|f_{1}\right\|_{L^{1}}\left[\|\hat{V}\|_{L^{\infty}}+\left\|\hat{V} \star F_{1}\right\|_{L^{\infty}}\right]+\left\|f_{2}\right\|_{L^{2}}\left[\|\hat{V}\|_{L^{2}}+\left\|\hat{V} \star F_{1}\right\|_{L^{2}}\right] \\
& \leq C\left(1+\left\|F_{1}\right\|_{L^{1}}\right)\left[\|\hat{V}\|_{L^{\infty}}+\|\hat{V}\|_{L^{2}}\right] \\
& \leq C\left(1+\left\|F_{1}\right\|_{L^{1}}\right)\left[\|V\|_{L^{1}}+\|V\|_{L^{2}}\right] .
\end{aligned}
$$

Moreover,

$$
\left\|\Psi\left(F_{1}\right)-\Psi\left(F_{2}\right)\right\|_{L^{1}} \leq C\left\|F_{1}-F_{2}\right\|_{L^{1}}\left[\|\hat{V}\|_{L^{\infty}}+\|\hat{V}\|_{L^{2}}\right] .
$$

Therefore, if $\alpha_{0}$ is small enough, $\Psi$ is a contraction in $\mathcal{B}_{1 / 2}=\left\{F \in L^{1}:\|F\|_{L^{1}} \leq \frac{1}{2}\right\}$. Thus, there exists $F \in L^{1}$, with $\|F\|_{L^{1}} \leq \frac{1}{2}$, such that (90) is satisfied. Define $W \in L^{\infty}$ such that $\hat{W}=F$; then $W$ satisfies (89) and $\|W\|_{L^{\infty}} \leq \frac{1}{(2 \pi)^{2}}\|F\|_{L^{1}} \leq \frac{1}{8 \pi^{2}}$. Since $V$ is real-valued, it is clear that $W$ is also real-valued.

Now, we obtain more properties on $W$. Since $\hat{W} \in L^{1}$ and $\hat{V} \in L^{2} \cap L^{\infty}$, we have from (90)

$$
\left|\xi^{2}+i \mu\right||\hat{W}|=\left(\xi^{4}+\mu^{2}\right)^{\frac{1}{2}}|\hat{W}| \in L^{2} \cap L^{\infty} .
$$

Thus $|-\xi|^{2} \hat{W} \in L^{2} \cap L^{\infty}$ and $|\mu| \hat{W} \in L^{2} \cap L^{\infty}$, which first implies $\partial_{x}^{2} W, \partial_{y} W \in L^{2}$. Moreover,

$$
\int \xi^{2}|\hat{W}|^{2} \leq\left\|\xi^{2} \hat{W}\right\|_{L^{\infty}} \int|\hat{W}|<\infty
$$

and so $\partial_{x} W \in L^{2}$ (by Lemma 7 , we also have $\partial_{x} W \in L^{4}$ ).

We set $Z=\ln (1+W)$, since $|W| \leq \frac{1}{8 \pi^{2}}, Z$ is well defined and in $L^{\infty}$. Moreover,

$$
\partial_{x} Z=\frac{\partial_{x} W}{1+W}, \quad \partial_{y} Z=\frac{\partial_{y} W}{1+W}, \quad \partial_{x}^{2} Z=\frac{\partial_{x}^{2} W}{1+W}-\left(\frac{\partial_{x} W}{1+W}\right)^{2},
$$

and so $\partial_{x} Z \in L^{2}, \partial_{y} Z \in L^{2}, \partial_{x}^{2} Z \in L^{2}$ and $\partial_{x}^{2} Z+\left(\partial_{x} Z\right)^{2}=\frac{\partial_{x}^{2} W}{1+W}$. Thus by (899), we obtain the following equation for $Z$ :

$$
\partial_{x}^{2} Z+\partial_{y} Z+\left(\partial_{x} Z\right)^{2}=-V
$$

Finally, we set $U=-\partial_{x} Z$, so that $U \in \mathcal{E}$ and

$$
\partial_{x} U+\partial_{x}^{-1} \partial_{y} U-U^{2}=V .
$$

\section{Appendix C. Proof of some nonisotropic Sobolev inequalities}

In this Appendix, we prove the following inequalities, for $2<q<6$ (the case $q=2$ is trivial and $q=6$ is an endpoint case that we do not consider here; see [1]):

$$
\|\psi\|_{L^{q}}^{q} \leq K\|\psi\|_{L^{2}}^{\frac{6-q}{2}}\left\|\partial_{x} \psi\right\|_{L^{2}}^{q-2}\left\|\partial_{x}^{-1} \partial_{y} \psi\right\|_{L^{2}}^{\frac{q-2}{2}} .
$$

Let $\frac{1}{q}+\frac{1}{q^{\prime}}=1$. We have $\frac{6}{5}<q^{\prime}<2$ and $\|\psi\|_{L^{q}} \leq C\|\hat{\psi}\|_{L^{q^{\prime}}}$. But,

$$
|\hat{\psi}(\xi, \eta)|^{q^{\prime}} \leq\left(1+|\xi|^{2}+\left|\frac{\eta}{\xi}\right|^{2}\right)^{-\frac{q^{\prime}}{2}}\left[\left(1+|\xi|^{2}+\left|\frac{\eta}{\xi}\right|^{2}\right)^{\frac{1}{2}}|\hat{\psi}(\xi, \eta)|\right]^{q^{\prime}} .
$$


For $2<q<6$, we claim $\int\left(1+|\xi|^{2}+\left|\frac{\eta}{\xi}\right|^{2}\right)^{-\frac{q^{\prime}}{2-q^{\prime}}} d \xi d \eta<+\infty$. Indeed, considering the region $\xi>0, \eta>0$, and changing the variable $\eta^{\prime}=\frac{\eta}{\xi\left(1+\xi^{2}\right)^{\frac{1}{2}}}$, we have

$$
\int_{\xi>0, \eta>0}\left(1+|\xi|^{2}+\left|\frac{\eta}{\xi}\right|^{2}\right)^{-\frac{q^{\prime}}{2-q^{\prime}}} d \xi d \eta=\int_{\xi>0, \eta^{\prime}>0} \frac{\xi\left(1+\xi^{2}\right)^{\frac{1}{2}-\frac{q^{\prime}}{2-q^{\prime}}}}{\left(1+\eta^{\prime 2}\right)^{\frac{q^{\prime}}{2-q^{\prime}}}} d \xi d \eta^{\prime} .
$$

Since $\frac{q^{\prime}}{2-q^{\prime}}>\frac{3}{2}$, the integral in $\eta^{\prime}$ is finite, and since the power of $\xi$ at $+\infty$ is $2-\frac{2 q^{\prime}}{2-q^{\prime}}<-1$, the integral in $\xi$ is also finite ( $q=6$ is critical), which proves the claim.

Thus, by Holder inequality, it follows from (94) that

$$
\|\psi\|_{L^{q}} \leq K\left(\|\psi\|_{L^{2}}+\left\|\partial_{x} \psi\right\|_{L^{2}}+\left\|\partial_{x}^{-1} \partial_{y} \psi\right\|_{L^{2}}\right) .
$$

The multiplicative form (93) is easily obtained by scaling arguments.

\section{REFERENCES}

[1] O. V. Besov, V. P. Il'in and S. M. Nikolskii, Integral representations of functions and imbedding theorems, vol. I, J. Wiley, 1978. MR0519341 (80f:46030a)

[2] A. de Bouard and Y. Martel, Non existence of $L^{2}$-compact solutions of the KadomtsevPetviashvili II equation, Math. Ann. 328, (2004) 525-544. MR2036335 (2004m:35227)

[3] A. de Bouard and J.-C. Saut, Solitary waves of generalized Kadomtsev-Petviashvili equations, Ann. Inst. H. Poincaré, Anal. Non Lin. 14 (1997), 211-236. MR.1441393 (98a:35115)

[4] J. Bourgain, On the Cauchy problem for the Kadomtsev-Petviashvili equation, Geom. Funct. Anal. 3, (1993) 315-341. MR1223434 (94d:35142)

[5] R.R. Coifman and Y. Meyer, Au delà des opérateurs pseudodifférentiels, Astérisque 57, (1978). MR0518170 (81b:47061)

[6] R.J. Iório Jr. and W.V. L. Nunes, On equations of KP-type, Proc. Royal Soc. Edinburg 128A, (1998) 725-743. MR.1635416 (99j:35189)

[7] M. Jimbo and T. Miura, Solitons and infinite dimensional Lie algebras, Publ. Res. Int. Math. Sci. 19, (1983) 943-1001. MR0723457 (85i:58060)

[8] T. Kato, On the Cauchy problem for the (generalized) Korteweg-de Vries equation, Advances in MAthematics Supplementary Studies, Studies in Applied Math. 8, (1983) 93-128. MR0759907 (86f:35160)

[9] T. Kato and G. Ponce, Commutator estimates and the Euler and Navier-Stokes equations, Comm. Pure Appl. Math. 41, (1998) 891-907. MR0951744 (90f:35162)

[10] C.E. Kenig, G. Ponce and L. Vega, Well-posedness of the initial value problem for the Korteweg-de Vries equation, J. Amer. Math. Soc. 4, (1991) 323-347. MR1086966 (92c:35106)

[11] C.E. Kenig, G. Ponce and L. Vega, Well-posedness and scattering results for the generalized Korteweg-de Vries equation via the contraction principle, Comm. Pure Appl. Math. 46, (1993) 527-620. MR1211741 (94h:35229)

[12] C.E. Kenig and S. Ziesler, Maximal function estimates with applications to a modified Kadomstev-Petviashvili equation, Commun. Pure Appl. Anal. 4, (2005) 45-91. MR2126278 $(2005 \mathrm{j}: 35197)$

[13] C.E. Kenig and S. Ziesler, Local well posedness for modified Kadomstev-Petviashvili equations, Diff. Int. Eq. 18, (2005) 1111-1146. MR2162626

[14] B.G. Konopelchenko, On the Gauge-invariant description of the evolution equations integrable by Gelfand-Dikij spectral problems, Phys. Lett. A 92, (1982) 323-327. MR0687494 (84e:58027)

[15] B.G. Konopelchenko and V.G. Dubrovsky, On the interrelation between the solutions of the mKP and KP equations via the Miura transformation, J. Phys. A : Math. Gen. 24, (1991) 4315-4324. MR1127296 (92i:35108)

[16] B.G. Konopelchenko and V.G. Dubrovsky, Inverse spectral transform for the modified Kadomtsev-Petviashvili equation, Stud. Appl. Math. 86, (1992) 219-268. MR 1150011 (92k:35244) 
[17] J.L. Lions, Quelques méthodes de résolution des problèmes aux limites nonlinéaires, Dunod; Gauthier-Villars, Paris 1969. MR0259693 (41:4326)

[18] Y. Martel and F. Merle, A Liouville theorem for the critical generalized Korteweg-de Vries equation, J. Math. Pures Appl. 79, (2000) 339-425. MR1753061(2001i:37102)

[19] P. Isaza and J. Mejía, Local and global Cauchy problems for the Kadomtsev-Petviashvili (KP-II) equation in Sobolev spaces of negative indices, Commun. Partial Differential Equations 26, (2001) 1027-1054. MR1843294 (2002e:35205)

[20] R.M. Miura, The Korteweg-de Vries equation: a survey of results, SIAM Review 18, (1976) 412-459. MR0404890 (53:8689)

[21] L. Molinet, On the asymptotic behavior of solutions to the (generalized) KadomtsevPetviashvili-Burgers equations, J. Diff. Eq. 152, (1999) 30-74. MR1672012 (99m:35212)

[22] L. Molinet and F. Ribaud, Well-posedness results for the generalized Benjamin-Ono equation with small initial data, J. Math. Pures Appl. 83, (2004) 277-311. MR2038121 (2005a:35252)

[23] J.-C. Saut, Remarks on the generalized Kadomtsev-Petviashvili equations, Indiana Univ. Math. J. 42, (1993) 1011-1026. MR1254130 (95j:35200)

[24] H. Takaoka and N. Tzvetkov, On the local regularity of the Kadomtsev-Petviashvili-II equation, Internat. Math. Res. Notices 2 (2001), 77-114. MR1810481(2001j:35243)

[25] S. Ukai, Local solutions of the Kadomtsev-Petviashvili equation, J. Fac. Sci. Univ. Tokyo, Sect. IA Math. 36, (1989) 193-209. MR1014996 (90i:35270)

[26] M.V. Wickerhauser, Inverse scattering for the heat operator and evolutions in $2+1$ variables, Commun. Math. Phys. 108, (1987) 67-89. MR0872141 (87m:35224)

Institute for Advanced Study and Department of Mathematics, University of ChiCAgo, Chicago, Illinois 60637

Centre de Mathématiques, Ecole Polytechnique 91128 Palaiseau Cedex, France

Current address: Department of Mathematics, Université de Versailles-Saint-Quentin-enYvelines, 78035 Versailles, France 